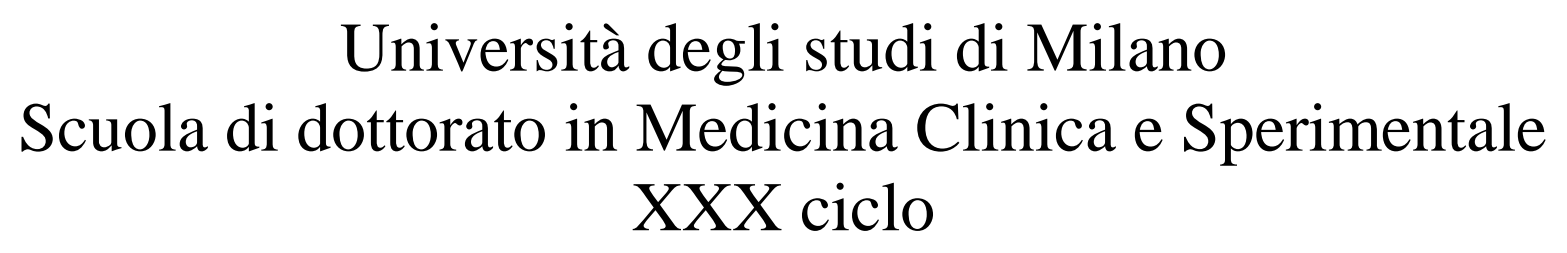

\title{
Down-regulation of hepatic MBOAT7 by hyperinsulinemia favors steatosis development
}

Tutor: Prof. Luca Valenti

PhD student: Dr. Marica Meroni Matricola: R10915-R19 


\section{Index}

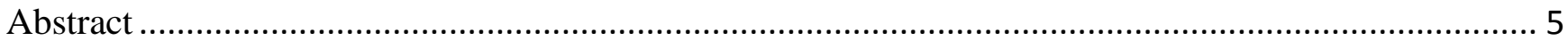

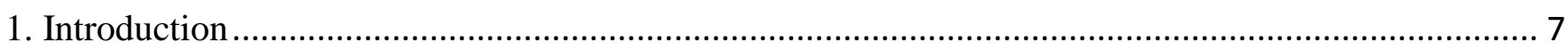

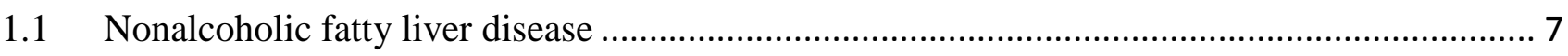

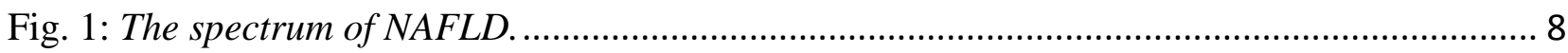

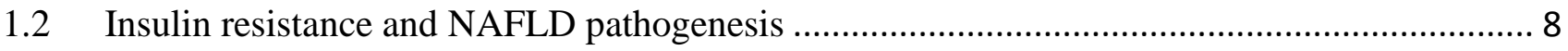

Fig. 2: The insulin signaling pathway in hepatocytes............................................................ 9

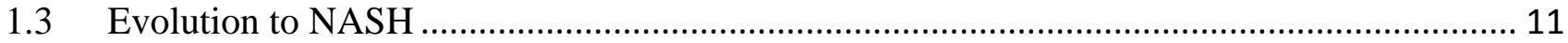

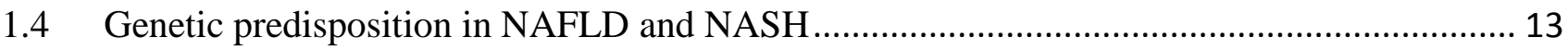

1.5 The MBOAT7 rs641738 variant is novel a risk factor for NAFLD onset and progression...... 15

Fig. 3: The rs641738 variant is associated with increased intrahepatic TG content in the DHS cohort and with the severity of histologic damage in the Liver Biopsy Cross-Sectional Cohort... 17

1.6 MBOAT7 function and pathogenic effect of the rs641738 variant.................................... 17

Fig. 4: Integrated model for the Land's cycle of phospholipids remodeling of the membranes and arachidonic acid mobilization. .............................................................................................. 20

Fig. 5: The rs641738 variant decreases MBOAT7 mRNA expression and synthesis level in liver biopsies of obese patients............................................................................................ 22

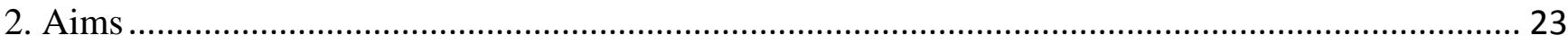

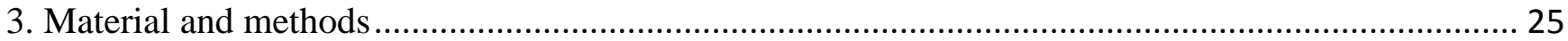

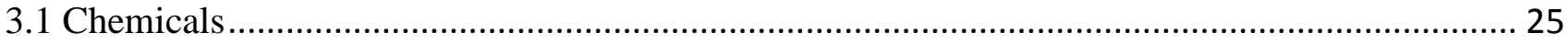

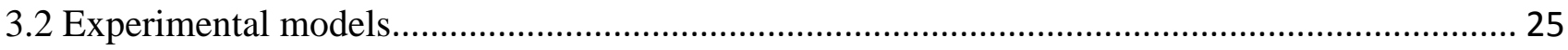

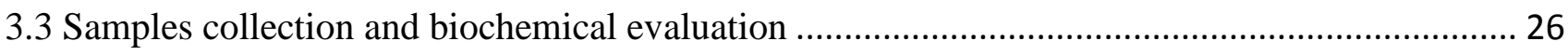

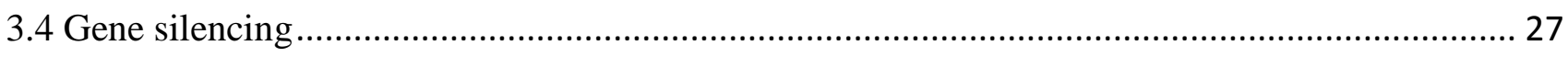

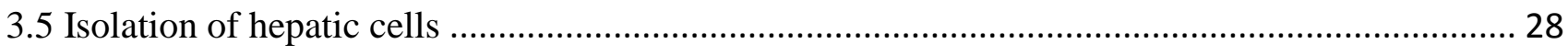

3.6 Isolation of leukocytes from peripheral blood ............................................................. 29

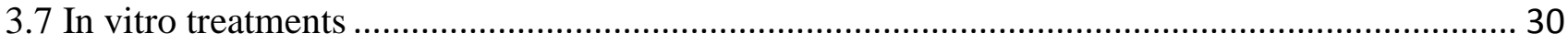

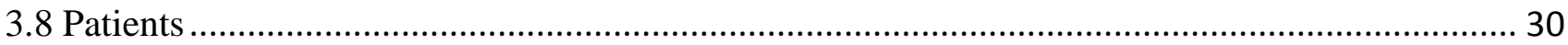

Table 1. Clinical characteristics of 119 severely obese patients (Bariatric surgery cohort), of whom MBOAT7 hepatic and adipose tissue expression was evaluated.............................................. 31

Table 2. Clinical characteristics of 72 NAFLD patients (Hepatology service cohort), of whom serum FFAs concentration was evaluated........................................................................ 32

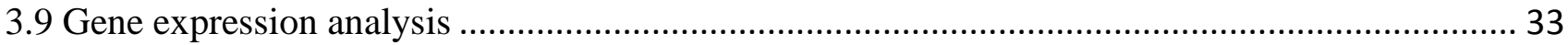

Table 3: Quantitative PCR analysis primer sequences ...................................................... 33

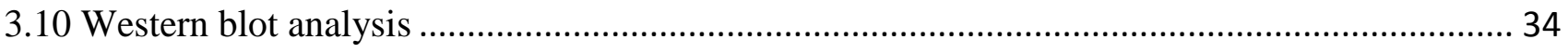


Table 4. Antibodies and dilution used in Western blotting and immunohistochemistry. 35

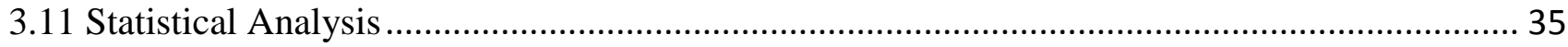

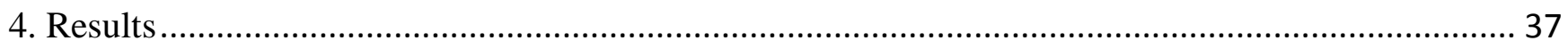

4.1 MBOAT7 down-regulation is associated with NAFLD severity independently of rs641737 C>T genotype

Table 5: Variables associated with hepatic MBOAT7 gene expression at multivariate generalized linear model analysis, adjusted for all the clinical features reported in the table, in 119 severely obese patients who underwent to liver biopsy during bariatric surgery. .................................. 38

Fig. 6: Hepatic MBOAT7 expression decreases with liver damage severity. ............................... 39

Table 6: Variables associated with adipose MBOAT7 gene expression at multivariate generalized linear model analysis in 119 severely obese patients who underwent to adipose tissue biopsy

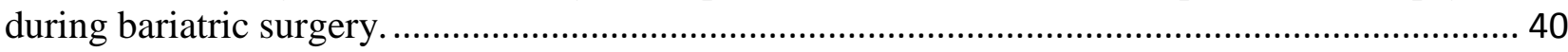

Fig. 7: MBOAT7 rs641738 C>T variant affects MBOAT7 expression in hepatocytes, but not in

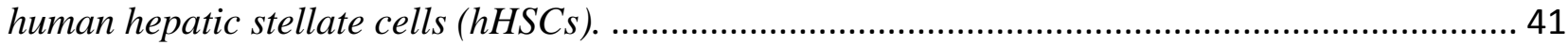

Fig. 8: MBOAT7 expression decreases with inflammatory cells activation ................................ 42

4.2 MBOAT7 expression is dampened in Lep ${ }^{o b / o b}$ mice ................................................................ 42

Fig. 9: MBOAT7 expression decreases in experimental model of NASH. ................................ 43

4.3 InsR+/- mice show blunted MBOAT7 expression, independently of diet................................ 44

Fig. 10: Insulin receptor haploinsufficient (InsR+/-) mice show decreased MBOAT7 expression,

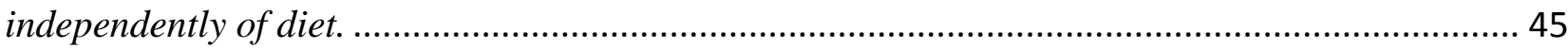

Fig. 11: Fructose supplementation to MCD does not exacerbate MBOAT7 down-regulation in

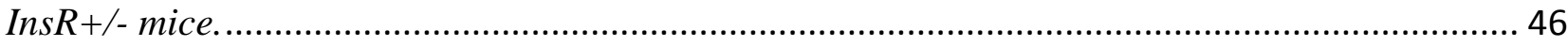

4.4 Hepatic MBOAT7 expression depends on nutritional state.............................................. 46

Table 7. Biochemical features of wild-type male mice fasted for 16 hours or refed (15 min, 1 hour,

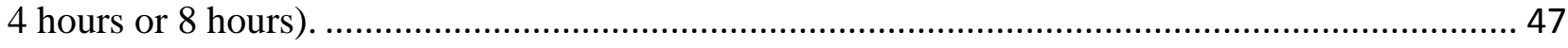

Fig. 12: Hepatic MBOAT7 expression depends on nutritional state .......................................... 48

Fig. 13: MBOAT5 increases in insulin resistant mice ........................................................... 49

4.5 MBOAT7 down-regulation is dependent on insulin signaling activation in primary hepatocytes50

Fig. 14: MBOAT7 down-regulation is dependent on insulin signaling activation in primary

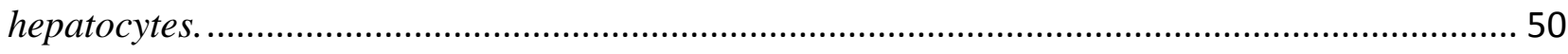

Fig. 15: mTORC1 inhibition does not affect the insulin-dependent MBOAT7 down-regulation.... 51 Fig. 16: In InsR+/- hepatocytes, the insulin-mediated suppression of Mboat7 expression is lost. 52 4.6 MBOAT7 down-regulation by insulin in primary mouse hepatocytes requires FOXO1 ............ 52

Fig. 17: FoxO1 and FoxA2 are predicted to bind to MBOAT7 promoter. ................................. 53

Fig. 18: MBOAT7 down-regulation by insulin in primary mouse hepatocytes requires FOXO1. . 54 
Fig. 19: InsR+/- treated with FoxO1-MPO re-stabilize MBOAT7 mRNA levels comparable to that observed in wild-type.

4.7 Hepatic MBOAT7 silencing induces microvesicular steatosis in vivo.................................... 55

Table 8. Biochemical features of wild-type mice, treated with Scramble and MPO MBOAT7. ... 56

Fig. 20: Hepatic MBOAT7 silencing induces microvesicular steatosis in vivo. 57

Fig. 21: Steatosis development in MBOAT7-MPO mice is apparently explained by enhanced expression of fatty acids transporters.

Fig. 22: No alteration in inflammation or in genes related to MBOAT7 is identified in MBOAT7MPO mice. 59

Fig. 23: MBOAT7 is silenced also in adipose tissue of MBOAT7-MPO mice, but not in brain..... 60 Table 9: Variables associated with FFAs dosage $(\mathrm{nmol} / \mu \mathrm{L})$ at multivariate generalized linear model analysis in 72 NAFLD patients (Hepatology cohort)

5. Discussion

6. Conclusion 69

7. References 


\section{Abstract}

Background and aims: Nonalcoholic fatty liver disease (NAFLD) is the most common chronic liver diseases in Western Countries. NAFLD could evolve into nonalcoholic steatohepatitis (NASH), which is associated with activation of fibrogenesis, possibly leading to cirrhosis and hepatocellular carcinoma (HCC). The genetic background plays a pivotal role in predisposing to development and progression of liver disease. Recently, our group have shown that the rs641738 C>T variant, in the Membrane bound o-acyltransferase domain-containing 7 locus (MBOAT7), increases the risk of NAFLD, NASH and fibrosis, by decreasing the hepatic expression of MBOAT7, a protein involved in phosphatidylinositol acyl-chain remodeling. Aim of this study was to evaluate the regulation of hepatic MBOAT7 and the impact on hepatic fat accumulation.

Methods: We examined hepatic and adipose MBOAT7 expression in 119 obese patients and in experimental models. We silenced hepatic Mboat7 by i.v. administration of antisense oligonucleotides modified by morpholinos (MPO) for 4 consecutive days in C57B1/6 male mice ( $\mathrm{n}=6)$.

Results: In obese patients, hepatic mRNA levels of MBOAT7 progressively decreased from normal liver to simple steatosis and NASH $(\mathrm{p}<0.05)$. At multivariate analysis, type 2 diabetes $(\mathrm{p}<0.05)$, necroinflammation $(\mathrm{p}<0.01)$ and MBOAT7 genotype $(\mathrm{p}<0.01)$ were independently associated with MBOAT7 down-regulation. This suggests that down-regulation of hepatic MBOAT7 is involved in NAFLD pathogenesis independently of MBOAT7 genotype. In line with this hypothesis, we found that mRNA and protein levels of Mboat7 were reduced in experimental models of NAFLD: in the methionine-choline deficient diet (MCD), but more so in genetically obese Lep ${ }^{o b / o b}$ mice and in insulin resistant mice with Insulin receptor haplo-insufficiency (InsR+/-), characterized by hyperinsulinemia $(\mathrm{p}<0.05)$. Furthermore, in wild-type male mice Mboat7 was physiologically down-regulated by 
refeeding concomitantly with the rise of insulin levels and activation of hepatic insulin signaling through Phosphatidyl Inositol 3-Kinase (PI3K) and the Serine/Threonine Kinase AKT. In keeping with these results, activation of insulin-receptor dependent signaling down-regulated Mboat7 protein expression in primary mouse hepatocytes in a PI3Kinase-depedent manner. Consistently, in InsR+/hepatocytes insulin-mediated suppression of Mboat7 was lost $(\mathrm{p}<0.01)$. Moreover, the effect of insulin on Mboat7 expression was also abrogated in wild-type hepatocytes treated with antisense oligonucleotide stabilized by morpholination directed against FoxO1 (MPO) $(\mathrm{p}<0.05)$. These data suggest that FoxO1 is required to mediate the Mboat7 regulation by insulin. Consistently with this notion, the hampered Mboat7 expression observed in InsR+/- hepatocytes was rescued by FoxO1silencing by antisense-MPO ( $\mathrm{p}<0.05$ vs scramble). Finally, in vivo administration of antisense-MPO against Mboat7 for 4 consecutive days, induced a 45\% silencing of hepatic Mboat7 in wild-type male mice, which is comparable to that associated with the genetic risk variant, resulting in $80 \%$ increase in hepatic TG content $(\mathrm{p}<0.05$ vs scramble) and in microvesicular steatosis development. In contrast, it did not affect circulating glucose, insulin and lipid levels. Steatosis development was not explained by altered de novo lipogenesis or $\beta$-oxidation or VLDL export, but was associated with increased expression of fatty acids transporters (such as Fatp1, Fabp1). Conversely, Mboat7 silencing was not associated with alterations in the hepatic expression of inflammatory cytokines and chemokines (such as Tnf- $\alpha$ and Cxc110). These data are consistent with human genetic data suggesting that MBOAT7 down-regulation plays a causal role in NAFLD development.

Conclusion: In summary, we found that hyperinsulinemia, a typical feature of metabolic syndrome and post-prandial conditions, reduces hepatic MBOAT7 expression via increased insulin signaling activation through the Insulin receptor - PI3K - FoxO1 pathway. Reduced MBOAT7 then favors hepatic fat accumulation. These data suggest that MBOAT7 down-regulation may be implicated in the pathogenesis of progressive NAFLD. 


\section{Introduction}

\subsection{Nonalcoholic fatty liver disease}

Nonalcoholic fatty liver disease (NAFLD) is now the most frequent liver disorder worldwide, affecting $30 \%$ of adults in industrialized countries (1). NAFLD is characterized by hepatic fat accumulation that exceeds $5 \%$ of liver weight, in absence of alcohol abuse and it is strongly related to visceral obesity, type 2 diabetes mellitus (T2DM) and metabolic syndrome (MetS) (2, 3). Indeed, NAFLD is regarded as the hepatic manifestation of MetS and its prevalence increases with body mass index (BMI), reaching 60-70\% in obese patients (4).

NAFLD defines a wide spectrum of liver diseases, ranging from simple and uncomplicated steatosis to the progressive form, namely nonalcoholic steatohepatitis (NASH; 2-3\% of general population), which is characterize by hepatocyte ballooning, lobular necroinflammation, enhanced oxidative stress, mitochondrial dysfunction and liver fibrosis and that could evolve into cirrhosis and hepatocellular carcinoma (HCC) in susceptible individuals. Notably, hepatic fat accumulation and NASH have been recognized as risk factors for cirrhosis, liver failure and HCC, that is the third most common cause of cancer death (5). The risk of developing the progressive form of the disease is higher in patients with severe insulin resistance (IR) and T2DM, that are considered the strongest predictors of the progression of NAFLD to advanced fibrosis and cirrhosis. Indeed, the prevalence of NAFLD in T2DM patients is estimated at 34-74\% and it is even higher in T2DM patients with obesity (100\%) (6).

To date, there are no approved drugs for the treatment of NAFLD and lifestyle intervention with a focus on healthy eating, weight loss and regular exercise remain the mainstay in the management of patient with NAFLD. Indeed, effective and sustained weight loss has been associated with marked improvement in glycemic control, hepatic insulin sensitivity, liver enzymes and liver histology (7). 


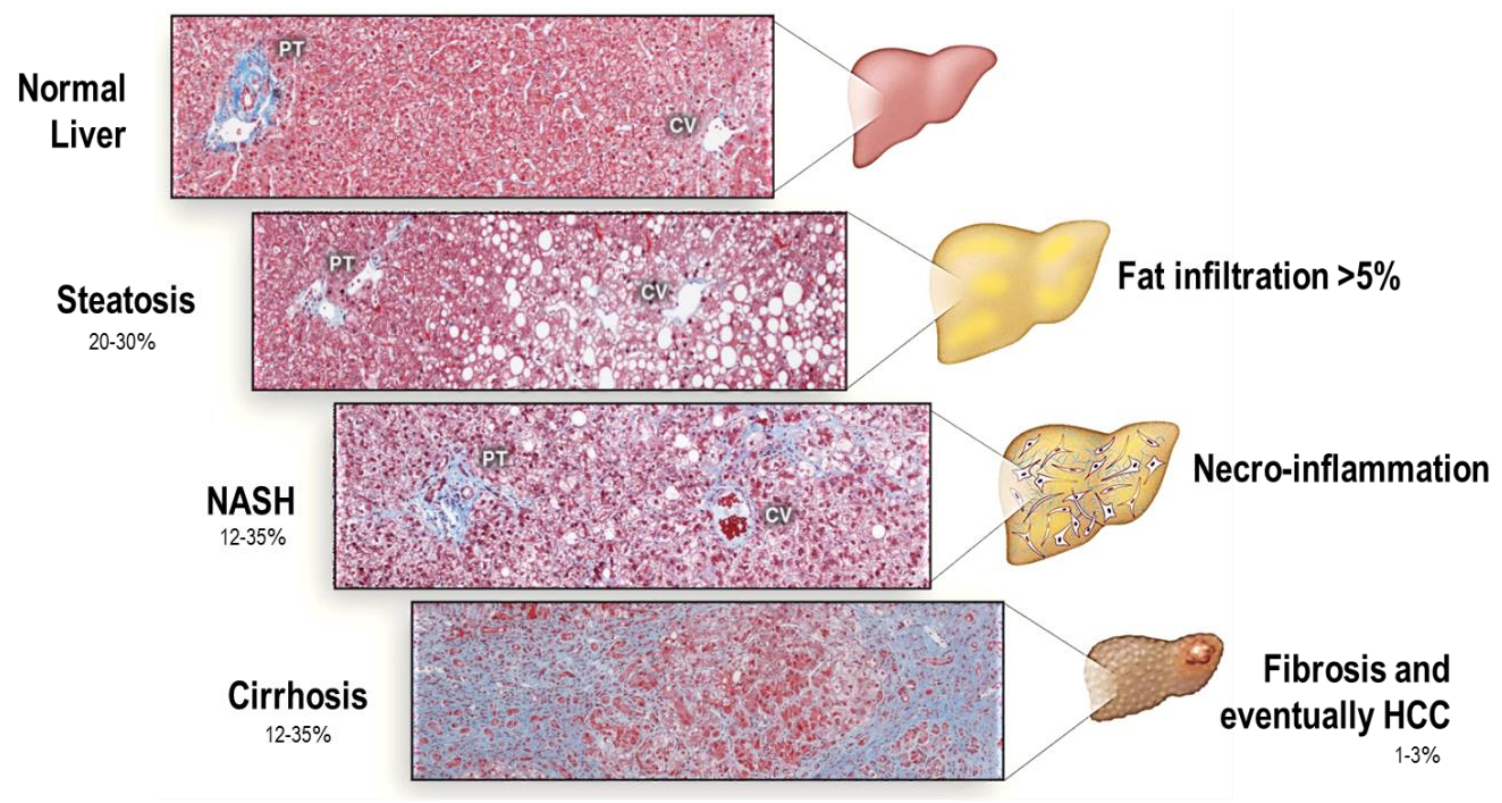

Fig. 1: The spectrum of NAFLD. The accumulation of TG within lipid droplets in hepatocytes causes steatosis. Steatosis associated with inflammation, cell death, and fibrosis is referred to as NASH, which can progress to cirrhosis. Individuals with cirrhosis have an increased risk of HCC. Modified from Cohen C. et al, Science 2011 (8).

\subsection{Insulin resistance and NAFLD pathogenesis}

Although the exact mechanism of NAFLD pathogenesis is still not completely understood, current knowledge supports a model whereby development of liver damage is multifactorial, commonly summarized to as the 'multi-hit' hypothesis (9-12). The 'first hit' causes the rising of hepatic steatosis due to IR.

Mammals have complex mechanisms to maintain energy homeostasis during periods of nutritional abundance and starvation. The nutritional state of an organism is reflected by the circulating levels of the two pancreatic hormones insulin and glucagon, which elicit regulatory responses in the peripheral tissues (adipose tissue and muscle). Liver acts as a 'metabolic integrator', by regulating 
glycogen degradation, gluconeogenesis, fatty acids oxidation and ketogenesis, thereby maintaining normo-glycemia and ensuring constant energy supply to glucose-dependent tissues, such as the brain. Following food intake, plasma insulin levels rise and suppress hepatic glucose production, $\beta$-oxidation and ketogenesis and instruct the liver to convert glucose to glycogen for storage and promote TG synthesis (13). In hepatocytes, metabolic responses to changes in plasma insulin levels are mediated at intracellular levels by the insulin/phosphatidylinositol-3-kinases (PI3K)/Akt signaling cascade. Downstream targets of the Akt kinases are glycogen synthase kinase 3 (GSK3), mammalian target of rapamycin (mTOR) and several members of the Forkhead box transcription factors (14), such as Forkhead box protein O1 (FoxO1), an activator of rate-limiting gluconeogenic enzymes (15) and FoxA2, a key regulator of hepatic lipid metabolism, that is transcriptionally active in the fasted state $(16-18)$.

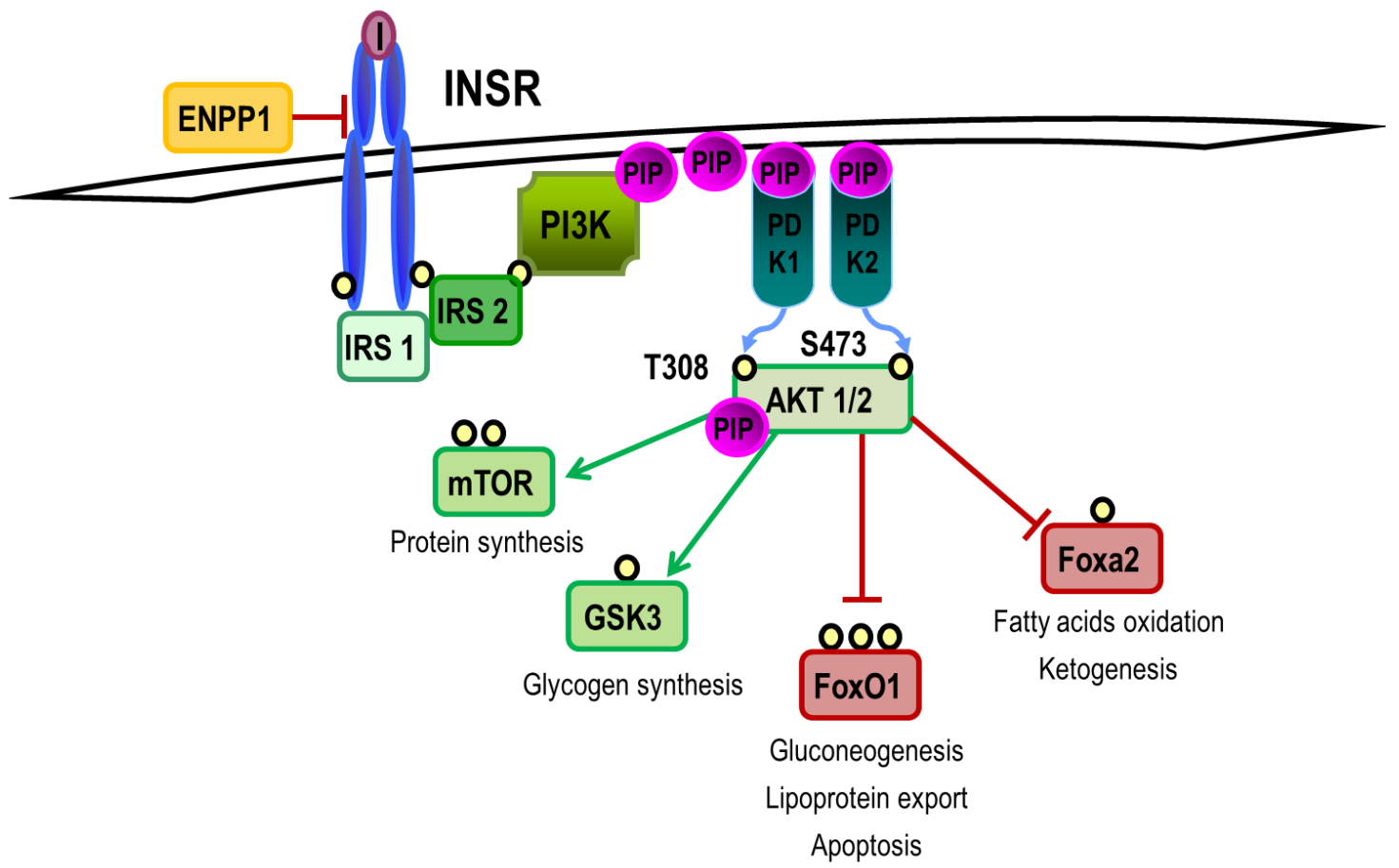

Fig. 2: The insulin signaling pathway in hepatocytes. Insulin binding to its receptor (INSR) leads to autophosphorylation of the insulin-receptor substrates (IRS1 and 2), activation of PI3K and subsequent phosphorylation of Akt 1/2. Activation of protein kinase Akt 1/2 mediates the metabolic effects of insulin 
promoting glycogen and protein synthesis, through GSK3 and mTOR and inactivating the transcription factors FoxO1, which in absence of insulin induces gluconeogenesis, lipoprotein export and apoptosis and FoxA2, involved in fatty acids oxidation and ketogenesis. Phosphorylation of these two transcription factors in response to Akt 1/2 activation leads to their nuclear exclusion, ubiquitination and proteasomal degradation (19). Modified from Dongiovanni P. et al, Expert Review of Gastroenterology and Hepatology 2016 (20).

In peripheral metabolic tissues, muscle and fat, insulin stimulates dietary glucose uptake by driving the translocation of glucose transporters GLUT-4 to the cell surface and promotes fat storage through induction of lipoprotein lipase and lipogenesis.

IR is defined as a clinical condition where normal or elevated insulin levels produce an attenuated biological response, particularly in terms of glucose homeostasis (21). IR may be caused directly from altered insulin receptor activity or by alteration in the downstream signaling pathways. It is rarely determined by loss-of-function mutation in INSR gene, but most frequently reflects reduced expression on the cellular membrane. In obesity, IR is described by hyperinsulinemia by decreasing insulin clearance, increased glucose output and decreased glucose utilization of the liver. Indeed, in order to compensate the impaired insulin response, the pancreatic $\beta$-cells increase insulin production, eventually undergoing exhaustion and leading to impaired glucose tolerance and T2DM (22). In particular, in IR conditions, insulin fails to inactivate FoxO1 further inducing hepatic glucose output (23). Conversely, the hepatic regulation of FoxA2 phosphorylation by insulin-stimulated IRS signaling is not impaired in hyperinsulinemia or IR states, where FoxA2 is found exclusively in the cytoplasm of hepatocytes, despite the defect in hepatic insulin signaling $(17,24)$. This difference can be explained by the preferential phosphorylation of FoxO1 through insulin-IRS2 signaling, which is impaired in 
hepatic IR, whereas FoxA2 can be phosphorylated by either insulin-IRS1 or insulin-IRS2 signaling (17).

In muscle and adipose tissue, IR determines an impairment of glucose uptake and promotes an enhanced activity of hormone-sensitive lipoprotein lipases, favoring lipid storage dismissal.

Thus, in NAFLD patients, hepatic fat accumulation results from an increased efflux of nonesterified or free fatty acids (FFAs) from adipose tissue to the liver (25). FFAs which derive from peripheral lipolysis related to adipose tissue IR (26) are stored into the hepatocytes as triglycerides (TG), in order to protect hepatocytes themselves from lipotoxicity (25). Furthermore, hyperinsulinemia activate also lipogenic transcription factors, such as sterol regulatory element-binding protein-1c (SREBP-1c) and induce de novo lipogenesis from glucose, exacerbating hepatic fat deposition. Reduction in neutral lipid secretion through very low-density lipoproteins (VLDL) (27) and in $\beta$ oxidation due to mitochondrial damage are also involved in steatosis exacerbation. Thus, the hepatic fat accumulation derives from an unbalance between TG acquisition, synthesis and removal (8). Viceversa, fatty liver per se may then precipitate hepatic IR promoting metabolic disturbances and cardiovascular damage $(28,29)$. Indeed, excess of intracellular storage of lipid metabolites, such as diacylglycerol, ceramides and long chain acyl CoA, has also been implicated as a mediator of IR, triggering the activation of c-Jun $\mathrm{N}$ terminal kinase (JNK), affecting insulin signaling (30).

\subsection{Evolution to NASH}

The progression of steatosis to NASH is currently explained by the occurrence of multiple parallel hits in the background of fatty liver. These insults are represented by a) direct hepatic lipotoxicity due to toxic lipid metabolites; b) hepatocellular oxidative stress secondary to free radicals

produced during $\beta$ - and $\omega$ - oxidation of FFAs and subsequent activation of stress related signaling 
pathways; c) inflammation triggered by endotoxin engaging TLR-4 receptors in Kupffer cells, (macrophages localized into the sinusoids) and in hepatocytes due to increased intestinal permeability and altered intestinal flora $(31,32)$; d) qualitative and quantitative changes in the gut microbiota; e) pro-inflammatory cytokines and chemokines release (33) and f) injury occurring to intracellular organelles (endoplasmic reticulum (ER) stress and mitochondrial disfunction). These injuries also trigger to fibrogenesis and progression to cirrhosis. In fact, cytokines and chemokines are involved in the recruitment and activation of Kupffer cells and in the transformation of hepatic stellate cells (HSCs) into myofibroblasts, both involved in the progression from steatosis to NASH (34).

Visceral obesity also contributes to NASH development as well as IR. Several adipokines are released by white adipose tissue (WAT), such as adiponectin, leptin, resistin, TNF- $\alpha$, IL-1 $\beta$, IL-6 (9). These adipokines down-regulate glucose transporter GLUT-4 expression via increased serine phosphorylation of IRS-1 (14) and affect insulin signaling, inducing IR (35).

The worsening of liver damage is marked by accumulation of hepatic fibrosis resulting from deposition of extra-cellular matrix (ECM) preferentially in perisinusoidal/pericellular spaces, due to the activation of HSCs. HSCs are localized in sinusoids adjacent to the hepatocytes; they are generally quiescent and characterized by the presence of retinol droplets in the cytoplasm. When activated by hepatotoxic insults, HSCs acquire the ability to proliferate, contract themselves and migrate, produce cytokines and chemokines, release retinols and secrete collagen and ECM, perpetuating the inflammation and fibrosis. In NASH patients, collagen fibers are mainly localized around the hepatocytes, hesitating in perisinusoidal/pericellular ("chicken wire") fibrosis, which spreads into the parenchyma. Hyperinsulinemia and hyperglycemia stimulate HSCs to produce ECM. IR correlates with the severity of liver fibrosis, reducing ECM degradation and favoring collagen cross-linking, and fibrosis progression is observed in T2D patients with NAFLD (36). Moreover, functional common genetic variants of molecules involved in insulin signaling have been associated with the severity of 
liver damage (37). There is a strong correlation between IR severity and the development of NASH, but it is still unknown to what extent IR is cause or consequence of liver damage, and the precise molecular mechanism underlying the association (38).

Activated HSCs could have two different fates: apoptosis or senescence. About half of activated HSCs escape apoptosis. They down-regulate fibrogenic genes, but they could be easily reactivated, in response to recurring fibrogenic stimuli (39). Cellular senescence of persistent HSCs in response to chronic inflammation contributes to the pathogenesis of HCC in NASH subjects. Indeed, senescence is linked to gene expression alterations, including a program known as the senescence-associated secretory phenotype (SASP) (40). In this program, a large amount of cytokines, chemokines and proteases secreted from senescent cells promote crosstalk with other cells in the microenvironment. Some of SASP factors have a positive function in resolution of liver damage, promoting cell-cycle arrest or selective apoptosis of activated HSCs, through immune cells recruitment. Others factors, instead, are associated with inflammation and tumorigenesis promotion (as IL-1 $\beta$, IL-6, CXCL1, CXCL9 and PAI-1) (41).

\subsection{Genetic predisposition in NAFLD and NASH}

Although obesity and IR are the most prevalent risk factors for NAFLD, hepatic fat content varies substantially among individuals with equivalent adiposity, indicating that other factors contribute to this condition. Indeed, epidemiological, familial, and twin studies provide evidence of a strong heritability of hepatic fat content and there is a huge inter-ethnic variability in the predisposition towards NAFLD $(42,43)$. Two large multi-ethnic population studies conducted in the US detect higher risk of NAFLD in Hispanics than individuals of European descent, whereas African-Americans are 
protected irrespectively of diabetes, excess in body weight and socioeconomic factors, consistent with a key role of heritability (44).

Several genetic risk factors are recognized to influence the onset and the severity of NAFLD. To date, the major genetic determinant of inter-individual and ethnicity-related differences in hepatic fat content and the major predictor of predisposition to progressive NAFLD, is the rs738409 C>G variant of the Patatin-like Phospholipase domain containing 3 gene (PNPLA3 or adiponutrin), encoding for the amino acid substitution Isoleucine to Methionine at the position 148 (referred to as I148M) (45). The M risk allele is more prevalent in Hispanics than in Europeans and less frequent in African Americans, possibly explaining the inter-ethnic susceptibility to NAFLD. PNPLA3 is a intracellular membrane lipase, localized in the endoplasmic reticulum and at the surface of lipid droplets, in hepatocytes, adipocytes, and in HSCs. In particular, it is involved in lipid remodeling on lipid droplet surface, retinol metabolism and retinol release by HSCs $(46,47)$. The I148M variant has a strong impact on the full spectrum of liver damage related to fatty liver, encompassing NASH, advanced fibrosis, and $\mathrm{HCC}$, and influences the response to therapeutic approaches (48). The mechanism of steatosis development is related to accumulation of the mutated protein on the lipid droplet surface, thus, interfering with lipid remodeling in fatty-laden hepatocytes and inhibiting the activity of other lipases and accordingly, reducing TG turnover and dismissal. The accumulation of the I148M mutated protein seems to be due to the less accessibility to ubiquitin ligases and to impaired proteasomal degradation $(49,50)$. In HSCs, instead, PNPLA3 I148M variant alters retinol secretion, potentially contributing directly to fibrogenesis and carcinogenesis, increasing the risk in cirrhosis and HCC development, independently of the predisposition to steatosis (51-53). The size effect of the I148M variant on the risk of NAFLD is the strongest ever reported for a common variant, modifying the genetic susceptibility of NAFLD and liver disease progression. 
The low-frequency rs58542926 C>T variant of Transmembrane 6 superfamily member 2 gene (TM6SF2), encoding for the loss-of-function E167K substitution also predisposes to progressive NAFLD by altering the secretion of VLDL. This is associated with hepatic fat content, aminotransferases concentration and lower serum lipoproteins. The mechanism is related to retention of lipids within intracellular lipid droplets resulting in steatosis. Conversely, by reducing circulating lipids, the E167K variant decreases the risk of cardiovascular events $(54,55)$.

The common loss-of-function polymorphism (rs1260326) in the Glucokinase regulatory (GCKR) gene, encoding for the P446L substitution has been also associated with increased fasting TG levels, hepatic fat accumulation and liver damage. GCKR regulates DNL by controlling the influx of glucose into the hepatocytes and the P446L variant affects GCKR ability to negatively regulate glucokinase, determining a constitutive activation of hepatic glucose uptake. This leads to decreased circulating fasting glucose and insulin levels, favoring, in turn, glycolysis and steatosis onset by providing malonyl-CoA as substrate for DNL and by blocking fatty acid oxidation (56-58).

\subsection{The MBOAT7 rs641738 variant is novel a risk factor for NAFLD onset and progression}

Recently, the first genome-wide association study (GWAS) screening the determinants of alcoholic cirrhosis in heavy drinkers, led to the identification of the common variant rs641738 C>T (population prevalence of 58-67\%), a single nucleotide polymorphism in the Membrane bound oacyltransferase domain-containing 7 - Transmembrane channel-like 4 (MBOAT7-TMC4) as a susceptibility locus on chromosome 19 (59). Most importantly, we have shown that this variant associates with steatosis severity and the entire spectrum of liver damage related to NAFLD, particularly in terms of necroinflammation and more advanced fibrosis $(60,61)$. The impact of this 
variant on hepatic fat accumulation and metabolism has been demonstrated in two different independent cohorts of individuals: the Dallas heart study (DHS) cohort and the Liver biopsy crosssectional cohort. The DHS cohort is a population study that includes 3854 individuals, of whom 2736 underwent to proton magnetic resonance spectroscopy to measure hepatic TG content. The Liver biopsy cross-sectional cohort (LBC), instead, comprehends 1149 European subjects, who underwent to liver biopsy for suspected NASH or severe obesity. Patients carrying $\mathrm{T}$ allele show an enhanced intrahepatic TG content in these two independent cohorts and a higher prevalence of NASH and fibrosis in the LBC cohort, compared with non-carriers (60). Furthermore, children carrying the T allele of MBOAT7 have higher plasma ALT levels, C-reactive protein (CRP) concentration and body fat percentage than the non-carriers (62).

In addition, the MBOAT7 variant has been recognize also as a risk factor for HCC predisposition, in patients without cirrhosis. In fact, in 765 Italian NAFLD patients without advanced fibrosis, it has been associated with NAFLD evolution to HCC (63). Nonetheless, the rs641738 variant has been identified as a risk factor for liver damage progression, in particular in the transition to early fibrosis, even in viral hepatitis $\mathrm{C}(\mathrm{HCV})$ and $\mathrm{B}(\mathrm{HBV})(64,65)$. Finally, in 1121 non-cirrhotic patients with HCV or alcoholic liver disease, the T allele has been independently associated with HCC risk. The effect of this variant on HCC risk disappears with disease progression and it has no effect on the late stages of fibrosis (63). 


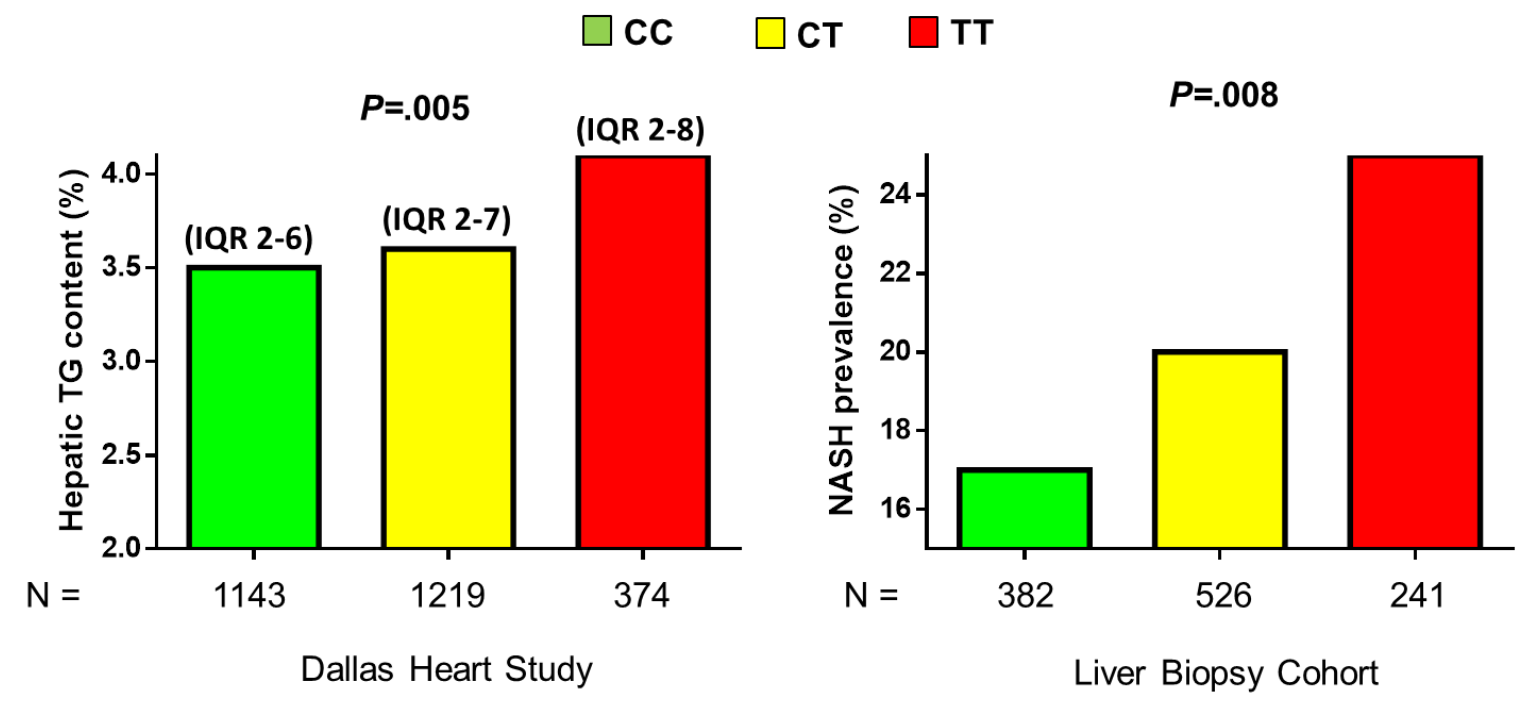

Fig. 3: The rs641738 variant is associated with increased intrahepatic TG content in the DHS cohort and with the severity of histologic damage in the Liver Biopsy Cross-Sectional Cohort. The association between TG content and $\mathrm{T}$ allele presence has been tested by linear regression analysis adjusted for age, sex, ethnicity, BMI, IFG/T2DM, PNPLA3 I148M, and TM6SF2 E167K genotype, and data are shown as median value. Numbers in brackets represent interquartile range. $\mathrm{P}$ value represents the significance of a linear trend in median trait value among genotypes. The association between the rs641738 variant and NASH prevalence has been tested by multivariate ordinal regression analysis adjusted for age, sex, BMI, presence of IFG/T2DM, number of PNPLA3 I148M alleles, presence of the TM6SF2 E167K variant, and indication of liver biopsy (severe obesity vs nonalcoholic fatty liver with increased liver enzymes). $\mathrm{P}$ values represent the significance of a trend in the prevalence of NASH among genotypes. Genetic analyses have been performed by using an additive model. CC, homozygotes for the $\mathrm{C}$ allele; CT, heterozygotes; TT, homozygotes for the $\mathrm{T}$ allele.

\subsection{MBOAT7 function and pathogenic effect of the rs641738 variant}

MBOAT7 is the mammalian orthologue of mboa-7, that has been previously identified by using a RNA interference-based genetic screen in Caenorhabditis elegans (66). mboa-7 deletion mutants showed a decrease in the content of Eicosapentaenoic acid (EPA), the predominant polyunsaturated 
fatty acid (PUFA) in C. elegans, in phosphatidylinositol (PI) amount (66) and impaired PI-3-phosphate (PI3P)-related events such as early endosome morphology and autophagy (67).

MBOAT7 is also known as lysophosphatidylinositol acyltransferase 1 (LPIAT1). It codifies for an enzyme, member of the "Lands' Cycle" of phospholipid acyl-chain remodeling of the membranes, through sequential deacylation and reacylation reactions. It catalyzes a desaturation of the second acylchain of phospholipids and specifically transfers a PUFA, in form of acyl-CoA to lysophosphatidylinositols (lysoPI) and other lysophospholipids, using as preferential substrate the arachidonoyl-CoA. Thus, it regulates the amount of free arachidonic acid, a potent trigger for liver inflammation and fibrosis, that is also the precursor of the main proinflammatory mediators (Eicosanoids) (68). Indeed, MBOAT7 activation has been associated with anti-inflammatory processes by limiting the availability of free arachidonic acid in Leukotriene B4 synthesis in neutrophils, that is a strong chemoattractant mediator (69). Furthermore, it may also influence signal transduction pathways and the fluidity and the dynamism of cell membranes, essential for membrane fusion and fission steps during endocytosis, exocytosis, cytokinesis and vesicle trafficking.

Mboat7 plays a crucial role in brain development in mice, since arachidonic acid is the most enriched PUFA in the brain and it is involved in multiple aspects of neuronal development and function. Mboat7 knock-out (KO) mice show almost no activity with arachidonoyl-CoA as an acyl donor and show reduced arachidonic acid contents in PI and PI phosphates (PIP, PIP2 and PI3P). PI are lipids, which regulate membrane dynamics and signal transduction pathways, whereas PI phosphates are synthesized by PI kinases and phosphatases and play crucial roles in the regulation of a wide variety of cellular processes via specific interactions of PIP-binding proteins (70, 71). Among PI phosphates, PI 3-phosphate (PI3P) regulates a variety of vesicular trafficking pathways, including endocytosis, endosome-to-Golgi retrograde transport, autophagy and mTOR signaling pathway (72). PI consist of a glycerol backbone and two variable fatty acyl-chains, one of which is predominantly 
saturated and the other polyunsaturated. Specifically, arachidonic acid-containing PI/PI phosphates play an important role in normal cortical lamination during brain development in mice. Indeed, Mboat7 KO mice die within a month and show atrophy of the cerebral cortex and hippocampus, disordered cortical lamination and neuronal processes and delayed neuronal migration in the cortex (67, 73). Notably, inactivating variants in MBOAT7 lead to intellectual disability accompanied by epilepsy and autistic features in patients $(74,75)$.

At a cellular level, MBOAT7 is highly expressed in human hepatocytes, sinusoidal endothelial cells, immune cell subsets and HSCs. It is localized specifically in membrane fractions rich in phospholipids, such as the ER and the mitochondria-associated membranes (MAM), where arachidonic acid-selective acyl-CoA synthases are enriched (60). MAM is a membrane bridge between the ER and mitochondria, that is involved in the biosynthesis and trafficking of lipids between the two organelles (76, 77) and in lipid droplets formation (78). It interacts with the small subunit of serine palmitoyltransferase a (ssSPTa), that plays a role in fatty acid remodeling of PI, probably by facilitating the MAM localization of MBOAT7 (79).

Another enzyme of the MBOAT family, MBOAT5, also named Lysophosphatidylcholine acyltransferase 3 (LPCAT3), has similar enzymatic activity to MBOAT7. It is ubiquitously expressed, especially in liver, testis, kidney, pancreas and adipose tissue, where it participates in the acyl-chain remodeling of Phosphatidylcholines (PC), Phosphatidylserine (PS) and Phosphatidylethanolamines (PE) (80). Expression of LPCAT3 mRNA is controlled by liver X receptors (LXR) and is induced during adipogenesis (81). Mboat5 KO mice are neonatally lethal due to an extensive TG accumulation in enterocytes, because TG are not assembled into lipoproteins. Mice with specific deletion of hepatic Mboat5 display a decreased arachidonic acid-containing PC, PS and PE in the liver and an increased risk of hepatic steatosis onset, due to altered lipid kinetics within hepatocytes, and inflammation (82, 
83). Conversely, the induction of Mboat5 ameliorates saturated free fatty acid-induced ER stress, lowering also blood glucose and insulin levels in Lep ${ }^{o b l o b}$ mice $(81,84)$.

MBOAT5 and MBOAT7 are both susceptible to inhibition by Thimerosal, a thiol-reactive reagent.

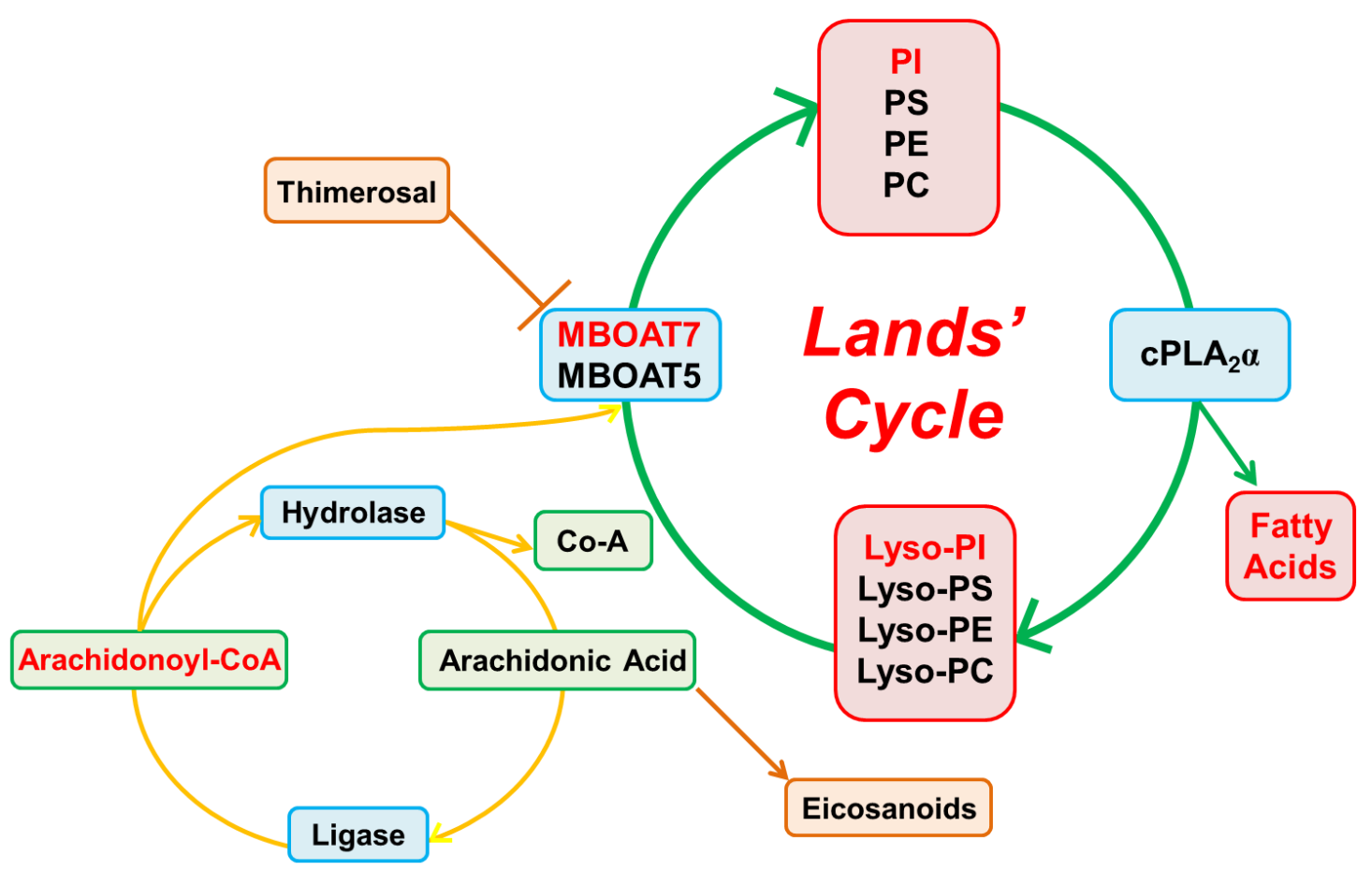

Fig. 4: Integrated model for the Land's cycle of phospholipids remodeling of the membranes and arachidonic acid mobilization.

The major phospholipids in the membranes are Phosphatidylcholines (PC), Phosphatidylserine (PS), Phosphatidylethanolamines (PE) and Phosphatidylinositols (PI). Arachidonic acid in the sn-2 position of these membrane lipids is released by the action of Phospholipase A2 (cPLA2), generating the corresponding lysophospholipids and free arachidonic acid. This can then be used for the production of various proinflammatory Eicosanoids or can be returned to the phospholipid pool via the action of an acyl-CoA ligase and either MBOAT7 (that uses lyso-PI) or MBOAT5 (that uses lyso-PC, lyso-PS, and lyso-PE). Thimerosal, an MBOAT inhibitor, increases the production of Eicosanoids (Prostaglandins and Leukotrienes). 
The pathogenic effect of the rs641738 variant is probably due to the dampened gene and protein expression of MBOAT7 in the liver of patients carrying $\mathrm{T}$ allele. In line with these results, these patients also display changes in plasma and hepatic PI species, specifically a decrease in PI enriched in omega-3 PUFA and arachidonic acid, consistently with decreased hepatic MBOAT7 total activity (60, 61). The risk $\mathrm{T}$ allele is associated with a PI composition pattern suggestive of lower levels of arachidonoyl-PI/total PI (PI containing polyunsaturated fatty acid chains) and with higher levels of oleyl-PI/total PI linoleoyl-Pl/total PI ratios (PI containing saturated and monounsaturated fatty acid chains). These data are consistent also with the changes found in liver PI levels of MBOAT7 KO mice, suggesting that reduced enzymatic activity of MBOAT7 may explain the increased liver fat accumulation and progressive liver damage, in $\mathrm{T}$ allele carriers. However, to date it is not known whether rs641738 causes the decreased expression of MBOAT7 or whether the observed reduction in both mRNA and protein synthesis is due to linkage disequilibrium between rs641738 and variants in the 3'UTR of MBOAT7 (eg, rs8736 C>T, $\mathrm{R}^{2}>0.95$ in the CEU population from the 1000 genomes project), which may influence mRNA stability/translation. Specifically, in severely obese patients, the rs641738 variant is in high linkage with the MBOAT7 $3^{\prime} \mathrm{UTR}$ variant rs8736 polymorphism $\left(\mathrm{R}^{2}=0.98\right.$; only $1 / 98$ discordant case). The rs 8736 polymorphism is non-significantly more closely associated with NAFLD and MBOAT7 expression than rs641738, suggesting that rs641738 is not the causal variant, but may be in linkage with variants influencing MBOAT7 expression (63). 


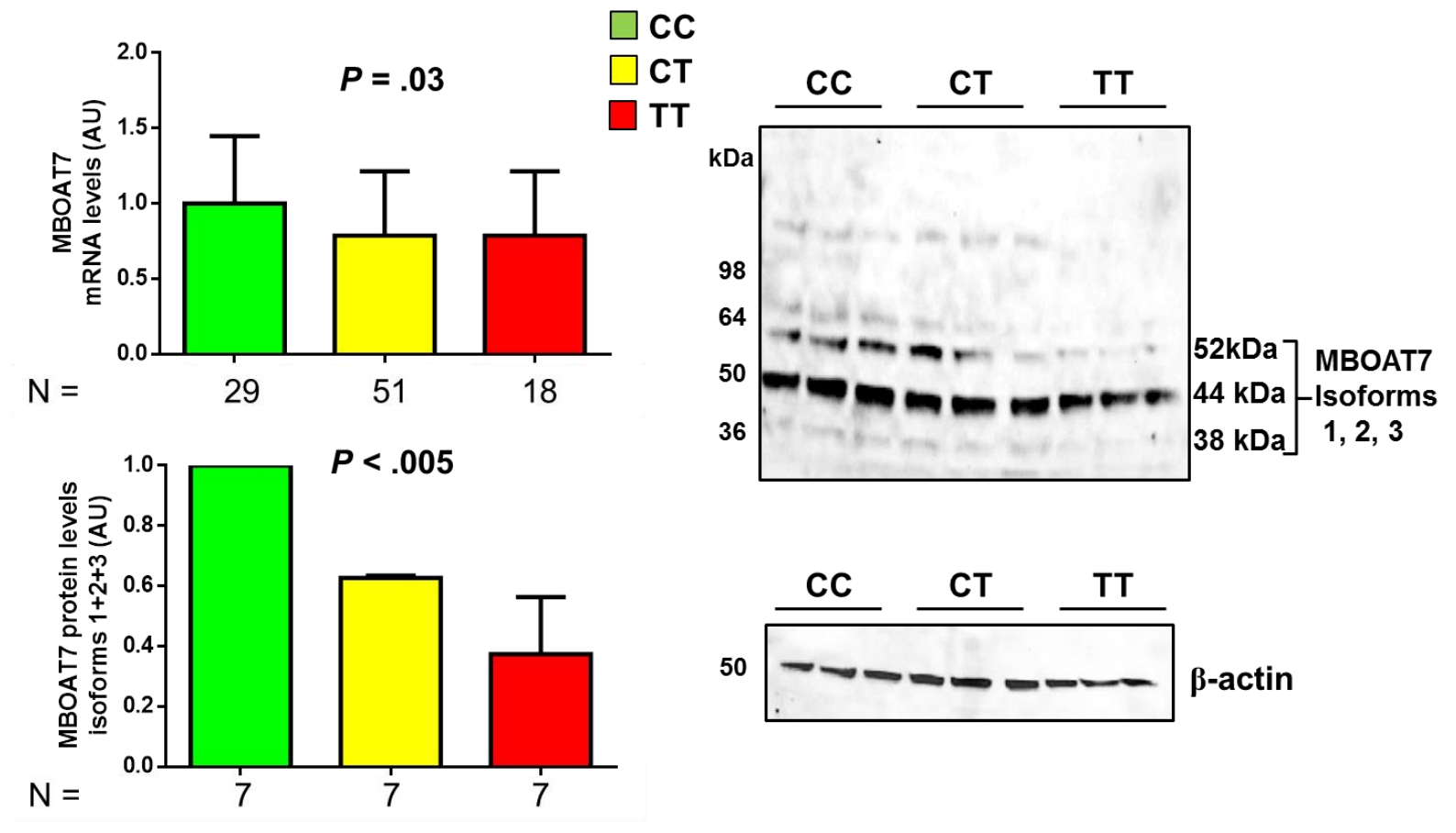

Fig. 5: The rs641738 variant decreases MBOAT7 mRNA expression and synthesis level in liver biopsies of obese patients. The rs641738 hepatic mRNA levels of MBOAT7 in 98 severely obese patients are stratified by the presence of the rs643718 $\mathrm{C}>\mathrm{T}$ genotype and data are expressed as fold increase as compared with the protective CC genotype. Western blotting analysis and quantification, instead, represents MBOAT7 protein levels of 7 samples of obese patients for each rs641738 genotype. Data are expressed as fold increase of the 3 MBOAT7 isoforms 1-3 (molecular weight: $52 \mathrm{kDa}, 44 \mathrm{kDa}$, and $38 \mathrm{kDa}$ ), as compared with the protective CC genotype. P $<.005$ (additive model; it represents the difference in the traits examined across MBOAT7 genotypes). 


\section{Aims}

Nonalcoholic fatty liver disease (NAFLD) is now the most frequent liver disorder worldwide, affecting around $30 \%$ of adults in industrialized countries (1). The rs641738 $\mathrm{C}>\mathrm{T}$ variant in the membrane-bound O-acyltransferase domain-containing protein 7 (MBOAT7) gene has been recently found to be associated with increased risk of developing steatosis and progressive form of NAFLD in adults. MBOAT7, also known as lysophosphatidylinositol acyltransferase 1 (LPIAT1), is an enzyme that is expressed in the liver and is involved in the reacylation of phospholipids as part of the phospholipid remodeling pathway 'Land's cycle' by specifically transferring polyunsaturated acylCoAs, such as arachidonoyl-CoA, to lysophosphatidylinositol and other lysophospholipids. The carriers of the T allele of the MBOAT7 polymorphism were found to have reduced expression and synthesis of the MBOAT7 protein in the liver and changes in plasma phosphatidylinositol species consistent with decreased MBOAT7 function $(60,61)$. These observations suggest that reduced enzymatic activity of MBOAT7 may explain, on one side, the increased liver fat accumulation and the steatosis onset, and, on the other side, the liver damage worsening into NASH and advance fibrosis, in $\mathrm{T}$ allele carriers.

Our hypothesis is that the blunted expression of MBOAT7 due to the genetic background and insulin resistance plays a causal role in the development and progression of NAFLD. Indeed, higher levels of circulating bioactive proinflammatory metabolites of arachidonic acid have been reported in patients with NASH.

Thus, the aims of this study were

1) To examine the clinical and genetic determinants of hepatic MBOAT7 expression in patients at risk of NASH and the association with liver damage. To this end, we evaluated 
the hepatic and adipose tissue MBOAT7 expression in 119 Italian severely obese patients at risk of NASH, who underwent liver biopsy during bariatric surgery.

2) As we detected a strong link between Mboat7 down-regulation and hyperinsulinemia associated with insulin resistance, to investigate in vivo and ex vivo in primary mouse hepatocytes and cell lines the mechanisms by which insulin signaling regulated MBOAT7 expression during physiological conditions and in dietary and genetics models of NAFLD.

3) To test the causative role of Mboat7 down-regulation in NAFLD development in experimental models, confirming data from genetic epidemiological studies. To this end, we silenced hepatic Mboat7 in wild-type mice in vivo, by i.v. administration of antisense oligonucleotides modified by morpholinos (MPO), and evaluated the impact on hepatic fat content and metabolism and inflammation. 


\section{Material and methods}

\subsection{Chemicals}

Methionine and Choline deficient diet (MCD) was purchased from Test Diet (London, UK); insulin Insuman Rapid (0.75 U/Kg) was ordered from Sanofi Aventis (Milan, Italy). Anti $\beta$-Actin was acquired from St. Cruz Biotechnologies (Santa Cruz, United States); anti P-Thr308 Akt, Akt, FoxO1, P(T389)-P70S6, Rapamycin and Akt inhibitor (LY294002) were purchased from Cell Signaling Technologies (Boston, United States); anti Foxa2 and anti Histone H3 antibodies were purchased from Abcam (Cambridge, UK); anti-MBOAT7 from Sigma-Aldrich. Dulbecco's modified Eagle's medium (DMEM), fetal bovine serum (FBS), phosphate-buffered saline (PBS), L-Glutamine, Penicillin/Streptomycin, Trypsin/EDTA and Hank's balanced salt solution (HBSS) were obtained from Gibco-ThermoFisher scientific (Waltham, United States). Fast SYBR green master mix was obtained from Life Technologies-ThermoFisher Scientific (Waltham, United States). Collagenase type IV from Clostridium Histolyticum, Pronase E from Streptomyces Griseus, DNAse I from bovine pancreas, Optiprep density gradient media and fructose were purchased from Sigma-Aldrich (St. Louis, United States). Clarity western ECL substrate was obtained from Bio-Rad Laboratories (Hercules, United States). Free fatty acids (FFAs) Quantification Kit, Triglyceride Quantification Kit was purchased from BioVision (Milpitas, United States). Rat/Mouse Insulin ELISA kit (Millipore, United States) was bought from Merck Millipore (Billerica, United States).

\subsection{Experimental models}

C57Bl/6 mice (Charles River, Calco, Italy) were housed at constant room temperature $\left(23^{\circ} \mathrm{C}\right)$ under 12-hour light/dark cycles with ad libitum access to water in compliance with the Principles of 
Laboratory Animal Care (NIH publication 86-23). InsR+/- mice on C57B1/6 background were kindly provided by Prof. Accili D, Columbia University, NY, USA (85). Genotyping was performed using genomic DNA extracted from mouse tail (86). C57B1/6 Lep ${ }^{o b / o b}$ mice were purchased from Charles Rivers (Calco, Italy).

To induce NASH, wild-type and InsR+/- male mice, which are more susceptible to develop liver damage and fibrosis compared to female, were fed either MCD (Methionine Choline deficient diet, $14.8 \%$ protein, $12.4 \%$ fat and $72.8 \%$ carbohydrates, Test Diet, London, UK) or regular chow (15.1\% protein, $12.4 \%$ fat and $72.4 \%$ carbohydrates Test Diet, London, UK) for 6 weeks, starting from 6 weeks of age. Experiments were conducted in 10 mice per group. In a second set of experiments, supplemental fructose was administered as $30 \%$ fructose in drinking water. Body weight was recorded weekly throughout the experiments. Before sacrifice, mice were fasted for 16 hours and the interventions were done during the light cycle.

In another experimental setting, in order to study the physiological alteration of Mboat7 due to the nutritional state, wild-type male mice of 6 weeks of age were fasted for 16 hours and then refed for different time point (15 minutes, 1 hours, 4 hours or 8 hours). Experiments were conducted in 7 mice per group per each time point, unless otherwise specified.

The experimental protocol was approved by University of Milan and Italian Ministry of Health Review Boards (protocol 8/14).

\subsection{Samples collection and biochemical evaluation}

Blood samples were collected by tail vein puncture. Blood glucose was measured by an AccuCheck glucometer (Roche Diagnostics AVIVA, Mannheim, Germany) and fasting plasma insulin levels by ELISA (EZRMI-13K, Millipore, Billerica, MA). Plasma and hepatic triglycerides, total 
cholesterol and alanine aminotransferase (ALT) levels were measured with an automated analyzer (CobasC 702, Roche, Switzerland). Circulating free fatty acids (FFAs) were measured by a colorimetric assay (BioVision, Milpitas, United States) according to the manufacturer's instructions. At sacrifice, liver, proximal intestine and visceral adipose tissue (VAT) samples were rapidly removed, harvested and snap frozen in liquid nitrogen. Samples of liver were fixed in $10 \%$ PBS buffered formalin and embedded in paraffin within 24 hours of formalin fixation, for histological evaluation. Tissue sections were stained by Hematoxylin and Eosin (H\&E) to assess liver pathology, according to Kleiner et al (87).

\subsection{Gene silencing}

To silence ex vivo FoxO1 and/or Foxa2, key mediators of insulin signaling at transcriptional level, we exploited Morpholino antisense oligonucleotides (MPO) specifically targeting FoxO1 and/or Foxa2 by blocking translational initiation and the in vivo "Standard control", whose sequences were 5'CCACCAGCAGAGAAGTACCGGGAGA-3', 5'-TCCATCTTCACGGCTCCCAGCATAC-3' and 5'CCTCTTACCTCAGTTACAATTTATA-3' respectively (Gene tools, www.gene-tools.com). Primary mouse hepatocytes were incubated with FoxO1 and/or Foxa2-specific MPO or scramble $(10 \mu \mathrm{M})$ for 24 hours in medium $0.5 \%$ BSA. Moreover, InsR+/- mice were treated with $12.5 \mathrm{mg} / \mathrm{kg}$ of FoxO1specific MPO or scramble administered i.v. once a week during 3 weeks of MCD feeding ( $\mathrm{n}=5$ per group).

To silence in vivo Mboat7 wild-type mice were treated with $12.5 \mathrm{mg} / \mathrm{kg}$ of MBOAT7-specific MPO or scramble administered i.v. daily for 4 days ( $n=6$ per group). MBOAT7-specific MPO sequence was 5'-ATATGTCCATTCTTCGGGTGTCAT-3'. 24 hours after the last injection, mice were sacrificed. 


\subsection{Isolation of hepatic cells}

Human hepatocytes and hepatic stellate cells (hHSC) were isolated from non-neoplastic tissue obtained from explanted or resected livers of 17 patients. All samples were negative for hepatitis B virus surface antigen (HBsAg) and for hepatitis C virus (HCV)-RNA. Samples from all-aged donors with variable steatosis and fibrosis grade were included in the study. Informed written consent of each patient were obtained, according to legal and ethical rules established by the Ethics Committee of the Fondazione IRCCS Cà Granda, which has already approved the protocol. Liver tissue were stored in a Celsior preservation solution for a maximum of 8 hours from the explantation, at $4^{\circ} \mathrm{C}$.

HSCs and hepatocytes were isolated by a multi-step ethylene glycol tetra-acetic acid (EGTA)/collagenase perfusion technique, through adequate microcannulation of small blood vessels to ensure efficient perfusion of tissue sample, as previously described (88).

-perfusion solution 1, based on Hanks solution (Life Technologies, Carlsbad, CA) and EGTA 0,5 mM (Sigma-Aldrich, St Louis, MO);

-perfusion solution 2, made of Hanks solution with collagenase type IV Sigma-Aldrich, St Louis, MO) 0,25 mg/ml, CaCl2 2 mM, HEPES 20 mM (Sigma-Aldrich, St Louis, MO).

All perfusion solutions were incubated at $37^{\circ} \mathrm{C}$ before use. Furthermore, we performed an 'in vitro' digestion in a Dnase type I (Roche, Basel Switzerland) solution, composed of Dnase type I 0,2 mg/ml, $\mathrm{CaCl} 22 \mathrm{mM}$ and $1 \%$ Pen/Strep.

Then, we separated HSCs, taking advantage of HSCs low density, due to a large amount of lipid droplets, since these cells store retinol as retinyl palmitate. For this purpose, we used a discontinuous density gradient of 15\% and 11,5\% Optiprep (Sigma-Aldrich, St Louis, MO), prepared according to the manufacturer's instruction. 
Cells were cultured on plastic in DMEM medium supplemented with $10 \%$ FBS, 2 mM Lglutamine, 100 units $/ \mathrm{ml}$ penicillin and $0.1 \mathrm{mg} / \mathrm{ml}$ streptomycin; $100 \mathrm{nM}$ insulin and $100 \mathrm{nM}$ dexamethasone were added for hepatocytes. Isolated cells' vitality was evaluated using flow cytometry and was around 99.5\%. At least, three independent lots of freshly isolated cells were used for experiments.

\subsection{Isolation of leukocytes from peripheral blood}

Peripheral blood lymphocytes and monocytes were isolated from 16 healthy control subjects with normal coagulation and iron metabolism parameters and without cardiovascular disease. They were healthy volunteers, whose age was comprised between 20 and 40 years, with serum ferritin (50$150 \mathrm{ng} / \mathrm{ml}$ ) and transferrin saturation (25-45\%) within the normal range, and without a previous history of cardiovascular disease. Written informed consent was obtained by each subject included in the study, which was approved by the Institutional Review Board of the Fondazione IRCCS Ca' Granda.

Mononuclear cells (monocytes and lymphocytes) were separated using the Lymphoprep gradient, because of their lower density (below $1.077 \mathrm{~g} / \mathrm{ml}$ ) compared to the erythrocytes and the polymorphonuclear (PMN) leukocytes (granulocytes). Therefore, these cells can be isolated starting from $200 \mathrm{ml}$ of peripheral blood anticoagulated with citrate $3.2 \%$ (89) by centrifugation on an isoosmotic medium with a density of $1.077 \mathrm{~g} / \mathrm{ml}$, which allows the erythrocytes and the PMNs to sediment through the medium while retaining the mononuclear cells at the sample/medium interface.

Monocytes and lymphocytes isolated were cultured in RPMI 1640 medium supplemented with 10\% FBS, 1\% $\beta$-Mercaptoethanol and 1\% Glutamine. After 24 hours, lymphocytes in media and monocytes attached were separated. In other experimental settings, M1 or M2 polarization of macrophages is inducted, treating monocytes with GM-CSF or MCSF (25 ng/ml) for 6 days. 


\subsection{In vitro treatments}

When specified, primary hepatocytes isolated from wild-type mice were pretreated for 1 hour with $50 \mu \mathrm{M}$ PI3K inhibitor (LY294002 - Cell Signaling Technologies, Boston, United States) or in other experimental settings with $50 \mathrm{nM}$ mTOR inhibitor (Rapamycin - Cell Signaling Technologies, Boston, United States), and then exposed to $0.33 \mu \mathrm{M}$ insulin (Insuman Rapid, Sanofi Aventis) for 6 hours.

\subsection{Patients}

We evaluated hepatic and adipose tissue samples of 119 unselected severely obese individuals (Bariatric surgery cohort), who underwent percutaneous liver biopsy performed during bariatric surgery for staging of liver damage severity. They included 23 obese patients without clinical and biochemical evidence of liver and metabolic disease and no history of alcohol abuse (men, $>30 \mathrm{~g} / \mathrm{d}$; women, $>20$ $\mathrm{g} / \mathrm{d}$ ) (referred to as normal liver group), 55 obese patients with steatosis (simple steatosis group) and 41 obese subjects whose liver biopsies fulfilled the histological criteria for NASH (NASH group). NASH was considered to be present when a) steatosis, b) lobular inflammation and c) hepatocellular ballooning were concomitantly present. The inclusion criteria were liver biopsy for severe obesity, availability of RNA samples and clinical data. Individuals with increased alcohol intake, viral and autoimmune hepatitis, hereditary haemochromatosis and alpha1-antitrypsin deficiency or other causes of liver disease were excluded. Informed written consent was obtained from each patient and the study protocol was approved by the Ethical Committee of the Fondazione IRCCS Ca' Granda, Milan and conforms to the ethical guidelines of the 1975 Declaration of Helsinki. The clinical characteristics of these patients are listed in Table $\mathbf{1}$. 
Table 1. Clinical characteristics of 119 severely obese patients (Bariatric surgery cohort), of whom MBOAT7 hepatic and adipose tissue expression was evaluated.

\begin{tabular}{|c|c|c|c|c|}
\hline & $\begin{array}{l}\text { Normal liver } \\
\qquad(\mathbf{n}=23)\end{array}$ & $\begin{array}{c}\text { Simple } \\
\text { steatosis } \\
(n=55)\end{array}$ & $\begin{array}{l}\text { NASH } \\
(n=41)\end{array}$ & Pvalue \\
\hline Age, years & $44 \pm 2$ & $42 \pm 2$ & $46 \pm 1.5$ & 0.30 \\
\hline Gender, F (\%) & $22(96)$ & $37(90)$ & $42(76)$ & 0.04 \\
\hline BMI, $\mathrm{Kg} / \mathrm{m} 2$ & $38 \pm 10$ & $41 \pm 5$ & $44 \pm 8$ & 0.0007 \\
\hline IFG/T2DM, yes $(\%)$ & $4(17)$ & $6(15)$ & $13(24)$ & 0.52 \\
\hline Total cholesterol, mg/dL & $195 \pm 56$ & $219 \pm 38$ & $207 \pm 49$ & 0.23 \\
\hline LDL cholesterol, mg/dL & $136 \pm 34$ & $136 \pm 29$ & $126 \pm 40$ & 0.36 \\
\hline HDL cholesterol, mg/dL & $62 \pm 11$ & $58 \pm 13$ & $48 \pm 16$ & 0.0012 \\
\hline Triglycerides, mg/dL & $119 \pm 42$ & $139 \pm 76$ & $159 \pm 81$ & 0.18 \\
\hline ALT, IU/1 & $14[11-21]$ & $20[14-24]$ & $28[20-44]$ & 0.0001 \\
\hline AST, IU/l & $15[12-18]$ & $18[16-20]$ & $22[18-27]$ & 0.0005 \\
\hline PNPLA3, I148M alleles yes (\%) & $12(53)$ & $21(51)$ & $37(67)$ & 0.2 \\
\hline MBOAT7, T alleles yes $(\%)$ & $12(53)$ & $30(73)$ & $40(73)$ & 0.2 \\
\hline
\end{tabular}

Values are mean \pm standard deviation $(\mathrm{SD})$, median [interquartile range], or number (\%). BMI: body mass index; IFG: impaired fasting glucose (defined as fasting glucose >110 mg/dL); T2DM: type 2 diabetes mellitus. 
We evaluated also serum free fatty acids (FFAs) in 72 lean NAFLD patients (Hepatology service cohort), stratified by the presence of MBOAT7 T allele of risk, of whom serum samples were available. Clinical characteristics of NAFLD patients are listed in Table 2.

Table 2. Clinical characteristics of 72 NAFLD patients (Hepatology service cohort), of whom serum FFAs concentration was evaluated.

\begin{tabular}{lc}
\hline & $\begin{array}{c}\text { Hepatology } \\
\text { service cohort (n=72) }\end{array}$ \\
\hline Age, years & $47 \pm 11$ \\
Gender, F (\%) & $8(11)$ \\
BMI, Kg/m2 & $26 \pm 3$ \\
IFG/T2DM, yes (\%) & $7(9.7)$ \\
Total cholesterol, mg/dL & $205 \pm 39$ \\
LDL cholesterol, mg/dL & $127 \pm 37$ \\
HDL cholesterol, mg/dL & $45 \pm 11$ \\
Triglycerides, mg/dL & $147 \pm 78$ \\
ALT, IU/l & $50[18-75]$ \\
AST, IU/l & $28[15-38]$ \\
PNPLA3, I148M alleles yes (\%) & $52(72)$ \\
MBOAT7, T alleles yes (\%) & $46(64)$ \\
\hline
\end{tabular}

Values are mean \pm standard deviation (SD), median [interquartile range], or number (\%). BMI: body mass index; IFG: impaired fasting glucose (defined as fasting glucose $>110 \mathrm{mg} / \mathrm{dL}$ ); T2DM: type 2 diabetes mellitus. 
All patients were previously genotyped for rs738409 (PNPLA3 I148M) and rs641738 (MBOAT7/TMC4) using TaqMan 5'-nuclease assays (Life Technologies, Carlsbad, CA), as previously described (51)

\subsection{Gene expression analysis}

RNA was extracted from tissues or cell cultures using Trizol reagent (Life TechnologiesThermofisher Scientific, Carlsbad, U.S.A). $1 \mu \mathrm{g}$ of total RNA was retro-transcribed with VILO random hexamers synthesis system (Life Technologies-Thermofisher Scientific, Carlsbad, U.S.A). Quantitative real time PCR (qRT-PCR) was performed by a ABI 7500 fast thermocycler (Life Technologies), using the Taqman Universal PCR Master Mix (Life Technologies, Carlsbad, CA) and Taqman probes for human MBOAT7 and the SYBR Green chemistry (Fast SYBR Green Master Mix; Life Technologies) for all other genes. All reactions were delivered in triplicate. Data were normalized to the $\beta$-actin gene expression and results were expressed as arbitrary units or fold increase as indicated in bar graphs. Primers are listed in Table 3.

Table 3: Quantitative PCR analysis primer sequences

\begin{tabular}{|c|c|c|}
\hline & Sense & Antisense \\
\hline ApoB100 & AAGCACCTCCGAAAGTACGTG & CTCCAGCTCTACCTTACAGTTGA \\
\hline Fabp1 & TCATGAAGGCAATAGGTCTGC & TTGACGACTGCCTTGACTTT \\
\hline Fatp1 & CGCTTTCTGCGTATCGTCTG & GATGCACGGGATCGTGTCT \\
\hline FATP1 Hs & CCACTTGGATGTCACCACTG & GTGGGACCCTCCAGTAGACA \\
\hline
\end{tabular}




\begin{tabular}{|c|c|c|}
\hline Fasn & AAGGCTGGGCTCTATGGATT & TGAGGCTGGGTTGATACCTC \\
\hline FASn Hs & GCTGGGTGGAGTCTCTGAAG & TGCAACACCTTCTGCAGTTC \\
\hline Cpt1 & CCAGGCTACAGTGGGACATT & GAACTTGCCCATGTCCTTGT \\
\hline Cxcl10 & AGTGGGACTCAAGGGATCC & GTCAGGCCAACAGAGCTGAT \\
\hline Mboat5 & GGCCTCTCAATTGCTTATTTCA & AGCACGACACATAGCAAGGA \\
\hline Mboat7 & AAGAAAGCTGGACCTGGGC & CTTCAGTGTCAACAGCAGCT \\
\hline Mttp & GCTTCCGTTAAAGGTCACACA & CAGCTGTTATCGTGACTTGGA \\
\hline Ppar-a & ATGCCAGTACTGCCGTTTTC & GGCCTTGACCTTGTTCATGT \\
\hline Srebp-1c & GATCAAAGAGGAGCCAGTGC & TAGATGGTGGCTGCTGAGTG \\
\hline SREBP1 Hs & TGCATTTTCTGACACGCTTC & CCAAGCTGTACAGGCTCTCC \\
\hline Tmc4 & TTGACACTACAGGAGACGCC & TTCTGCCTCCATGTTTCCCC \\
\hline Tnf-a & CCCCAAAGGGATGAGAAGTT & GTGGGTGAGGAGCACGTAGT \\
\hline$\beta$-actin & GCTACAGCTTCACCACCACA & AAGGAAGGCTGGAAAAGAGC \\
\hline
\end{tabular}

\subsection{Western blot analysis}

Total protein lysates were extracted from $20 \mathrm{mg}$ of tissue or from cell cultures, using RIPA buffer containing $1 \mathrm{mmol} / \mathrm{L} \mathrm{Na-orthovanadate,} 200 \mathrm{mmol} / \mathrm{L}$ phenylmethyl sulfonyl fluoride and 0.02 $\mu \mathrm{g} / \mu \mathrm{L}$ aprotinin. Equal amounts of proteins $(50 \mu \mathrm{g})$ were separated by SDS-PAGE, transferred electrophoretically to nitrocellulose membrane (BioRad, Hercules, CA) and incubated with specific antibodies. Antibodies and concentration used are listed in Table 4. FoxA2 protein expression was assessed by western blot also in cytosolic and nuclear protein fraction in mice fasted 16 hours and then 
refed for 15 minutes, 1 hour, 4 hours and 8 hours, in order to study the localization of this transcription factor in consequence to the rise of insulin signaling. The separation and preparation of cytoplasmic and nuclear extract from tissue and cell lines was performed using NE-PER Nuclear and Cytoplasmic extraction reagents (Thermo Scientific, Carlsbad, U.S.A).

Table 4. Antibodies and dilution used in Western blotting and immunohistochemistry.

\begin{tabular}{cc}
\hline Antibody & Catalog Number \\
Thr308 Akt (1:1000) & Cell signaling \#2965 \\
Akt (1:1000) & Cell signaling \#2938 \\
FoxO1 (1:1000) & Cell signaling \#2880 \\
Foxa2 (1:1000) & Abcam ab23630 \\
Histone H3 (1:2000) & Abcam ab1791 \\
Mboat7 (1:500) & Sigma-Aldrich \#AV49811 \\
P(T389)P70S6 Kinase (1:1000) & Cell signaling \#9205 \\
P70S6 Kinase (1:1000) & Cell signaling \#9202 \\
B-actin (1:1000) & Santa-Cruz sc-1615 \\
\hline
\end{tabular}

\subsection{Statistical Analysis}

Results are shown as means \pm SD. Statistical analysis was performed using JMP 12.0 (SAS, Cary, NC) by using two-way analysis of variance (ANOVA) or chi-square test, where appropriate. Independent determinants of MBOAT7 down-regulation in liver and adipose tissue of severely obese 
patients were assessed by multivariate generalized linear model analysis, adjusted for the confounders specified in the Results section. Determinants of FFAs increase in serum samples from lean NAFLD patients were identified by multivariate generalized linear model analysis. Correlations with FATP1 increased expression in liver of severely obese patients were assessed by bivariate analysis. $\mathrm{P}$ values $<0.05$ (two-tailed) were considered significant. 


\section{Results}

\subsection{MBOAT7 down-regulation is associated with NAFLD severity independently of rs641737 $\mathrm{C}>\mathrm{T}$ genotype}

To investigate whether the blunted expression of MBOAT7 may affect the liver damage severity independently of the presence of MBOAT7 $\mathrm{T}$ allele, we evaluated the hepatic MBOAT7 expression in 119 Italian severely obese patients at risk of NASH, who underwent to liver biopsy during bariatric surgery (Bariatric surgery cohort). Demographic and clinical features are shown in Table 1. The main finding was that hepatic MBOAT7 mRNA levels progressively decreased from normal liver to simple steatosis and NASH ( $<<0.05 v s$. normal liver; Fig. 6A).

In particular, at multivariate generalized linear model, impaired fasting glucose/type 2 diabetes mellitus (IFG/T2DM; estimate $-0.16 \pm 0.08 ; \mathrm{p}=0.04$ ), necroinflammation (estimate $-0.31 \pm 0.11 ; \mathrm{p}=0.005$ ) and MBOAT7 risk allele (estimate $-0.25 \pm 0.08 ; \mathrm{p}=0.001$ ) were independently associated with reduced MBOAT7 expression (Table 5; Fig. 6B-C), independently of several confounders. These data suggest that the down-regulation of hepatic MBOAT7 is associated with development and severity of NAFLD independently of MBOAT7 genotype. 
Table 5: Variables associated with hepatic MBOAT7 gene expression at multivariate generalized linear model analysis, adjusted for all the clinical features reported in the table, in 119 severely obese patients who underwent to liver biopsy during bariatric surgery.

\begin{tabular}{lcc}
\hline & Estimate SE & P value \\
\hline Age, years & $-0.00 \pm 0.01$ & 0.98 \\
Sex, F & $-0.07 \pm 0.09$ & 0.43 \\
BMI, Kg/m ${ }^{2}$ & $+0.00 \pm 0.01$ & 0.87 \\
IFG/T2DM, yes & $-0.16 \pm 0.08$ & $\mathbf{0 . 0 4}$ \\
Steatosis & $-0.02 \pm 0.07$ & 0.84 \\
Necroinflammation & $-0.31 \pm 0.11$ & $\mathbf{0 . 0 0 5}$ \\
PNPLA3, I148M alleles & $-0.04 \pm 0.09$ & 0.66 \\
MBOAT7, T alleles & $-0.25 \pm 0.08$ & $\mathbf{0 . 0 0 4}$ \\
\hline
\end{tabular}

BMI: body mass index; IFG: impaired fasting glucose (defined as fasting glucose $>110 \mathrm{mg} / \mathrm{dL}$ ); T2DM: type 2 diabetes mellitus. 
A

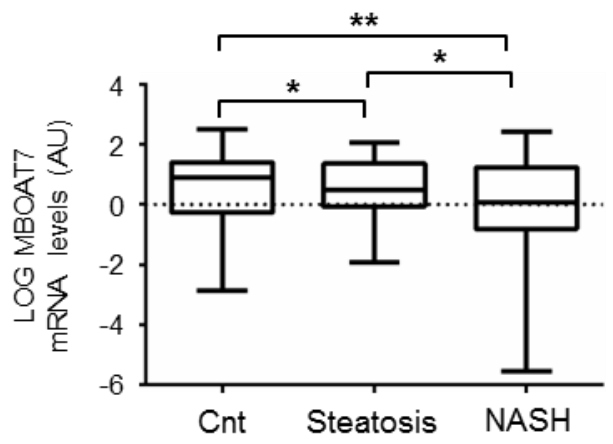

C

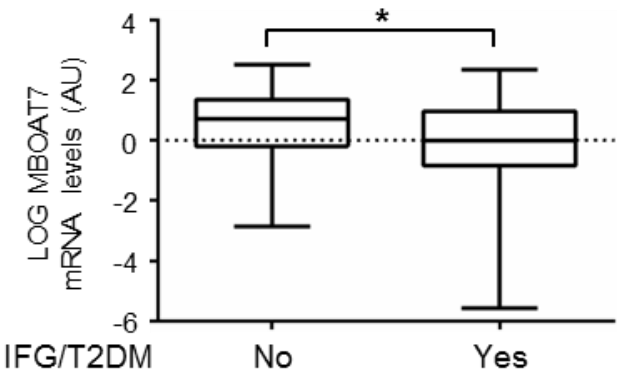

B

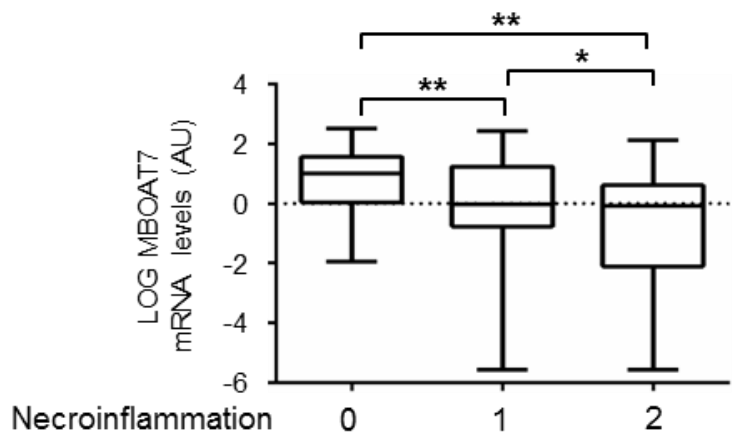

Fig. 6: Hepatic MBOAT7 expression decreases with liver damage severity. Hepatic MBOAT7 mRNA levels were evaluated by qRT-PCR in 119 severely obese patients stratified for liver damage severity (Normal liver; Control group (Cnt) n=23; Simple Steatosis $n=55$; NASH $n=41$ ) (A) for necroinflammatory grade (B) or for impaired fasting glucose/type 2 diabetes mellitus (IFG/T2DM) presence (C). Demographic, anthropometric and clinical characteristics of these patients are shown in Table S1. Data were normalized to $\beta$-actin and expressed as fold increase (Arbitrary Units - AU), compared to Cnt group. ${ }^{*} \mathrm{p}<0.05$, $* * \mathrm{p}<0.01$ compared to Cnt.

Furthermore, since the complex interplay between liver and adipose tissue in insulin resistance (IR) conditions is involved in the development and in the progression of NAFLD, we assessed also the adipose tissue expression of MBOAT7 in this cohort of obese patients. Notably, the expression of MBOAT7 in the adipose tissue was 9-fold lesser than in the liver. At multivariate generalized linear model analysis, IFG/T2DM (estimate $-0.28 \pm 0.12 ; \mathrm{p}=0.01$ ) and MBOAT7 risk allele (estimate $0.31 \pm 0.13 ; \mathrm{p}=0.02)$ were independently associated with MBOAT7 expression after adjustment for age, sex, BMI, presence of T2DM, the severity of liver disease, PNPLA3, I148M alleles and MBOAT7, T 
alleles, confirming the effect of IFG/T2DM and of the rs641738 variant on the down-regulation of MBOAT7 mRNA levels also in adipose tissue (Table 6).

Table 6: Variables associated with adipose MBOAT7 gene expression at multivariate generalized linear model analysis in 119 severely obese patients who underwent to adipose tissue biopsy during bariatric surgery.

\begin{tabular}{lcc}
\hline & Estimate SE & P value \\
\hline Age, years & $0.014 \pm 0.01$ & 0.15 \\
Sex, F & $-0.21 \pm 0.12$ & 0.09 \\
BMI, Kg/m ${ }^{2}$ & $0.00 \pm 0.02$ & 0.77 \\
IFG/T2DM, yes & $-0.28 \pm 0.12$ & $\mathbf{0 . 0 1}$ \\
Steatosis & $0.03 \pm 0.12$ & 0.78 \\
Necroinflammation & $0.07 \pm 0.17$ & 0.66 \\
PNPLA3, I148M alleles & $-0.28 \pm 0.16$ & 0.09 \\
MBOAT7, T alleles & $-0.31 \pm 0.13$ & $\mathbf{0 . 0 2}$ \\
\hline
\end{tabular}

BMI: body mass index; IFG: impaired fasting glucose (defined as fasting glucose >110 mg/dL); T2DM: type 2 diabetes mellitus.

Then, to dissect the impact of the rs641738 $\mathrm{C}>\mathrm{T}$ variant on specific hepatic cell types, we isolated hepatocytes and human hepatic stellate cells (hHSCs) from non-neoplastic tissue obtained from 
explanted or resected livers of 17 patients. MBOAT7 mRNA levels were similar between hepatocytes and hHSCs. However, the rs641738 variant was associated with reduced MBOAT7 expression only in hepatocytes (p<0.05 vs. CC; Fig. 7), indicating that the effect of MBOAT7 down-regulation on TG accumulation, previously described, may be a cell-autonomous property of hepatocytes.

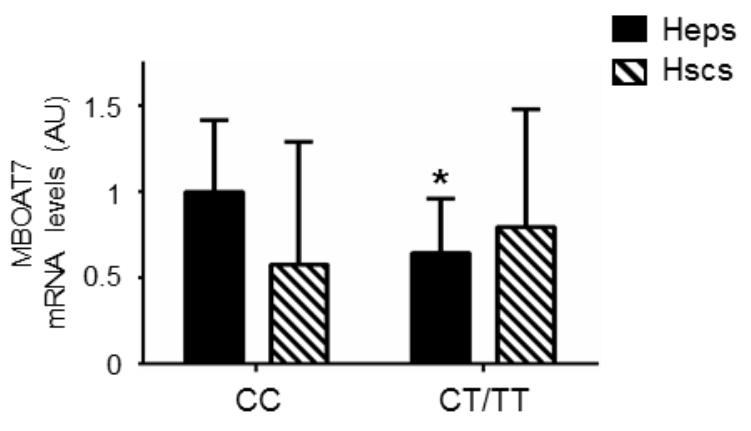

Fig. 7: MBOAT7 rs641738 C>T variant affects MBOAT7 expression in hepatocytes, but not in human hepatic stellate cells (hHSCs). MBOAT7 mRNA levels were evaluated by qRT-PCR in freshly human hepatocytes and activated hHSCs (at third passage of culture) isolated from patients wild-type (CC) or carriers of MBOAT7 T allele. mRNA levels were normalized for $\beta$-actin and expressed as fold increase (Arbitrary Units - AU), as compared to CC genotype. ( $\mathrm{n}=17$ hepatocytes; $\mathrm{n}=12 \mathrm{hHSCs}$ ) $* \mathrm{p}<0.05$ compared to protective $\mathrm{CC}$ genotype.

Finally, to gain insight into impact of MBOAT7 genotype in inflammatory cells, we isolated peripheral blood lymphocytes and monocytes from 16 healthy subjects. MBOAT7 gene expression tended to decrease both in lymphocytes and monocytes of patients carrying $\mathrm{T}$ allele of risk $(\mathrm{p}<0.05 v s$. $\mathrm{CC}$ and $\mathrm{p}=0.09$ vs. CC respectively; Fig. 8). MBOAT7 was highly expressed by human primary monocytes (7-fold more than in hepatocytes and HSCs). Interestingly, differentiation of monocytes with polarization to both $\mathrm{M} 1$ or M2 macrophages induced MBOAT7 reduction, independently of the presence of $\mathrm{T}$ allele ( $<0.05$ vs. monocytes; Fig. 8). These data are consistent with the hypothesis that MBOAT7 down-regulation may be involved in priming macrophages for triggering inflammation. 


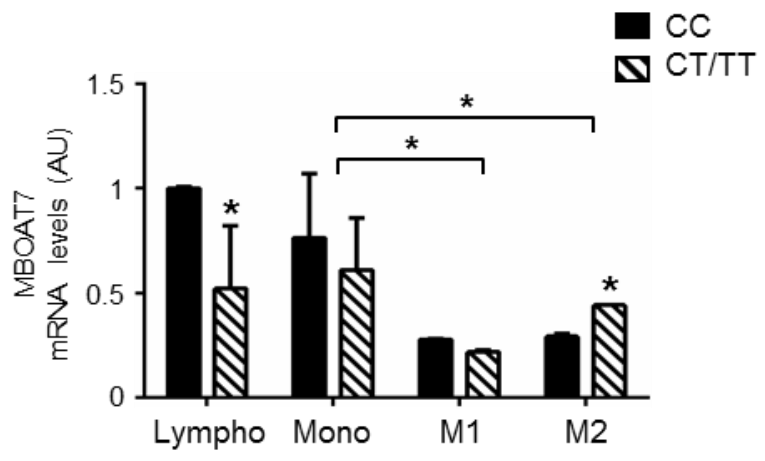

Fig. 8: MBOAT7 expression decreases with inflammatory cells activation. MBOAT7 mRNA levels were evaluated by qRT-PCR in freshly isolated human lymphocytes, monocytes and M1 or M2 macrophages, stratified for genotype. mRNA levels were normalized to $\beta$-actin and expressed as fold increase (Arbitrary Units - AU), compared to CC lymphocytes. ( $n=7$ lymphocytes; $n=15$ monocytes; $n=3$ M1 and M2) *p<0.05 compared to protective CC genotype.

\subsection{Mboat7 expression is dampened in Lep ${ }^{o b / o b}$ mice}

To dissect the contribution of different pathophysiological triggers in Mboat7 regulation, we next evaluated the hepatic Mboat7 expression in experimental mice models of NAFLD, such as methionine choline deficient diet (MCD), that induces NASH, but not IR, and in genetically obese Lep $^{o b / o b}$ mice which develop IR. As expected, hepatic TG content was higher in MCD-fed mice compared to mice fed standard diet (SD) ( $\mathrm{p}<0.05$; Fig. 9A-B), but more so in Lep ${ }^{o b / o b}$ mice $(\mathrm{p}<0.01 v s$. SD-fed mice). Fasting insulin was increased in Lep ${ }^{o b / o b}$ mice compared to wild-type mice fed SD or MCD ( $<<0.01$; Fig. 9C) and expectedly they showed higher glucose levels during fasting and following insulin tolerance test $(\mathrm{p}<0.05, \mathrm{p}<0.01$ respectively; Fig 9D, E), confirming the insulin resistance in Lep ${ }^{o b / o b}$ mice. In line with the results obtained in patients, hepatic Mboat7 mRNA and protein expression were reduced in MCD-fed mice ( $\mathrm{p}<0.05$ vs. SD-fed mice; Fig. 9F-G), but more so in Lep ${ }^{o b / o b}$ mice $(\mathrm{p}<0.01$ vs. SD-fed mice; Fig. 9F-G), in which NAFLD and IR coexist. These data 
suggest that more severe hepatic Mboat7 down-regulation in Lep ${ }^{o b / o b}$ mice as compared to wild-type mice fed MCD may be explained by the presence of IR.
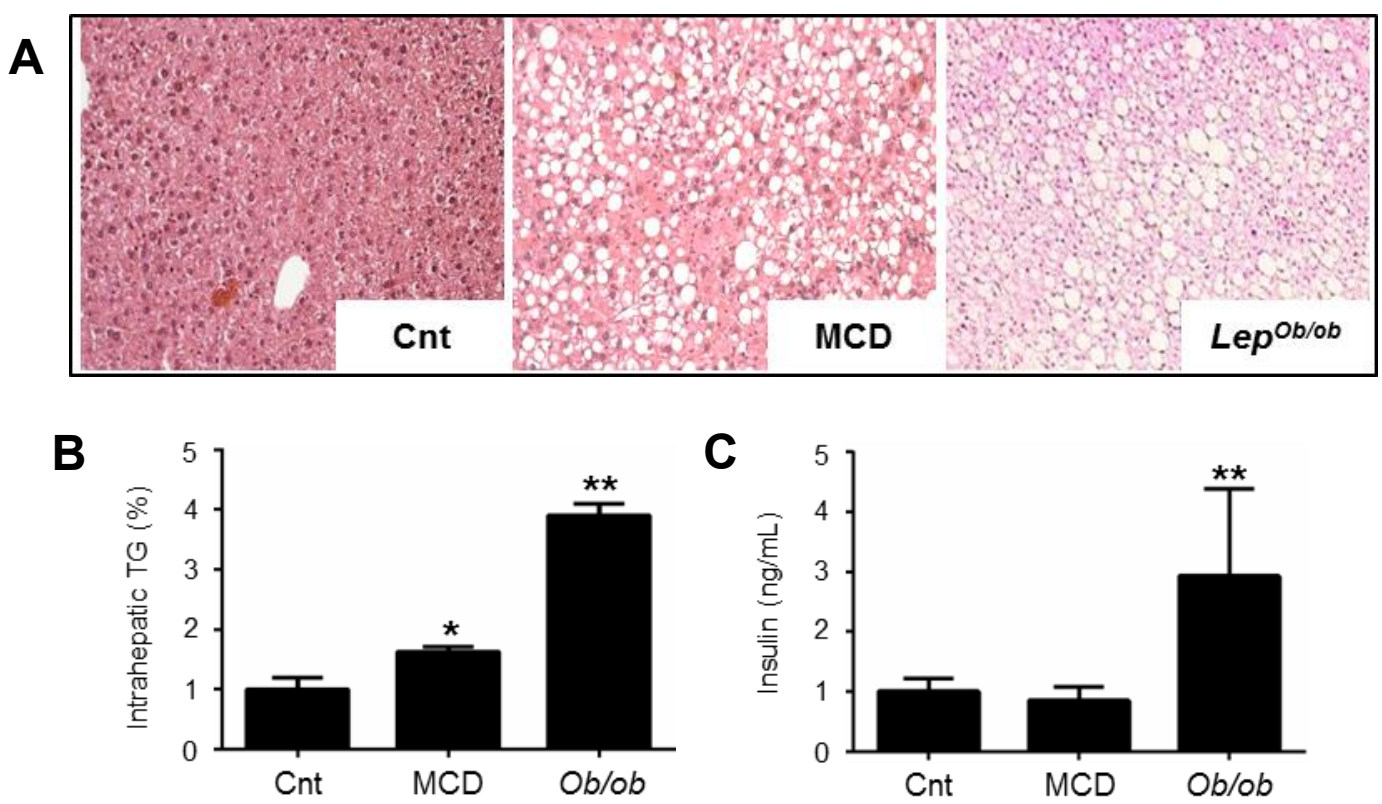

D

E
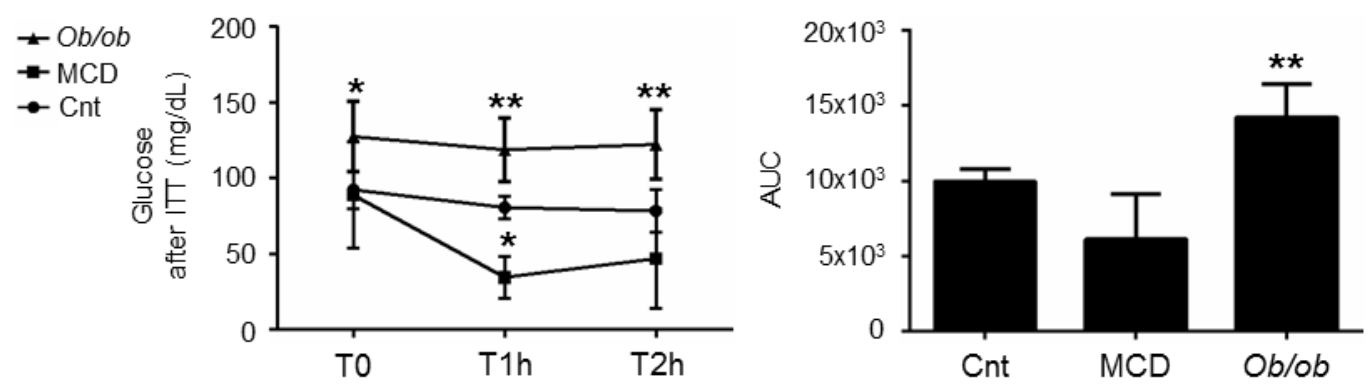

$\mathbf{F}$

G
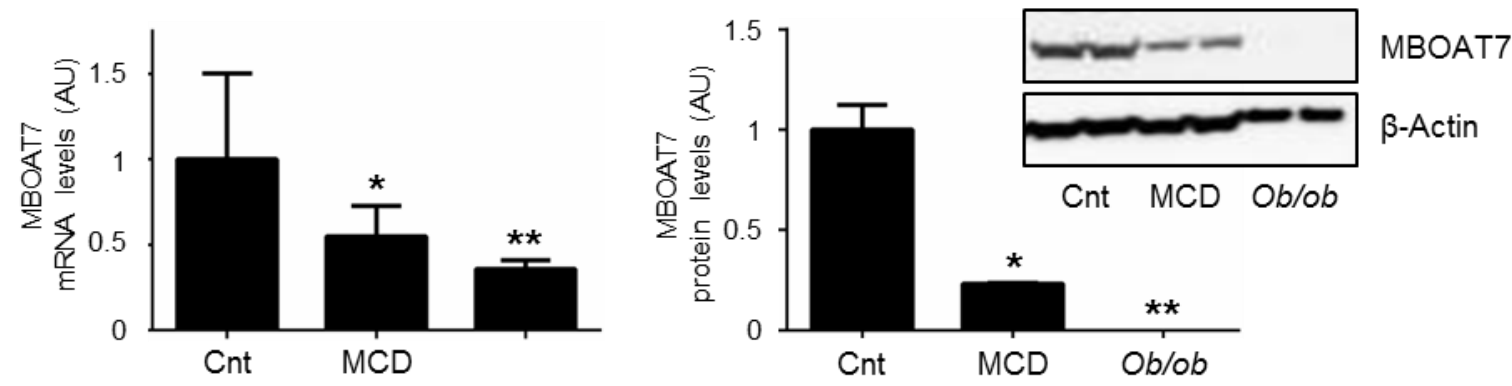

Fig. 9: Mboat7 expression decreases in experimental model of NASH. Hematoxylin and Eosin (H\&E) stain of hepatic specimens (A) and hepatic triglyceride (TG) content (B) from wild-type mice fed standard diet (referred to as Cnt) or Methionine choline deficient diet (MCD) and from Lep ${ }^{o b / o b}$ mice. Original magnification: $200 \mathrm{X}$. 
Serum insulin was measured in Cnt or MCD-fed wild-type mice and in Lep ${ }^{o b / o b}$ mice, fasted for 16 hours $(\mathrm{n}=10$ per group) (C). Insulin tolerant test (ITT, $0.75 \mathrm{U} / \mathrm{Kg}$ i.p). was performed in Lep $^{o b / o b}$ mice fasted for 16 hours. The circles refer to Cnt; squares refer to wild-type mice fed MCD and triangles to Lep ${ }^{o b / o b}$ mice. 10 mice per group were analyzed. Data are expressed as means $\pm \mathrm{SD} .{ }^{*} \mathrm{p}<0.05, * * \mathrm{p}<0.01$ compared to Cnt mice at basal condition, before insulin administration (T0) (D). Area under the curve (AUC) after ITT in Cnt, MCD and Lep $^{o b / o b}$ mice $(* * \mathrm{p}<0.01)(\mathbf{E})$. Hepatic Mboat7 mRNA levels were evaluated by qRT-PCR. mRNA levels were normalized for $\beta$-actin and expressed as fold increase (Arbitrary Units - AU), as compared to Cnt (F). Western blot and quantification of Mboat7 protein levels in livers from Cnt, MCD and Lep ${ }^{o b / o b}$ mice, fasted for 16 hours $\left(\mathrm{n}=10\right.$ per group) $(\mathbf{G}) .{ }^{*} \mathrm{p}<0.05, * * \mathrm{p}<0.01$ compared to $\mathrm{Cnt}$.

\subsection{InsR+/- mice show blunted Mboat7 expression, independently of diet}

To evaluate the independent contribution of hepatic fat accumulation, IR, and inflammation, we managed to dissociate these components, that are usually intertwined in patients, in an experimental model of NASH. To this end, we studied Mboat7 regulation in Insulin receptor haplo-insufficient mice (InsR+/-), characterized by development of hyperinsulinemia, fed standard or MCD diet for 6 weeks. InsR+/- mice developed more severe steatosis compared to wild-type MCD-fed mice (Fig. 10A) (36). Mboat7 mRNA levels were reduced in InsR+/- compared to wild-type mice, independently of the diet $(\mathrm{p}<0.05$; Fig. 10B). However, Mboat7 protein levels in InsR+/- mice fed-SD only slightly decreased compared to wild-type mice fed-SD ( $\mathrm{p}=0.1$; Fig. 10C). These data suggest that altered insulin signaling may play a causal role in determining Mboat7 down-regulation. The presence of inflammation, induced by MCD, did not further reduce Mboat7 expression in InsR+/- mice compared to wild-type littermates feeding the same diet, probably because of the impaired activation of hepatic leukocytes and the lower release of pro-inflammatory cytokines, identified in InsR+/- mice (data not shown). 

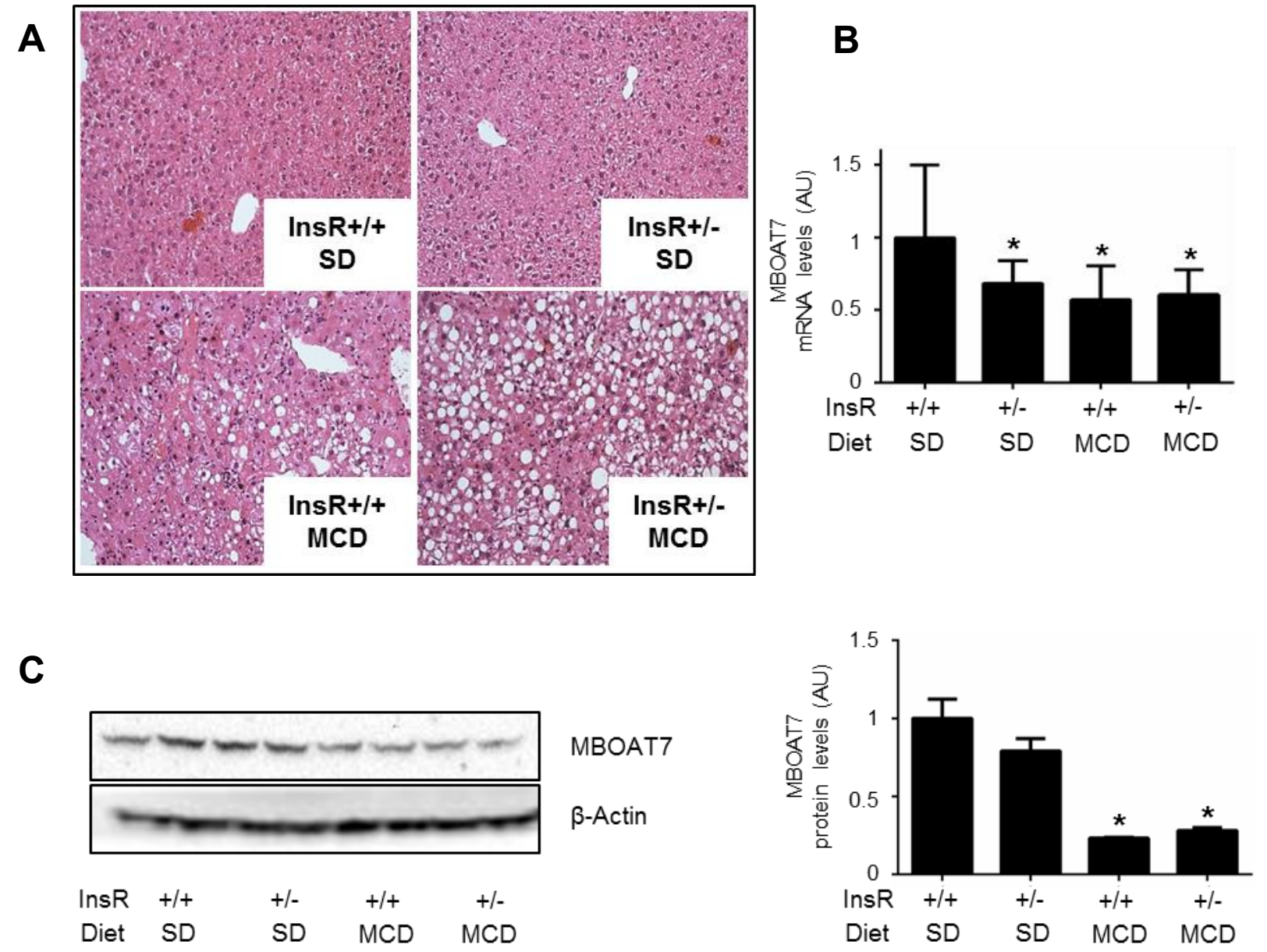

Fig. 10: Insulin receptor haplo-insufficient (InsR+/-) mice show decreased Mboat7 expression, independently of diet. H\&E stain of hepatic specimens from wild-type and InsR+/- mice fed standard diet (SD) or MCD (A). Original magnification: 200X. Hepatic Mboat7 mRNA levels were evaluated by qRT-PCR. mRNA levels were normalized for $\beta$-actin and expressed as fold increase (Arbitrary Units - AU), as compared to wild-type SD $($ InsR+/+ SD) (B). Western blot and quantification of Mboat7 protein levels in livers from wild-type and InsR+/MCD mice, fasted for 16 hours ( $\mathrm{n}=10$ per group) $(\mathbf{C}) . * \mathrm{p}<0.05, * * \mathrm{p}<0.01$ compared to InsR+/+ SD.

Then, to test whether down-regulation of Mboat7 in response to IR may be generalizable to other models of NAFLD, we evaluated its expression also in InsR+/- or wild-type mice fed MCD supplemented with fructose, a major substrate for hepatic lipogenesis. Additional fructose supplementation was associated with more severe steatosis (data not shown), but did not further 
decrease hepatic Mboat7 mRNA levels in InsR+/- mice fed MCD compared to InsR+/- mice fed SD (Fig. 11). These data suggest that IR, but not hepatic lipid accumulation alone, specifically induces Mboat7 down-regulation.

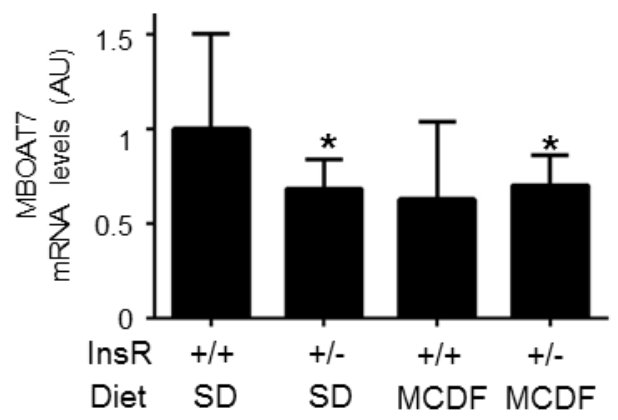

Fig. 11: Fructose supplementation to MCD does not exacerbate Mboat7 down-regulation in InsR+/- mice. Hepatic Mboat7 mRNA levels were evaluated by qRT-PCR in InsR+/- and wild-type mice fed MCD supplemented with $30 \%$ fructose. mRNA levels were normalized to $\beta$-actin and expressed as fold increase (Arbitrary Units - AU), compared to wild-type SD-fed mice (InsR+/+ SD).

\subsection{Hepatic Mboat7 expression depends on nutritional state}

To examine whether Mboat7 is physiologically regulated by excursions in insulin levels following fasting-refeeding cycles, we analyzed its expression in male mice after overnight fasting and then after a time course of refeeding (15 minutes, 1 hour, 4 hours, 8 hours). Biochemical features of wild-type male mice fasted for 16 hours or refed are shown in Table 7. 
Table 7. Biochemical features of wild-type male mice fasted for 16 hours or refed (15 min, 1 hour, 4 hours or 8 hours).

\begin{tabular}{lcccccc}
\hline & T0 & T15 & T1h & T4h & T8h & $\begin{array}{c}\boldsymbol{P} \\
\text { value }\end{array}$ \\
\hline Liver/Body Weight & $0.04 \pm 0.002$ & $0.05 \pm 0.002$ & $0.05 \pm 0.002$ & $0.053 \pm 0.003$ & $0.06 \pm 0.002$ & $\mathbf{0 . 0 2}$ \\
$\begin{array}{l}\text { Glucose (mg/dL) } \\
\text { Serum Triglycerides }\end{array}$ & $101 \pm 17.1$ & $108 \pm 14.6$ & $154 \pm 18.4$ & $116 \pm 19.4$ & $104 \pm 15$ & $\mathbf{0 . 0 4}$ \\
$\begin{array}{l}\text { (mg/dL) } \\
\text { Hepatic Triglycerides }\end{array}$ & $7.4 \pm 2$ & $4.6 \pm 2.1$ & $4 \pm 2.3$ & $3.6 \pm 2.9$ & $2.6 \pm 1.6$ & $\mathbf{0 . 0 2}$ \\
$\begin{array}{l}\text { (\%) } \\
\text { Cholesterol (mg/dL) }\end{array}$ & $99.7 \pm 23.1$ & $90.2 \pm 7.5$ & $81.4 \pm 24.2$ & $102.6 \pm 15.1$ & $101.7 \pm 15.3$ & 0.2 \\
ALT (mg/dL) & $44[40-50]$ & $52[48-60]$ & $60[48-64]$ & $72[62-102]$ & $40[36-72]$ & 0.4 \\
\hline
\end{tabular}

Values are expressed as mean \pm standard deviation (SD) or median [interquartile range]. N=7 mice/group.

Concomitantly with the rise of insulin levels ( $\mathrm{p}<0.05$ vs. fasting (T0); Fig. 12A) and the activation of hepatic insulin signaling, supported by an increased phospho-Akt/Akt ratio, the main intracellular mediator of insulin signaling activation, ( $\mathrm{p}<0.05$ vs. T0; Fig. 12A) and by a decreased Forkhead box protein O1 (FoxO1), the key transcription factor of gluconeogenesis, (p<0.05 vs. T0; Fig. 12A), both hepatic mRNA and protein levels of Mboat7 were down-regulated by refeeding ( $\mathrm{p}<0.05$ vs. T0; Fig. 12B). Consistently with these results, Mboat7 mRNA levels were also down-regulated in proximal intestine and in adipose tissue after refeeding ( $\mathrm{p}<0.05$ vs. T0; Fig. 12C-D), confirming the ability of insulin signaling activation to induce Mboat7 down-regulation also in other tissues. 
A

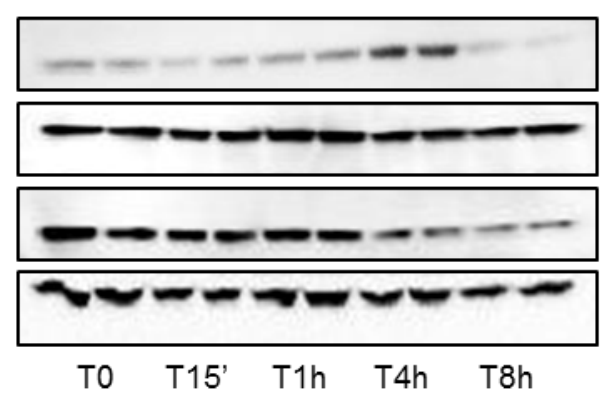

B

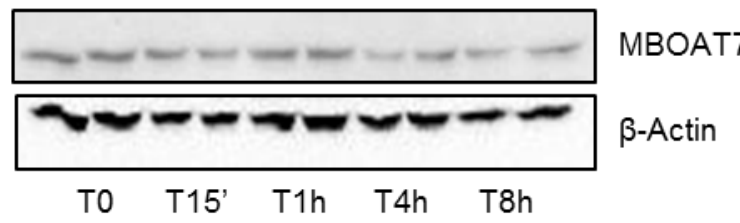

C

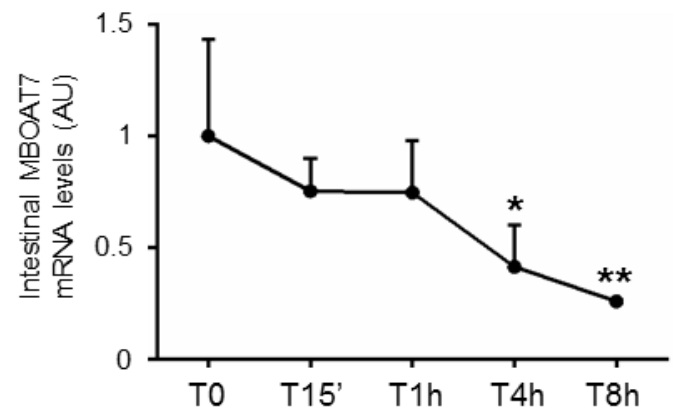

P-AKT

AKT

FOX01

$\beta$-Actin
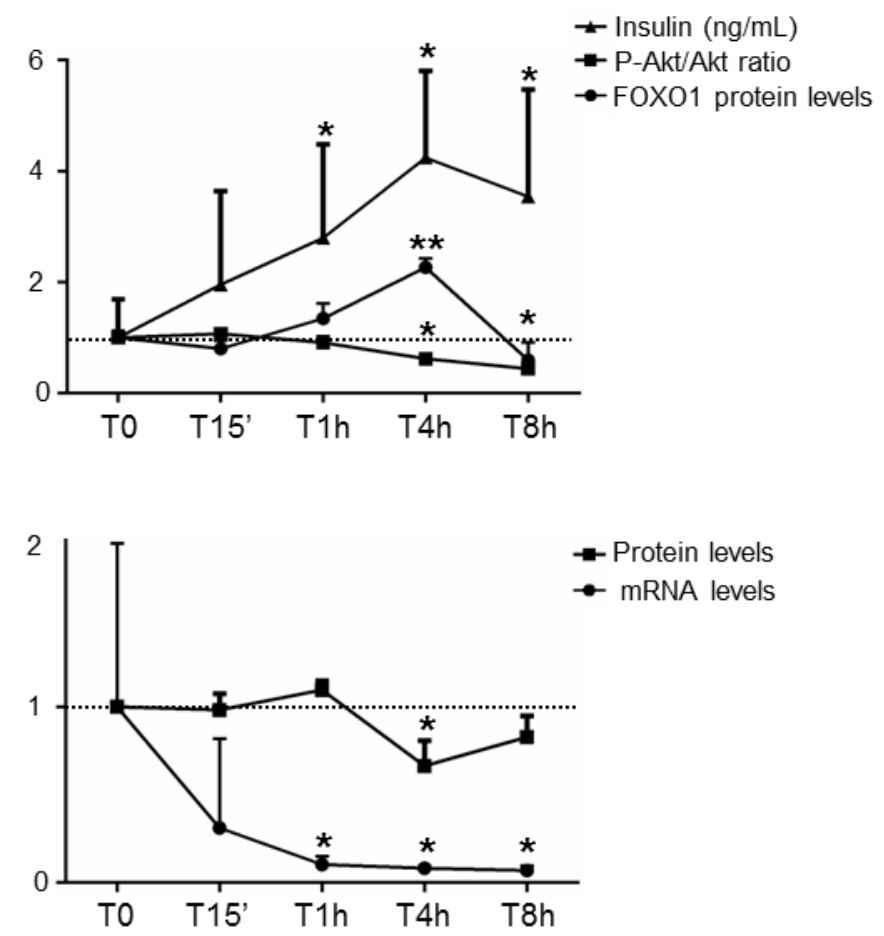

D

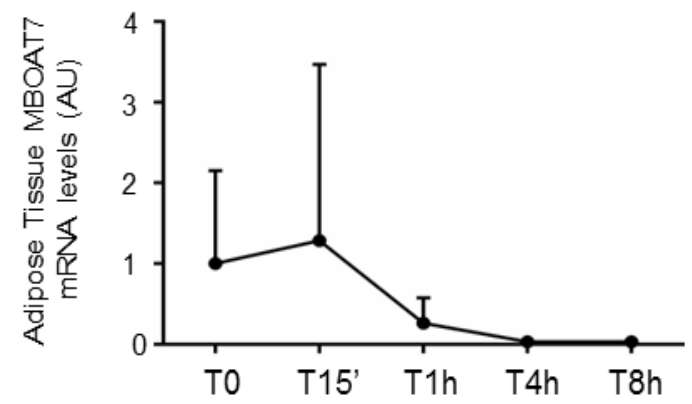

Fig. 12: Hepatic Mboat7 expression depends on nutritional state. Western blot and quantification of hepatic pThr308-Akt/Akt ratio, FoxO1 (A) and Mboat7 (B) protein levels and serum insulin evaluated in wild-type mice, fasted for 16 hours (T0) or refed for 15 minutes (T15'), 1 hour (T1h), 4 hours (T4h) or 8 hours (T8h) (n=7 per group). Hepatic (B), intestinal (C) and adipose tissue (D) Mboat7 mRNA levels were evaluated by qRTPCR. mRNA levels were normalized for $\beta$-actin and expressed as fold increase (Arbitrary Units - AU), as compared to T0. ${ }^{*} \mathrm{p}<0.05, * * \mathrm{p}<0.01$ compared to T0.

Since MBOAT5, another member of MBOAT family, have a similar ability to MBOAT7 to catalyze the incorporation of free arachidonic acids preferentially into the phosphatidylcholine (PC) in 
order to promote unsaturation of membrane lipids and lipoproteins production, and loss-of-function mutation in Mboat5 have been recently associated with an increased risk of hepatic steatosis onset, we sought to evaluate whether Mboat5 expression could also be influenced by insulin peak, due to the refeeding state. We found that Mboat5 mRNA levels were decreased after refeeding in liver and proximal intestine, where it is most abundant $(\mathrm{p}<0.05$ vs. T0; Fig. 13A-B). However, during fasting hyperinsulinemia associated with NAFLD development Mboat7 and Mboat5 showed a discordant regulation. Indeed, Lep ${ }^{o b / o b}$ and InsR+/- MCD-fed mice displayed enhanced hepatic expression of Mboat5 (p<0.05 vs. SD-fed mice; Fig. 12C-D).

A

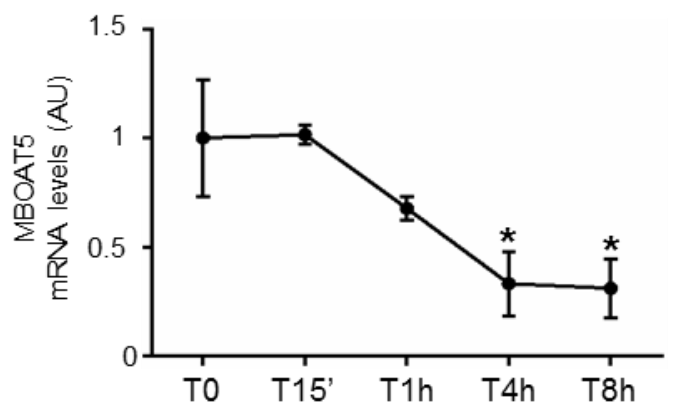

C

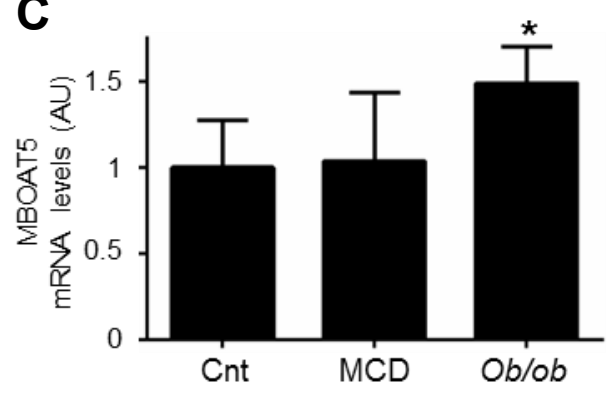

B

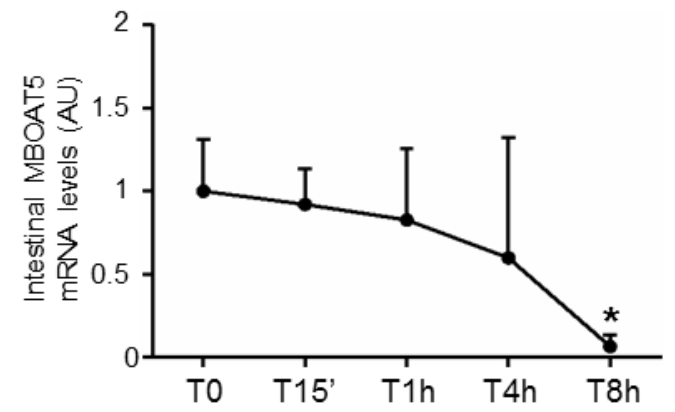

D

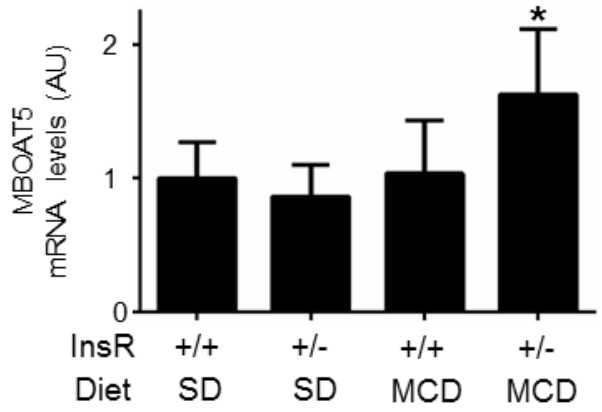

Fig. 13: Mboat5 increases in insulin resistant mice. Hepatic (A) and intestinal (B) Mboat5 mRNA levels were evaluated by qRT-PCR, normalized to $\beta$-actin and expressed as fold increase (Arbitrary Units - AU), compared to fasting condition (T0). ${ }^{*} \mathrm{p}<0.05$ compared to T0. Hepatic Mboat5 mRNA levels were evaluated also in wildtype and InsR+/- mice fed standard diet (Cnt) or MCD and in Lep ${ }^{o b / o b}$ mice $(\mathbf{C}, \mathbf{D})$. All data are expressed as fold increase (Arbitrary Units - AU), compared to Cnt. 


\subsection{Mboat7 down-regulation is dependent on insulin signaling activation in primary hepatocytes}

To test whether Mboat7 down-regulation may be insulin-dependent, we isolated primary hepatocytes from wild-type male mice and we treated them with insulin $(0.33 \mu \mathrm{M})$ alone or in combination with a selective inhibitor of insulin signaling activation (LY294002) which specifically acts on PI3Kinase, the kinase upstream to Akt. In keeping with the data obtained from the in vivo models, insulin addition to the culture media forced around 50\% of Mboat7 protein levels reduction ( $<<0.01$ vs. untreated hepatocytes; Fig. 14). Moreover, the effect of insulin on Mboat7 dampening was completely abolished by the pharmacological inhibition of the signaling pathway by the PI3Kinase inhibitor ( $<<0.01$ vs. insulin-treated hepatocytes; Fig. 14).
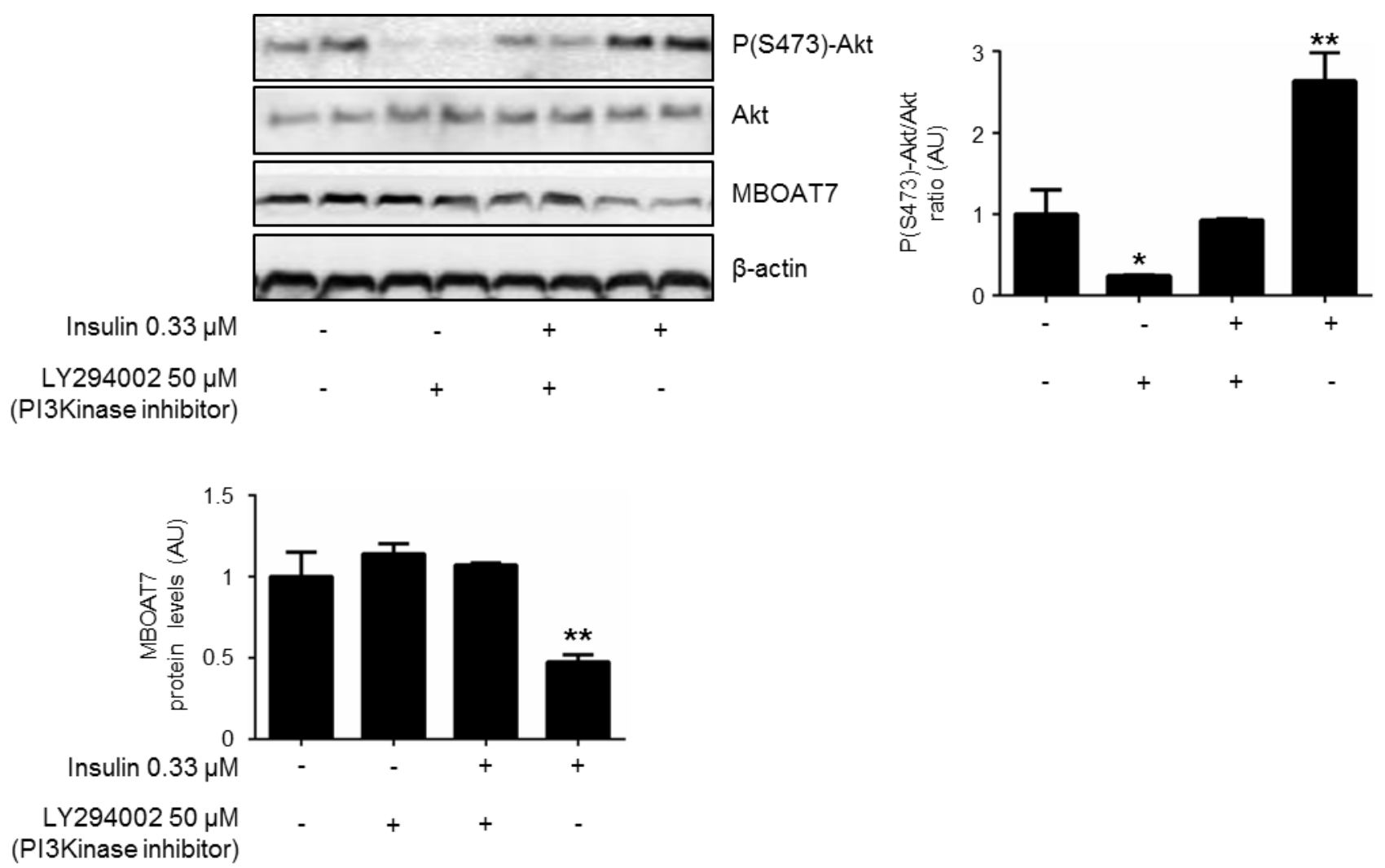

Fig. 14: Mboat7 down-regulation is dependent on insulin signaling activation in primary hepatocytes. Western blot and quantification of pThr308-Akt/Akt ratio and Mboat7 protein levels in hepatocytes isolated from wild- 
type mice and treated with insulin $(0.33 \mu \mathrm{M})$, LY294002 $(50 \mu \mathrm{M})$ or a combination of both for 6 hours; *p<0.05, $* * \mathrm{p}<0.01$ compared to wild-type untreated cells.

Conversely, mammalian target of Rapamycin (mTOR) pharmacological inhibition did not restore Mboat7 expression (Fig. 15).
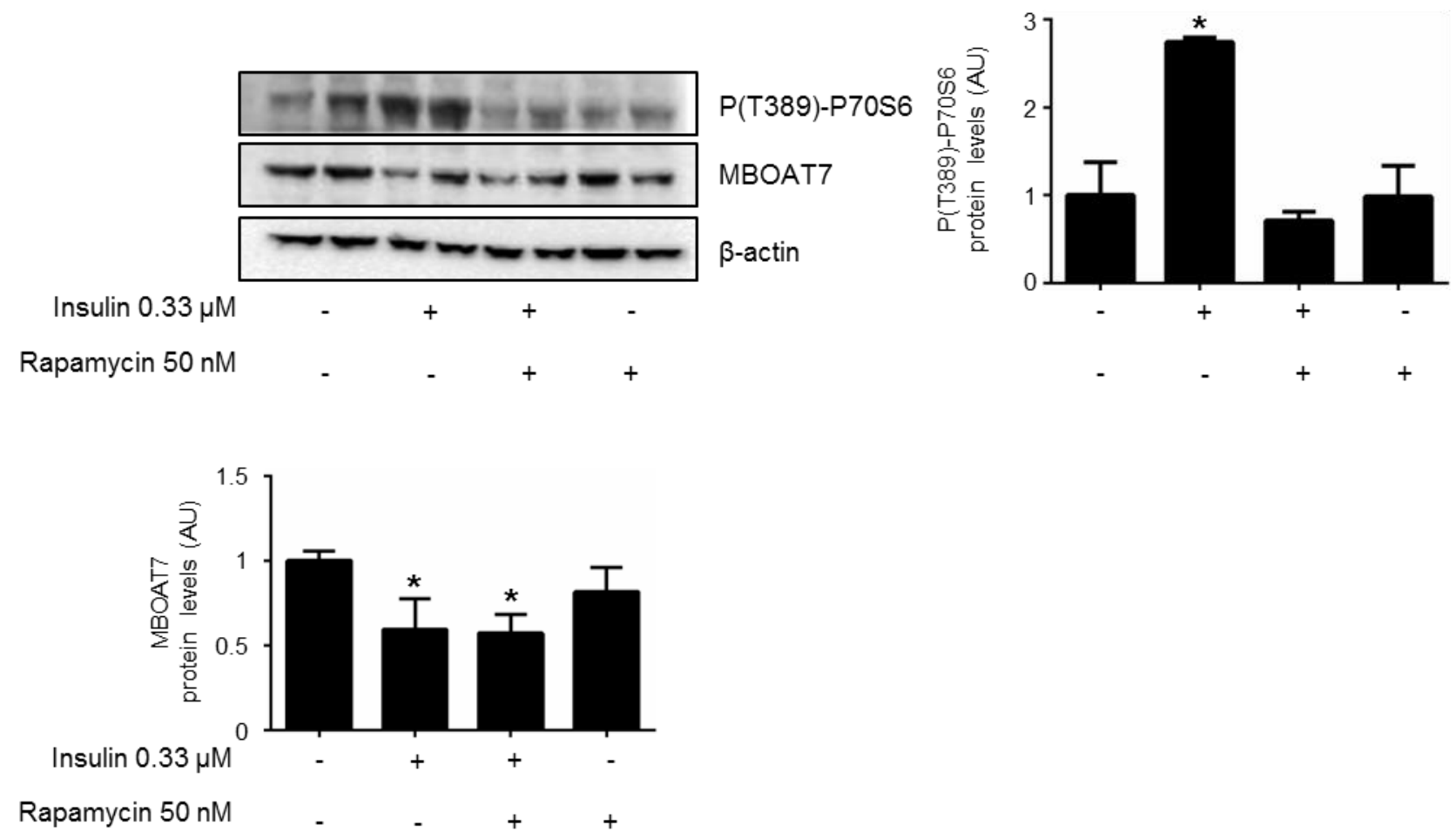

Fig. 15: mTORC1 inhibition does not affect the insulin-dependent MBOAT7 down-regulation. Western blot and quantification of pThr389-P70S6 kinase and Mboat7 protein levels in hepatocytes isolated from wild-type mice and treated with insulin $(0.33 \mu \mathrm{M})$, Rapamycin $(50 \mathrm{nM})$ or a combination of both for 6 hours; ${ }^{*} \mathrm{p}<0.05, * * \mathrm{p}<0.01$ compared to wild-type untreated cells.

These data suggest that insulin down-regulates Mboat7 protein expression in hepatocytes in a PI3Kinase-depedent manner. Consistently, in hepatocytes haplo-insufficient for the insulin receptor (InsR+/-) insulin-mediated suppression of Mboat7 was lost ( $\mathrm{p}<0.01$ vs. untreated hepatocytes; Fig. 16). 


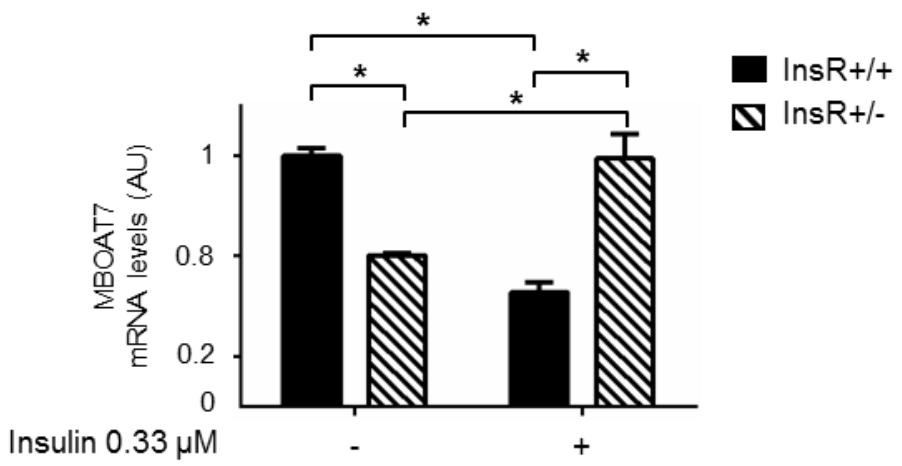

Fig. 16: In InsR+/- hepatocytes, the insulin-mediated suppression of Mboat7 expression is lost. Mboat7 expression in hepatocytes isolated from wild type and InsR+/- mice and treated with insulin $(0.33 \mu \mathrm{M})$ for 6 hours was evaluated by qRT-PCR, normalized for $\beta$-actin and expressed as fold increase (Arbitrary Units - AU), compared to wild-type untreated hepatocytes. ${ }^{*} \mathrm{p}<0.05, * * \mathrm{p}<0.01$.

\subsection{Mboat7 down-regulation by insulin in primary mouse hepatocytes requires FoxO1}

To investigate the mechanism of regulation of MBOAT7 expression by insulin signaling, we next focused on clarifying the specific transcription factors, involved in the insulin-dependent mechanisms of MBOAT7 regulation. On this purpose, we analyzed the sequence of MBOAT7 promoter, using the bioinformatics software Genomatix. This software predicted the presence of the Forkhead family transcription factor binding sites, among which Forkhead Box O1 (FoxO1) and Forkhead Box A2 (FoxA2) in MBOAT7 promoter, exactly localized close to the transcription start site of the MBOAT7 main isoform (isoform 1, $55 \mathrm{kDa}$ ). A scheme of MBOAT7 promoter is illustrated in Fig. 17. The presence of a binding site for FoxA2 on MBOAT7 promoter was previously confirmed by a chip-seq peak in hepatocytes, at the same position predicted by Genomatix (founded in the database Harmonizone, http://amp.pharm.mssm.edu/Harmonizome/). FoxO1 and FoxA2 are key mediators of insulin signaling at transcriptional level, regulating gluconeogenesis. Insulin signaling through Akt 
activation is a negative regulator of these two transcription factors, directly determining their phosphorylation and exclusion from the nucleus to cytoplasm and their consequent degradation. Thus, FoxO1 and FoxA2 may represent good candidates to mediate the insulin-dependent effect on MBOAT7 down-regulation.

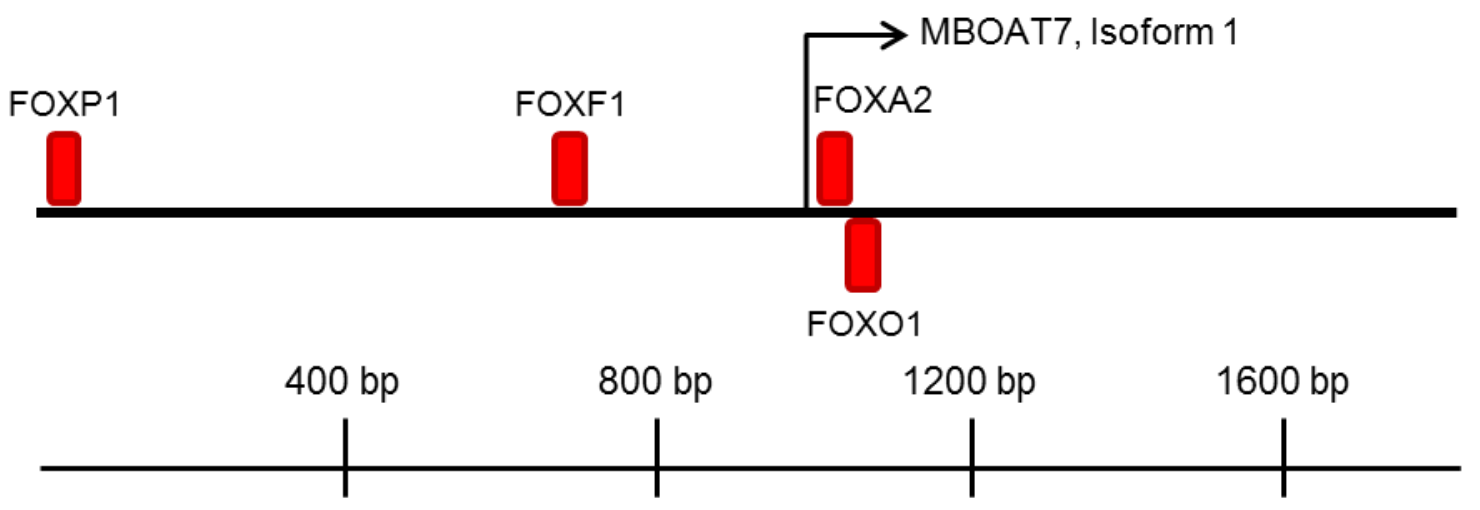

Matrix family: FKHD (Match total: 4)

Software: Genomatix \& Harmonizome

Fig. 17: FoxO1 and FoxA2 are predicted to bind to MBOAT7 promoter. The Fkhd family transcription factor binding sites and the localization of their consensus sequences on MBOAT7 promoter (isoform 1) were predicted by the bioinformatics software Genomatix. The presence of a binding site for FoxA2 in hepatocytes on MBOAT7 promoter was confirm by the chip-seq peak, revealed by Harmonizome. ${ }^{*} p<0.05$ for matrix family enrichment.

For this reason, we further characterize the effect of FoxO1 and/or FoxA2 deletion by exploiting morpholino antisense oligonucleotides (MPO) for silencing these transcription factors in wild-type primary mouse hepatocytes. FoxO1-MPO hepatocytes showed 50\% reduced FoxO1 protein expression ( $\mathrm{p}<0.05$ vs. untreated hepatocytes). Similar to what occurred in InsR+/- hepatocytes, the insulin-dependent Mboat7 down-regulation was abrogated in FoxO1-MPO hepatocytes ( $\mathrm{p}<0.05$ vs. 
FoxO1-MPO hepatocytes in absence of insulin stimulation), suggesting that FoxO1 is required to mediate the Mboat7 regulation by insulin (Fig. 18).
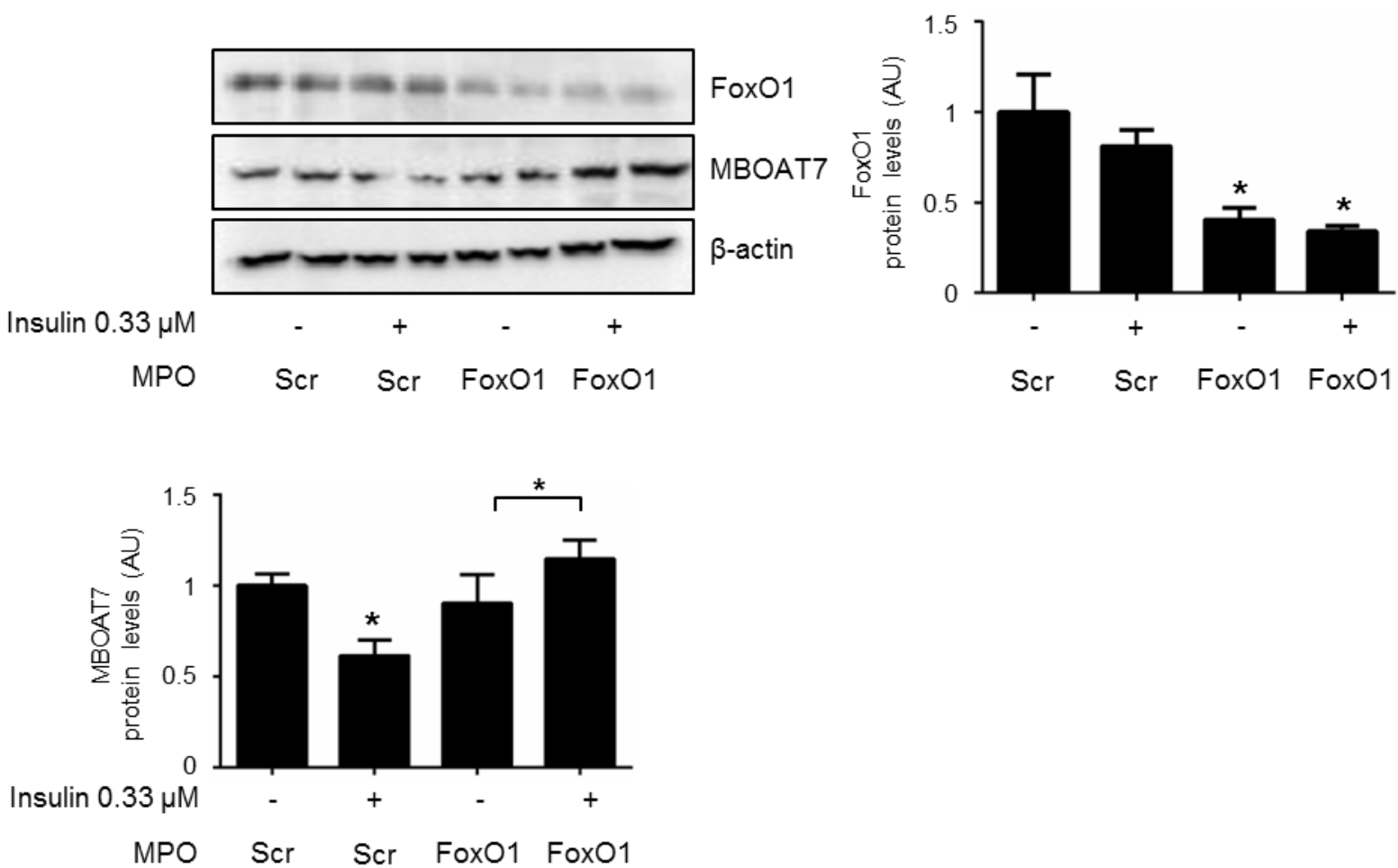

Fig. 18: Mboat7 down-regulation by insulin in primary mouse hepatocytes requires FoxO1. Hepatocytes isolated from wild type mice were treated with FoxO1-specific morpholino antisense oligonucleotides (MPO) or scramble $(\mathrm{Scr})(10 \mu \mathrm{M})$ administered for 24 hours, in presence or not of insulin $(0.33 \mu \mathrm{M})$; FoxO1 protein levels were normalized for $\beta$-actin. Mboat7 protein levels were evaluated in wild-type hepatocytes treated with FoxO1-MPO or scramble, in presence or not of insulin $(0.33 \mu \mathrm{M}) ;{ }^{*}<<0.05, * * \mathrm{p}<0.01$ compared to Scr.

Remarkably, the hampered Mboat7 expression that we had observed in InsR+/- hepatocytes compared to wild-type was avoided by FoxO1-MPO treatment $(\mathrm{p}<0.05$ vs. InsR+/- hepatocytes treated with scramble; Fig. 19A). The same result was obtained in mice fed MCD for 3 weeks, after i.v. weekly injection of FoxO1-MPO (p<0.05 vs. Scr-MPO mice fed MCD diet; Fig. 19B). 

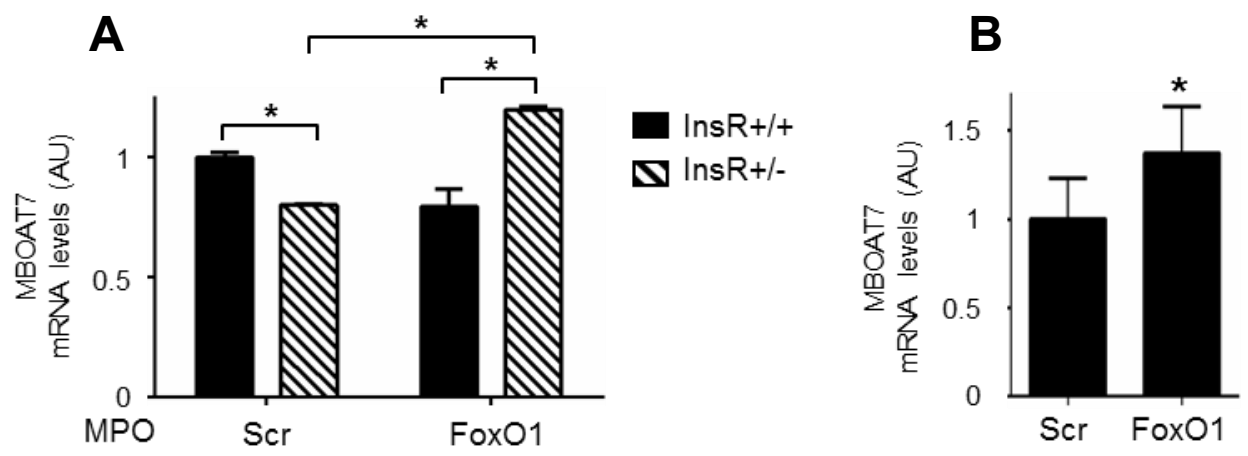

Fig. 19: InsR+/- treated with FoxO1-MPO re-stabilize Mboat7 mRNA levels comparable to that observed in wild-type. Mboat7 mRNA levels were evaluated in wild type $(\mathrm{InsR}+/+)$ and InsR $+/-$ hepatocytes treated with FoxO1-MPO or scramble; ${ }^{*} \mathrm{p}<0.05,{ }^{*} \mathrm{p}<0.01$ compared to InsR+/+ Scr $(\mathbf{A})$. InsR+/- mice were treated with 12.5 $\mathrm{mg} / \mathrm{kg}$ of FoxO1-MPO or scramble administered i.v. once weekly for 3 weeks during MCD feeding ( $\mathrm{n}=5$ mice per group); Mboat7 mRNA levels were normalized for $\beta$-actin; *p<0.05 compared to Scr (B).

\subsection{Hepatic Mboat7 silencing induces microvesicular steatosis in vivo}

Finally, to determine the impact of Mboat7 down-regulation on hepatic steatosis development, we silenced Mboat7 in wild-type male mice, by i.v. administration for 4 consecutive days of specific MPO. Biochemical features of wild-type mice treated with Scramble and MPO Mboat7 are shown in

\section{Table 8.}


Table 8. Biochemical features of wild-type mice, treated with Scramble and MPO Mboat7.

\begin{tabular}{lccc}
\hline & Scramble & MPO Mboat7 & P value \\
\hline Liver/Body Weight & $\mathbf{0 . 0 4} \pm \mathbf{0 . 0 0 3}$ & $\mathbf{0 . 0 4 5} \pm \mathbf{0 . 0 0 4}$ & $\mathbf{0 . 0 9}$ \\
Glucose (mg/dL) & $94 \pm 26$ & $84 \pm 32$ & 0.3 \\
Insulin (ng/mL) & $1.07 \pm 0.03$ & $1.02 \pm 0.07$ & 0.16 \\
Cholesterol (mg/dL) & $104 \pm 23.5$ & $129 \pm 24.3$ & 0.09 \\
Triglycerides (mg/dL) & $93 \pm 9.5$ & $108 \pm 31$ & 0.2 \\
FFAs (nmol/uL) & $0.69 \pm 0.23$ & $0.9 \pm 0.15$ & $\mathbf{0 . 0 7}$ \\
ALT (mg/dL) & $46[44-52]$ & $46[42-49]$ & 0.43 \\
\hline
\end{tabular}

Values are expressed as mean \pm standard deviation $(\mathrm{SD})$ or median [interquartile range]. $\mathrm{N}=6$ mice per group.

MPO induced a $45 \%$ silencing of hepatic Mboat7 ( $<<0.05$ vs. Scr-MPO; Fig. 20A), which comparable to that associated with carriage of the genetic risk variant in humans. This resulted in a $80 \%$ increase in hepatic triglyceride content $(\mathrm{p}<0.05$ vs. Scr-MPO; Fig. 20B) and in microvesicular steatosis development, but without altering circulating glucose, insulin and lipid levels (Table 8). These data are consistent with the notion that Mboat7 down-regulation has a causal role in the pathogenesis of steatosis, consistently with genetic epidemiological data $(60,61)$. 
A
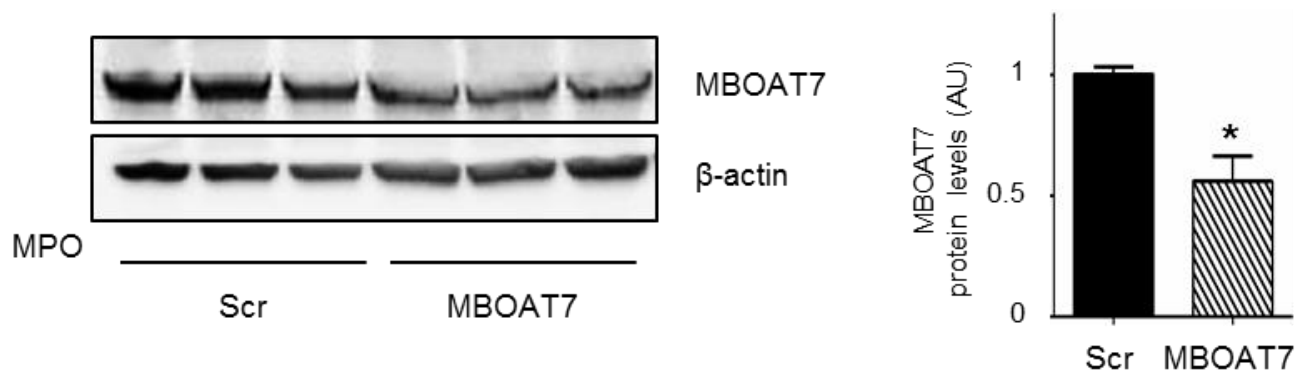

B
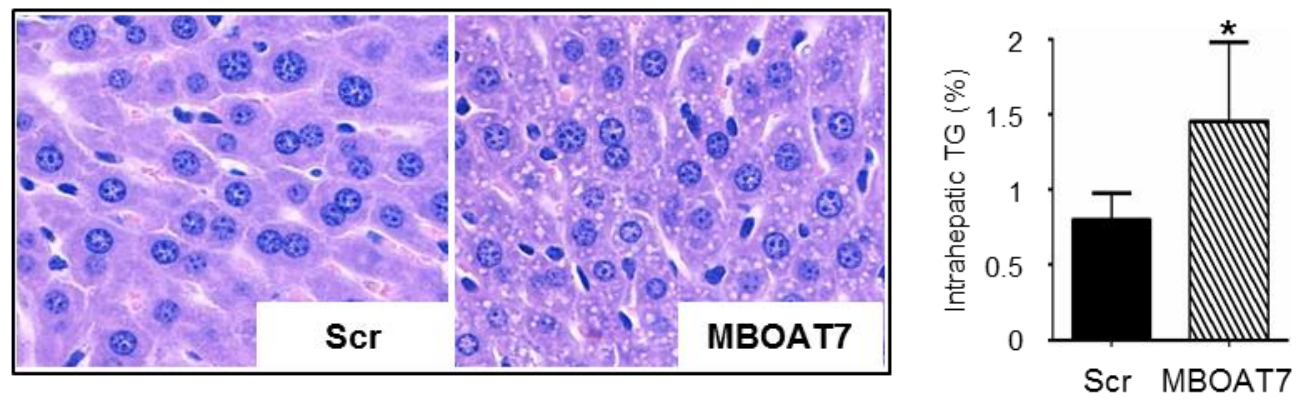

Fig. 20: Hepatic Mboat7 silencing induces microvesicular steatosis in vivo. Wild-type mice were treated with $12.5 \mathrm{mg} / \mathrm{kg}$ of Mboat7-MPO or scramble (Scr) administered i.v. daily for 4 days ( $\mathrm{n}=6$ per group) and were fasted for 16 hours before sacrifice. Western blot and quantification of hepatic Mboat7 protein levels were normalized for $\beta$-actin; * ${ }^{*}<0.05$ compared to Scr mice (A). H\&E staining and intrahepatic TG content assessment in Mboat7-MPO or Scr mice (B). Original magnification: 400X.

Steatosis development in mice silenced for Mboat7 was apparently not explained by induction of de novo lipogenesis. Conversely, hepatic Srepb1c and Fasn mRNA levels were markedly downregulated ( $<<0.01 v s$. Scr-MPO; Fig. 21A), possibly in an attempt to compensate for increased steatosis by suppressing lipogenesis. On the other hand, expression of genes regulating $\beta$-oxidation (Ppar $\alpha$ and Cpt1) and VLDL export (Mttp and ApoB100) were not affected by Mboat7 silencing (Fig. 21B, C). However, mRNA expression of fatty acids transporters (Fatp1 and Fabp1) was significantly upregulated in Mboat7-MPO mice vs. controls ( $\mathrm{p}<0.01$ and $\mathrm{p}<0.05$ vs. Scr-MPO respectively; Fig. 21D). 
Next, in order to validate these findings in the clinic, we then evaluated the mRNA expression of FATP1 in 119 obese patients of bariatric surgery cohort. At bivariate analysis, we found a positive correlation between FATP1 gene expression and the presence of T2DM (estimate $+0.76 \pm 0.28$; $\mathrm{p}=0.007$ ) and an inverse correlation with decreased MBOAT7 hepatic expression (estimate $-0.32 \pm 0.15$; $\mathrm{p}=0.03$ ). These results support the hypothesis that the predisposition to hepatic fat accumulation associated with down-regulation of hepatic MBOAT7 may be related to an increase in the uptake of FFAs from circulation into hepatocytes.
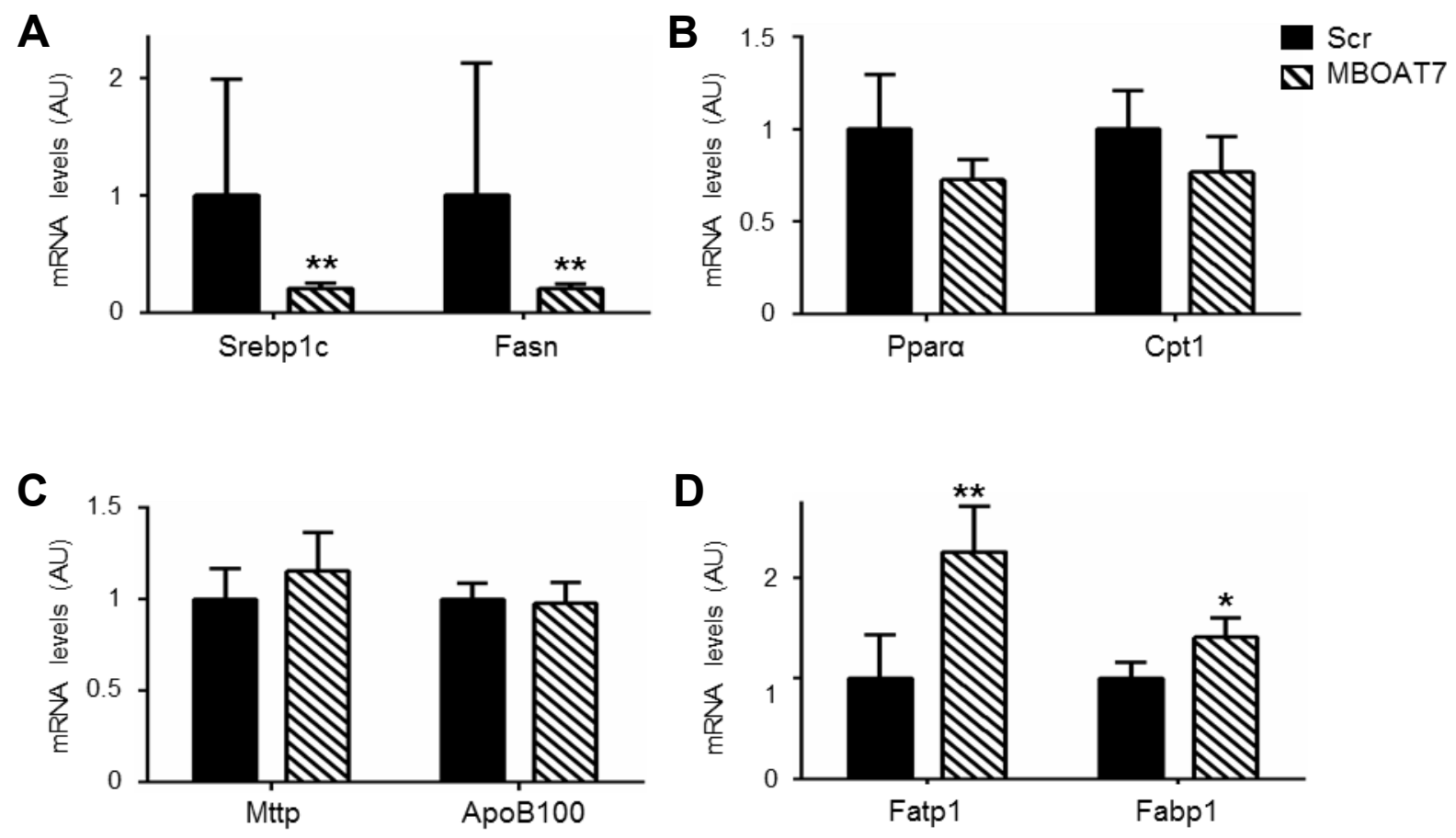

Fig. 21: Steatosis development in Mboat7-MPO mice is apparently explained by enhanced expression of fatty acids transporters. Hepatic Srebp1c and Fasn (genes involved in de novo lipogenesis) (A), Ppar $\alpha$ and Cpt1 (genes involved in $\beta$-oxidation) (B), Mttp and ApoB100 (lipid exporting marker) (C), Fatp1 and Fabp1 (fatty acids transporters) (D) expression was evaluated by qRT-PCR; mRNA levels were normalized to $\beta$-actin. Data are expressed as fold increase compared to Scr mice ( $\mathrm{n}=6$ mice per group; $\left.{ }^{*} \mathrm{p}<0.05,{ }^{*} \mathrm{p}<0.01\right)$. 
On the other hand, Mboat7 silencing was not associated with alterations in the hepatic expression of inflammatory cytokines and chemokines (such as Tnf- $\alpha$ and Cxcl10) (Fig. 22A), suggesting that, at least in this acute model, it exerts its main effect on liver disease by predisposing to hepatic fat accumulation, and not on inflammation. Furthermore, there was no modification in the expression of genes related to Mboat7 (Tmc4 at the linked locus and the paralogue Mboat5; Fig. 22B) was identified, highlighting the impossibility of Mboat5 to compensate to Mboat7 reduction.
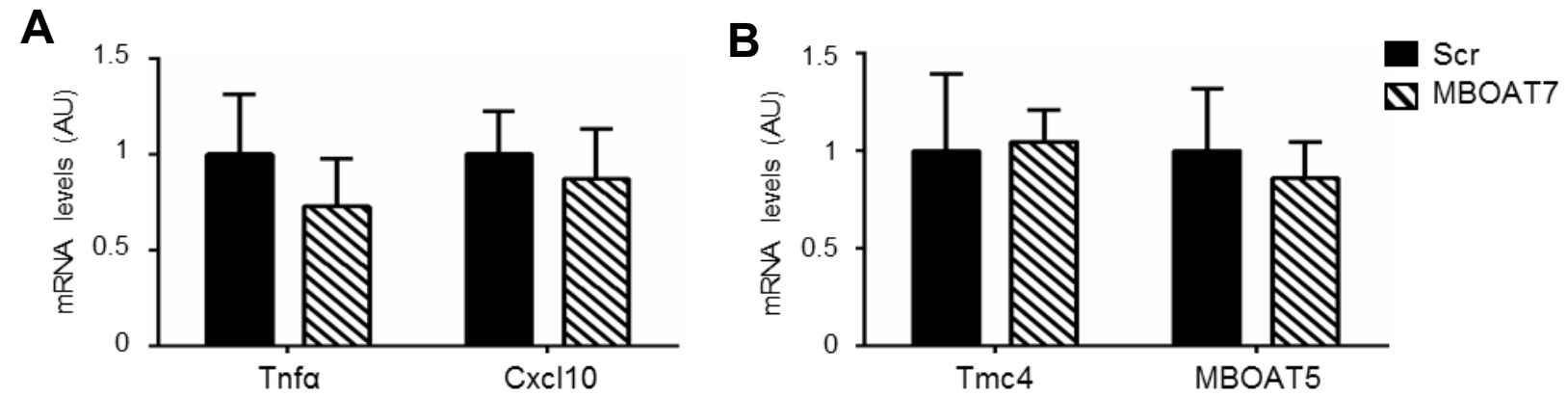

Fig. 22: No alteration in inflammation or in genes related to Mboat7 is identified in Mboat7-MPO mice. Tnfa and Cxcl10 (inflammatory markers) (A) and Tmc4 and Mboat5 (B) expression was evaluated by qRT-PCR; mRNA levels were normalized to $\beta$-actin. Data are expressed as fold increase compared to Scr mice ( $\mathrm{n}=6$ mice per group; $* \mathrm{p}<0.05, * * \mathrm{p}<0.01)$.

The microvesicular steatosis development and the enhanced expression of fatty acids transporters in Mboat7-silenced mice was associated with slightly increased circulating FFAs compared to Scr-MPO mice (Table 8), possibly due to Mboat7 silencing also in adipose tissue ( $\mathrm{p}<0.05$ vs. Scr-MPO; Fig. 23A), leading to increased FFAs flux from adipose tissue to the liver. Notably, MPO did not affect Mboat7 protein levels in brain, probably because it did not pass the hemato-encephalic barrier (Fig. 23B). 

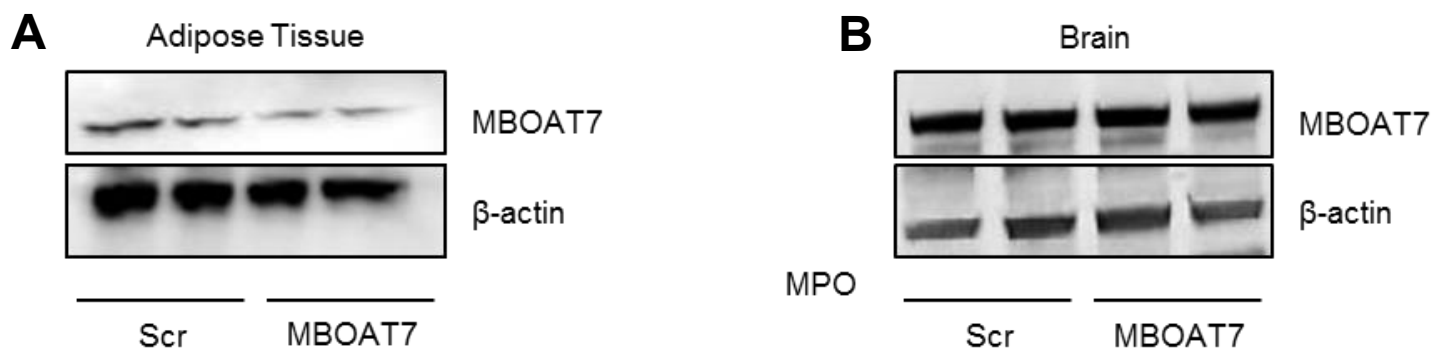

Fig. 23: Mboat7 is silenced also in adipose tissue of Mboat7-MPO mice, but not in brain. Western blot and quantification of Mboat7 protein levels in adipose tissues (A) and brains (B) from Mboat7-MPO or Scr mice fasted for 16 hours and normalized for $\beta$-actin; * $p<0.05$ compare $d$ to Scr mice.

For this reason, we assessed also serum FFAs in 72 lean NAFLD patients (Hepatology service cohort), stratified by the presence of MBOAT7 $\mathrm{T}$ allele of risk, in order to minimize the confounding effect of obesity. Demographic and clinical features of subjects included are shown in Table 2. At multivariate analysis, applying a generalized linear model, T2DM (estimate $+0.03 \pm 0.008 ; \mathrm{p}=0.0006)$ and MBOAT7 risk allele (estimate $+0.01 \pm 0.005 ; \mathrm{p}=0.02$ ) were independently associated with FFAs circulating concentration (Table 9) after adjustment for age, sex, BMI, presence of T2DM, PNPLA3, I148M alleles and MBOAT7, T alleles. These data are consistent with the hypothesis that in NAFLD patients the down-regulation of hepatic MBOAT7, caused by the presence of $\mathrm{T}$ allele may be involved in enhanced uptake of FFAs to the liver, promoting early stages of liver steatosis development. 
Table 9: Variables associated with FFAs dosage $(\mathrm{nmol} / \mu \mathrm{L})$ at multivariate generalized linear model analysis in 72 NAFLD patients (Hepatology cohort).

\begin{tabular}{lcc}
\hline & Estimate SE & P value \\
\hline Age, years & $0.0005 \pm 0.0003$ & 0.15 \\
Sex, F & $0.007 \pm 0.006$ & 0.25 \\
BMI, Kg/m ${ }^{2}$ & $-0.001 \pm 0.001$ & 0.21 \\
T2DM, yes & $0.03 \pm 0.008$ & $\mathbf{0 . 0 0 0 6}$ \\
PNPLA3, I148M alleles & $-0.006 \pm 0.006$ & 0.29 \\
MBOAT7, T alleles & $0.01 \pm 0.005$ & $\mathbf{0 . 0 2}$ \\
\hline
\end{tabular}

BMI: body mass index; T2DM: type 2 diabetes mellitus. 


\section{Discussion}

In this study we examined whether MBOAT7 down-regulation may be involved in mediating the effect of IR in the pathogenesis of liver disease by favoring steatosis development. To this end, we first showed that hepatic MBOAT7 expression is associated with the severity of inflammation and T2D in patients at risk of NASH, independently of the risk genotype. Association of hepatic Mboat7 downregulation with hepatic steatosis was confirmed in experimental models, but importantly IR induced down-regulation of Mboat7 independently of steatosis. Furthermore, we went on to show that Mboat7 is physiologically regulated by fasting-feeding cycles in the liver, depending on activation of insulin signaling in primary hepatocytes via a InsR/Akt/FoxO1 dependent pathway. Finally, acute downregulation of hepatic Mboat7 in vivo in mice determined the development of microvesicular steatosis by a mechanism encompassing up-regulation of fatty acids importers in hepatocytes, which was mirrored in patients carrying MBOAT7 T risk allele.

In the first part of this study, we evaluated whether the blunted expression of MBOAT7 may be involved in the development and progression of NAFLD, independently of the presence of MBOAT7 rs641738 C>T variant, which has been previously associated with steatosis onset and progressive NAFLD $(60,63)$. Moreover, we examined the clinical and genetic determinants of hepatic MBOAT7 expression in severely obese patients at risk of NASH. We found that hepatic MBOAT7 expression progressively decreased with liver damage severity. In particular, impaired fasting glucose/type 2 diabetes mellitus (IFG/T2DM), necroinflammation and the presence of the $\mathrm{T}$ risk allele were independently associated with reduced MBOAT7 expression. Nonetheless, in these patients IFG/T2DM and MBOAT7 risk allele were independently associated with MBOAT7 hampered expression also in adipose tissue. Given the complex interplay between liver and adipose tissue in IR development, that is the strongest predictor of progressive NAFLD, MBOAT7 down-regulation in these tissues in IR 
conditions may further contribute to liver damage development and progression independently of MBOAT7 genotype. To note, the MBOAT7 variant has been recognized as a risk factor for liver damage severity also in different aetiologies $(64,65)$, suggesting that the MBOAT7 dumped expression may represent a common mechanism of liver injury.

MBOAT7 is involved in the reacylation of phospholipids as part of the remodeling pathway 'Land's cycle' by specifically transferring polyunsaturated acyl-CoAs, such as arachidonoyl-CoA, to lysophosphatidylinositol and other lysophospholipids, thereby allowing to achieve an adequate level of desaturation of the membranes and to maintain a low amount of free arachidonic acid, that is a potent trigger for liver inflammation and fibrosis. Carriers of the rs641738 $\mathrm{T}$ risk allele had reduced expression and synthesis of the MBOAT7 protein in the liver and changes in plasma phosphatidylinositol species consistent with decreased MBOAT7 activity $(60,61)$. These observations suggest that reduced enzymatic activity of MBOAT7 may explain, on one side, the increased liver fat accumulation and the steatosis onset, and on the other side, liver damage worsening into NASH and advanced fibrosis. Indeed, higher levels of circulating bioactive proinflammatory metabolites of arachidonic acid have been reported in patients with NASH. Evaluation of MBOAT7 expression in primary cultures of specific human cell populations are consistent with this hypothesis. Specifically, the rs641738 T risk allele was associated with reduced MBOAT7 expression in hepatocytes, but not in hHSCs, thereby suggesting that hepatocellular MBOAT7 down-regulation may directly account for predisposition to TG accumulation by altering the biochemical properties of cell membranes. However, in lymphocytes and monocytes, characterized by high MBOAT7 expression, the T risk allele was also associated with down-regulation of gene expression, which was also observed during cell activation. We could therefore hypothesize that MBOAT7 down-regulation may be involved in priming macrophages for triggering inflammation, thus determining the switch from simple steatosis to steatohepatitis. 
In line with this hypothesis, in the second part of this study, we showed that MBOAT7 expression was reduced in experimental models of NAFLD, such as MCD diet, but more so in genetically obese Lep ${ }^{o b / o b}$ mice which develop IR and in Insulin receptor haplo-insufficient mice (InsR $+/-)$, characterized by hyperinsulinemia despite the lack of increased adiposity and of significant steatosis. A possible explanation could be due to the fact that despite the significantly reduced gene expression, Mboat7 protein levels only slightly decreased in InsR+/- mice fed standard diet compared to wild-type, preserving its total enzymatic activity more than in patients carriers $\mathrm{T}$ allele of risk or in acute silencing, where this reduction is more pronounced. Thus, we detected a strong link between Mboat7 down-regulation and hyperinsulinemia associated with IR, but not with steatosis alone.

We hypothesized that insulin signaling may be responsible for down-regulation of MBOAT7 expression during NAFLD, thereby linking IR to the development of fatty liver and steatohepatitis. To test this, we next examined the physiological regulation of hepatic Mboat7 during the transition from fasting to refeeding in vivo in mice. Indeed, we observed that hepatic Mboat7 expression decreased during refeeding in insulin responsive tissues (liver, adipose tissue and proximal intestine), concomitantly with the rise of insulin levels and the activation of hepatic insulin signaling through PI3K and AKT.

Then, to gain insight into the physiological impact of Mboat7 regulation by insulin, we compared the expression pattern to that of Mboat5, the closest paralogue which has been shown to favor lipoproteins secretion and whose hepatic specific silencing has been demonstrate to induce steatosis $(82,83)$. We observed that Mboat5 was similarly down-regulated following refeeding in the liver. However, during hyperinsulinemia Mboat7 and Mboat5, showed a divergent regulation, with upregulation of Mboat5 expression. This may result in amelioration of saturated free fatty acid-induced ER stress, lowering also blood glucose and insulin levels in Lep ${ }^{o b / o b}$ mice $(81,84)$. Furthermore, Mboat5 is regulated by liver $\mathrm{X}$ receptors (LXR), that is generally active in experimental models of IR 
(81, 84). Conversely, mice with specific deletion of hepatic Mboat5 display decreased arachidonic acid-containing phospholipids in the liver and an increased risk of hepatic steatosis onset, due to altered lipid kinetics within hepatocytes, and inflammation $(82,83)$.

The next question we addressed is whether and how insulin regulates hepatic Mboat7 expression. To test whether insulin has a direct causal role in the regulation of Mboat7 expression, we isolated primary mouse hepatocytes and demonstrated that exposure to insulin was able to downregulate Mboat7, suggesting this is a cell autonomous property of hepatocytes. In keeping with these results, we showed, by pharmacological inhibition, that insulin down-regulation of Mboat7 is dependent on the activation of the PI3Kinase.

The transcription factors FoxO1 and FoxA2 may represent good candidates to mediate the insulin-dependent effect on Mboat7 down-regulation. FoxO1 and FoxA2 are key mediators of insulin signaling at transcriptional level, regulating gluconeogenesis and ketogenesis. Akt activation is a negative regulator of FoxO1 and FoxA2, determining their phosphorylation, transcriptional inactivation and exclusion from the nucleus to cytoplasm and their consequent degradation $(19,90)$. Interestingly, we predicted by bioinformatics algorithms the presence of binding sites for these transcription factor, in MBOAT7 promoter, close to the transcription start site of the MBOAT7 main isoform. Supporting a role of FoxO1 in mediating insulin-dependent regulation of Mboat7, FoxO1 deletion in wild-type primary mouse hepatocytes is similar to that occurred in InsR+/- hepatocytes, it impairs the insulindependent Mboat7 down-regulation, suggesting that FoxO1 is one of the transcription factor required to mediate the Mboat7 regulation by insulin. Consistently, the down-regulation of Mboat7 expression observed in InsR+/- genotype was rescued by FoxO1 silencing. These data are consistent with the possibility that FoxO1 may regulate Mboat7, although they do not formally prove that this gene is a direct target of this transcription factor. Indeed, FoxO1 is able to function both as a coactivator or as a corepressor of the transcription of target genes. Specifically, FoxO1 haplo-insufficiency normalized the 
expression of glucose-6-phosphatase (G6pc) and phosphoenolpyruvate carboxykinase (Pck1), ratelimiting enzymes for glycogenolysis and gluconeogenesis, respectively, in insulin resistant mice (i.e. InsR+/-), in which FoxO1 activation is predominant $(91,92)$. In fact, in IR conditions, insulin fails to inactivate FoxO1 further inducing hepatic glucose output (23). However, the expression of G6pc and Pck1 in InsR+/-FoxO1+/- is comparable to wild-type mice, suggesting that their expression may be supported by other FoxO isoforms, such as FoxO3, that is closely related to FoxO1 and it possesses the ability to regulate transcription of reporter genes containing insulin response elements from the G6p and Pck1 promoters in an insulin-dependent manner (91). As we speculate that FoxO1 is not the only mediator of Mboat7 down-regulation by insulin (as FoxO1 silencing did not completely shut down Mboat7), we plan to study the also the impact of FoxA2 silencing. Interestingly, FoxA2 binding to the Mboat7 promoter was already directly confirmed by a chip-seq peak in hepatocytes, at the same position predicted by the bioinformatics software Genomatix. In particular, the hepatic regulation of FoxA2 phosphorylation by insulin-stimulated IRS signaling is not impaired in hyperinsulinemia or IR states, where FoxA2 is found exclusively in the cytoplasm of hepatocytes, despite the defect in hepatic insulin signaling $(17,24)$. The different regulation of FoxO1 and FoxA2 in IR may be explained by the preferential phosphorylation of FoxO1 through insulin-IRS2 signaling, which is impaired in hepatic IR, whereas FoxA2 can be phosphorylated by either insulin-IRS1 or insulin-IRS2 signaling (17). Moreover, FoxA2 is directly regulated by glucagon, by determining its acetylation at the conserved residue Lys259 and promoting its transcriptional activity. Therefore, FoxA2 is transcriptionally active in the fasted state (16-18), in which Mboat7 is more expressed.

Finally, in the third part of this study we sought to confirm in in vivo models whether Mboat7 down-regulation impact on development of fatty liver in vivo. We showed that acute Mboat7 silencing, achieving about $50 \%$ down-regulation of protein levels by i.v. injections of MPO antisense oligonucleotides for 4 consecutive days resulted in $80 \%$ increase in hepatic TG content and in 
microvesicular steatosis development, without altering circulating glucose, insulin and lipid levels. Steatosis development was apparently not explained by altered expression of genes regulating de novo lipogenesis or $\beta$-oxidation, but was associated with induction of the main hepatocellular fatty acids transporters (Fatp1, Fabp1). Supporting the hypothesis that the predisposition to hepatic fat accumulation due to Mboat7 down-regulation may be related to an increased FFAs import from the circulation into hepatocytes, we found that in the bariatric surgery cohort hepatic FATP1 was inversely correlated with MBOAT7 expression. Interestingly, microvesicular steatosis development in Mboat7silenced mice was associated with slightly increased circulating FFAs, possibly due to Mboat7 silencing also in adipose tissue, leading to increased FFAs flux from adipose tissue to the liver. However, previous studies did not detect any impact of the MBOAT7 risk alleles on the total amount of circulating free fatty acids in humans $(60,61)$.

Differently from this previous data, to minimize the confounding effect of obesity we assessed also serum FFAs in 72 lean NAFLD patients (with a mean value of BMI of 26), stratified by the presence of MBOAT7 $\mathrm{T}$ risk allele. In this cohort of patients T2DM and MBOAT7 risk allele were independently associated with FFAs circulating concentration, consistently with the hypothesis that in NAFLD patients the down-regulation of adipose tissue MBOAT7, caused by the presence of $\mathrm{T}$ allele may be involved in triggering the phenotype by increasing FFAs availability, then facilitating the enhanced uptake of FFAs to the liver, promoting early stages of liver steatosis development. Of note, a significant interaction between MBOAT7 genotype and the presence of over-weight has been already recorded, in line with the hypothesis that the phenotypic expression of the mutation is triggered by the increased flux of fatty acids to the liver (93). We did not highlight differences across genotypes of MBOAT7 variant in circulating levels of FFAs in the large cohort of the DHS which includes more than 2000 individuals (60), possibly because these subjects have a mean value of BMI of 30, that is already considered suggestive of obesity and they have a different ethnicity. 
Finally, Mboat7 silencing was not directly associated with alterations in the hepatic expression of inflammatory cytokines and chemokines (such as Tnf- $\alpha$ and Cxcl10). A possible limitation of this study could be the lack of Eicosanoids measurement in sera from Mboat7-silenced mice. Indeed, the MBOAT7 reduced activity may influence more promptly the fluctuation of these pro-inflammatory mediators (61).

Another weakness of this study is the absence of Mboat7 KO mice model. In fact, Mboat7 plays a crucial role in brain development in mice, since arachidonic acid is the most enriched PUFA in the brain and it is involved in multiple aspects of neuronal development and function. Therefore, Mboat7 KO mice die within a month and show atrophy of the cerebral cortex and hippocampus, disordered cortical lamination and neuronal processes and delayed neuronal migration in the cortex (67, 73). A possible alternative in order to reproduce Mboat7 reduction may be a liver-specific Mboat7 silenced mice model, that we didn't assessed. This model could be useful to study deeply the effect of the reduced activity of Mboat7 on steatosis pathogenesis, further evaluating VLDL exporting processes from liver to peripheral tissues. Also a model of full $\mathrm{KO}$ in hepatocytes could be intriguing to further elucidate that the down-regulation of MBOAT7 is sufficient to predispose to steatosis in hepatocytes. However, these models are not representative of the complex cross-talk between the different insulinsensitive tissues in presence of Mboat7 reduction. Moreover, we didn't tested also the in vivo effect of acute insulin treatment on several insulin-responsible tissues, confirming the data obtained from endogenous production of insulin, due to the refeeding with chow. 


\section{Conclusions}

In summary, we found that hyperinsulinemia, a typical feature of metabolic syndrome and postprandial conditions, reduces hepatic MBOAT7 expression via increased insulin signaling activation through the Insulin receptor - PI3K - FoxO1 pathway. Reduced MBOAT7 then favors in turn hepatic fat accumulation. These data suggest that MBOAT7 down-regulation may be implicated in mediating the effect of hyperinsulinemia characteristics of the metabolic syndrome in determining the development of NAFLD and progressive liver disease in general.

We speculate that during fasting conditions MBOAT7 is localize specifically in the membranes, where it is able to catalyze the desaturation of phospholipids, maintaining their normal fluidity and the dynamism, that allows the exchange of the metabolites from the membranes to intracellular compartments, such as the mitochondria, thus favoring the $\beta$-oxidation processes. Conversely, in pathologic state or in post prandial hyperinsulinemia or in patients who carry the $\mathrm{T}$ risk allele, MBOAT7 is reduced in the membrane fractions, determining the saturation and the immobilization of phospholipids, creating a microenvironment that favors TG accumulation and steatosis onset, and promoting, by still not characterized mechanisms, the expression of hepatic lipid transporters. Moreover, the increased availability of free arachidonic acid may trigger liver inflammation and fibrosis, switching simple steatosis to steatohepatitis.

Future studies will be focused on the evaluation of the insulin-dependent mechanisms of MBOAT7 regulation, investigating the role of the transcription factors FoxO1 and/or FoxA2 by silencing their expression a) in vivo in experimental models of NAFLD (mice fed MCD and in Lep ${ }^{o b / o b}$ mice) and b) ex vivo in primary mouse hepatocytes by antisense oligonucleotides. We will plan also to investigate whether the effect of MBOAT7 down-regulation on TG accumulation is a cell-autonomous property of hepatocytes, by generating a MBOAT7 knock-out cell lines by using the Clustered 
Regularly Interspaced Short Palindromic Repeats (CRISPR)/CAS9 technology (94). On this purpose, we will sough to produce a MBOAT7 deletion in HepG2 cell lines and treat them with low concentration of saturated fatty acids, mimicking physiological condition and high concentrations, representative of pathological condition, in order to understand the exact mechanisms through which MBOAT7 down-regulation induces steatosis development, and the effect of MBOAT7 downregulation on specific fatty acids transporters. 


\section{References}

1. Browning JD, Szczepaniak LS, Dobbins R, Nuremberg P, Horton JD, Cohen JC, et al. Prevalence of hepatic steatosis in an urban population in the United States: impact of ethnicity. Hepatology (Baltimore, Md). 2004;40(6):1387-95.

2. Marchesini G, Brizi M, Morselli-Labate AM, Bianchi G, Bugianesi E, McCullough AJ, et al. Association of nonalcoholic fatty liver disease with insulin resistance. The American journal of medicine. 1999;107(5):450-5.

3. Marchesini G, Brizi M, Bianchi G, Tomassetti S, Bugianesi E, Lenzi M, et al. Nonalcoholic fatty liver disease: a feature of the metabolic syndrome. Diabetes. 2001;50(8):1844-50.

4. Angulo P, Kleiner DE, Dam-Larsen S, Adams LA, Bjornsson ES, Charatcharoenwitthaya P, et al. Liver Fibrosis, but No Other Histologic Features, Is Associated With Long-term Outcomes of Patients With Nonalcoholic Fatty Liver Disease. Gastroenterology. 2015;149(2):389-97.e10.

5. Marra F, Gastaldelli A, Svegliati Baroni G, Tell G, Tiribelli C. Molecular basis and mechanisms of progression of non-alcoholic steatohepatitis. Trends Mol Med. 2008;14(2):72-81.

6. Silverman JF, O'Brien KF, Long S, Leggett N, Khazanie PG, Pories WJ, et al. Liver pathology in morbidly obese patients with and without diabetes. The American journal of gastroenterology. 1990;85(10):1349-55.

7. Dongiovanni P, Valenti L. A Nutrigenomic Approach to Non-Alcoholic Fatty Liver Disease. International journal of molecular sciences. 2017;18(7).

8. Cohen JC, Horton JD, Hobbs HH. Human fatty liver disease: old questions and new insights. Science (New York, NY). 2011;332(6037):1519-23. 
9. Estep JM, Baranova A, Hossain N, Elariny H, Ankrah K, Afendy A, et al. Expression of cytokine signaling genes in morbidly obese patients with non-alcoholic steatohepatitis and hepatic fibrosis. Obesity surgery. 2009;19(5):617-24.

10. Malhi H, Gores GJ. Molecular mechanisms of lipotoxicity in nonalcoholic fatty liver disease. Semin Liver Dis. 2008;28(4):360-9.

11. Miele L, Grieco A, Armuzzi A, Candelli M, Forgione A, Gasbarrini A, et al. Hepatic mitochondrial beta-oxidation in patients with nonalcoholic steatohepatitis assessed by $13 \mathrm{C}$-octanoate breath test. The American journal of gastroenterology. 2003;98(10):2335-6.

12. Charlton M, Sreekumar R, Rasmussen D, Lindor K, Nair KS. Apolipoprotein synthesis in nonalcoholic steatohepatitis. Hepatology (Baltimore, Md). 2002;35(4):898-904.

13. Kahn SE, Hull RL, Utzschneider KM. Mechanisms linking obesity to insulin resistance and type 2 diabetes. Nature. 2006;444(7121):840-6.

14. Saltiel AR, Kahn CR. Insulin signalling and the regulation of glucose and lipid metabolism. Nature. 2001;414(6865):799-806.

15. Lin HV, Accili D. Hormonal regulation of hepatic glucose production in health and disease. Cell metabolism. 2011;14(1):9-19.

16. von Meyenn F, Porstmann T, Gasser E, Selevsek N, Schmidt A, Aebersold R, et al. Glucagoninduced acetylation of Foxa2 regulates hepatic lipid metabolism. Cell metabolism. 2013;17(3):436-47.

17. Wolfrum C, Asilmaz E, Luca E, Friedman JM, Stoffel M. Foxa2 regulates lipid metabolism and ketogenesis in the liver during fasting and in diabetes. Nature. 2004;432(7020):1027-32.

18. Wolfrum C, Besser D, Luca E, Stoffel M. Insulin regulates the activity of forkhead transcription factor Hnf-3beta/Foxa-2 by Akt-mediated phosphorylation and nuclear/cytosolic localization. Proceedings of the National Academy of Sciences of the United States of America. 2003;100(20):11624-9. 
19. Howell JJ, Stoffel M. Nuclear export-independent inhibition of Foxa2 by insulin. The Journal of biological chemistry. 2009;284(37):24816-24.

20. Dongiovanni P, Rametta R, Meroni M, Valenti L. The role of insulin resistance in nonalcoholic steatohepatitis and liver disease development--a potential therapeutic target? Expert review of gastroenterology \& hepatology. 2016;10(2):229-42.

21. Cefalu WT. Insulin resistance: cellular and clinical concepts. Experimental biology and medicine. 2001;226(1):13-26.

22. Grancini V, Trombetta M, Lunati ME, Zimbalatti D, Boselli ML, Gatti S, et al. Contribution of beta-cell dysfunction and insulin resistance to cirrhosis-associated diabetes: Role of severity of liver disease. Journal of hepatology. 2015;63(6):1484-90.

23. Matsumoto M, Pocai A, Rossetti L, Depinho RA, Accili D. Impaired regulation of hepatic glucose production in mice lacking the forkhead transcription factor Foxo1 in liver. Cell metabolism. 2007;6(3):208-16.

24. Silva JP, von Meyenn F, Howell J, Thorens B, Wolfrum C, Stoffel M. Regulation of adaptive behaviour during fasting by hypothalamic Foxa2. Nature. 2009;462(7273):646-50.

25. Yamaguchi K, Yang L, McCall S, Huang J, Yu XX, Pandey SK, et al. Inhibiting triglyceride synthesis improves hepatic steatosis but exacerbates liver damage and fibrosis in obese mice with nonalcoholic steatohepatitis. Hepatology (Baltimore, Md). 2007;45(6):1366-74.

26. Bugianesi E, Gastaldelli A, Vanni E, Gambino R, Cassader M, Baldi S, et al. Insulin resistance in non-diabetic patients with non-alcoholic fatty liver disease: sites and mechanisms. Diabetologia. 2005;48(4):634-42.

27. Fabbrini E, Mohammed BS, Magkos F, Korenblat KM, Patterson BW, Klein S. Alterations in adipose tissue and hepatic lipid kinetics in obese men and women with nonalcoholic fatty liver disease. Gastroenterology. 2008;134(2):424-31. 
28. Korenblat KM, Fabbrini E, Mohammed BS, Klein S. Liver, muscle, and adipose tissue insulin action is directly related to intrahepatic triglyceride content in obese subjects. Gastroenterology. 2008;134(5):1369-75.

29. Targher G, Day CP, Bonora E. Risk of cardiovascular disease in patients with nonalcoholic fatty liver disease. The New England journal of medicine. 2010;363(14):1341-50.

30. Ozcan U, Cao Q, Yilmaz E, Lee AH, Iwakoshi NN, Ozdelen E, et al. Endoplasmic reticulum stress links obesity, insulin action, and type 2 diabetes. Science (New York, NY). 2004;306(5695):45761.

31. Valenti L, Fracanzani AL, Fargion S. The immunopathogenesis of alcoholic and nonalcoholic steatohepatitis: two triggers for one disease? Seminars in immunopathology. 2009;31(3):359-69.

32. Miele L, Valenza V, La Torre G, Montalto M, Cammarota G, Ricci R, et al. Increased intestinal permeability and tight junction alterations in nonalcoholic fatty liver disease. Hepatology (Baltimore, Md). 2009;49(6):1877-87.

33. Marra F, Bertolani C. Adipokines in liver diseases. Hepatology (Baltimore, Md). 2009;50(3):957-69.

34. Bilzer M, Roggel F, Gerbes AL. Role of Kupffer cells in host defense and liver disease. Liver international : official journal of the International Association for the Study of the Liver. 2006;26(10):1175-86.

35. Bugianesi E, Leone N, Vanni E, Marchesini G, Brunello F, Carucci P, et al. Expanding the natural history of nonalcoholic steatohepatitis: from cryptogenic cirrhosis to hepatocellular carcinoma. Gastroenterology. 2002;123(1):134-40.

36. Dongiovanni P, Meroni M, Baselli GA, Bassani GA, Rametta R, Pietrelli A, et al. Insulin resistance promotes Lysyl Oxidase Like 2 induction and fibrosis accumulation in non-alcoholic fatty liver disease. Clinical science (London, England : 1979). 2017;131(12):1301-15. 
37. Dongiovanni P, Valenti L, Rametta R, Daly AK, Nobili V, Mozzi E, et al. Genetic variants regulating insulin receptor signalling are associated with the severity of liver damage in patients with non-alcoholic fatty liver disease. Gut. 2010;59(2):267-73.

38. Valenti L, Bugianesi E, Pajvani U, Targher G. Nonalcoholic fatty liver disease: cause or consequence of type 2 diabetes? Liver international : official journal of the International Association for the Study of the Liver. 2016;36(11):1563-79.

39. Kisseleva T, Cong M, Paik Y, Scholten D, Jiang C, Benner C, et al. Myofibroblasts revert to an inactive phenotype during regression of liver fibrosis. Proceedings of the National Academy of Sciences of the United States of America. 2012;109(24):9448-53.

40. Yoshimoto S, Loo TM, Atarashi K, Kanda H, Sato S, Oyadomari S, et al. Obesity-induced gut microbial metabolite promotes liver cancer through senescence secretome. Nature. 2013;499(7456):97101.

41. Coppe JP, Patil CK, Rodier F, Sun Y, Munoz DP, Goldstein J, et al. Senescence-associated secretory phenotypes reveal cell-nonautonomous functions of oncogenic RAS and the p53 tumor suppressor. PLoS biology. 2008;6(12):2853-68.

42. Dongiovanni P, Anstee QM, Valenti L. Genetic predisposition in NAFLD and NASH: impact on severity of liver disease and response to treatment. Current pharmaceutical design. 2013;19(29):5219-38.

43. Dongiovanni P, Romeo S, Valenti L. Genetic Factors in the Pathogenesis of Nonalcoholic Fatty Liver and Steatohepatitis. BioMed research international. 2015;2015:460190.

44. Dongiovanni P, Valenti L. Genetics of nonalcoholic fatty liver disease. Metabolism: clinical and experimental. 2016;65(8):1026-37. 
45. Romeo S, Kozlitina J, Xing C, Pertsemlidis A, Cox D, Pennacchio LA, et al. Genetic variation in PNPLA3 confers susceptibility to nonalcoholic fatty liver disease. Nature genetics. 2008;40(12):1461-5.

46. Pingitore P, Dongiovanni P, Motta BM, Meroni M, Lepore SM, Mancina RM, et al. PNPLA3 overexpression results in reduction of proteins predisposing to fibrosis. Human molecular genetics. 2016;25(23):5212-22.

47. Mondul A, Mancina RM, Merlo A, Dongiovanni P, Rametta R, Montalcini T, et al. PNPLA3 I148M Variant Influences Circulating Retinol in Adults with Nonalcoholic Fatty Liver Disease or Obesity. The Journal of nutrition. 2015;145(8):1687-91.

48. Dongiovanni P, Romeo S, Valenti L. Hepatocellular carcinoma in nonalcoholic fatty liver: role of environmental and genetic factors. World journal of gastroenterology. 2014;20(36):12945-55.

49. Donati B, Motta BM, Pingitore P, Meroni M, Pietrelli A, Alisi A, et al. The rs2294918 E434K variant modulates patatin-like phospholipase domain-containing 3 expression and liver damage. Hepatology (Baltimore, Md). 2016;63(3):787-98.

50. BasuRay S, Smagris E, Cohen JC, Hobbs HH. The PNPLA3 variant associated with fatty liver disease (I148M) accumulates on lipid droplets by evading ubiquitylation. Hepatology (Baltimore, Md). 2017.

51. Dongiovanni P, Donati B, Fares R, Lombardi R, Mancina RM, Romeo S, et al. PNPLA3 I148M polymorphism and progressive liver disease. World journal of gastroenterology. 2013;19(41):6969-78.

52. Valenti L, Motta BM, Soardo G, Iavarone M, Donati B, Sangiovanni A, et al. PNPLA3 I148M polymorphism, clinical presentation, and survival in patients with hepatocellular carcinoma. PloS one. 2013;8(10):e75982. 
53. Pirazzi C, Valenti L, Motta BM, Pingitore P, Hedfalk K, Mancina RM, et al. PNPLA3 has retinyl-palmitate lipase activity in human hepatic stellate cells. Human molecular genetics. 2014;23(15):4077-85.

54. Dongiovanni P, Petta S, Maglio C, Fracanzani AL, Pipitone R, Mozzi E, et al. Transmembrane 6 superfamily member 2 gene variant disentangles nonalcoholic steatohepatitis from cardiovascular disease. Hepatology (Baltimore, Md). 2015;61(2):506-14.

55. Goffredo M, Caprio S, Feldstein AE, D'Adamo E, Shaw MM, Pierpont B, et al. Role of TM6SF2 rs58542926 in the pathogenesis of nonalcoholic pediatric fatty liver disease: A multiethnic study. Hepatology (Baltimore, Md). 2016;63(1):117-25.

56. Beer NL, Tribble ND, McCulloch LJ, Roos C, Johnson PR, Orho-Melander M, et al. The P446L variant in GCKR associated with fasting plasma glucose and triglyceride levels exerts its effect through increased glucokinase activity in liver. Human molecular genetics. 2009;18(21):4081-8.

57. Santoro N, Caprio S, Pierpont B, Van Name M, Savoye M, Parks EJ. Hepatic De Novo Lipogenesis in Obese Youth Is Modulated by a Common Variant in the GCKR Gene. The Journal of clinical endocrinology and metabolism. 2015;100(8):E1125-32.

58. Valenti L, Alisi A, Nobili V. Unraveling the genetics of fatty liver in obese children: additive effect of P446L GCKR and I148M PNPLA3 polymorphisms. Hepatology (Baltimore, Md). 2012;55(3):661-3.

59. Stickel F, Moreno C, Hampe J, Morgan MY. The genetics of alcohol dependence and alcoholrelated liver disease. Journal of hepatology. 2017;66(1):195-211.

60. Mancina RM, Dongiovanni P, Petta S, Pingitore P, Meroni M, Rametta R, et al. The MBOAT7TMC4 Variant rs641738 Increases Risk of Nonalcoholic Fatty Liver Disease in Individuals of European Descent. Gastroenterology. 2016;150(5):1219-30.e6. 
61. Luukkonen PK, Zhou Y, Hyotylainen T, Leivonen M, Arola J, Orho-Melander M, et al. The MBOAT7 variant rs641738 alters hepatic phosphatidylinositols and increases severity of non-alcoholic fatty liver disease in humans. Journal of hepatology. 2016;65(6):1263-5.

62. Viitasalo A, Eloranta AM, Atalay M, Romeo S, Pihlajamaki J, Lakka TA. Association of MBOAT7 gene variant with plasma ALT levels in children: the PANIC study. Pediatric research. 2016;80(5):651-5.

63. Donati B, Dongiovanni P, Romeo S, Meroni M, McCain M, Miele L, et al. MBOAT7 rs641738 variant and hepatocellular carcinoma in non-cirrhotic individuals. Scientific reports. 2017;7(1):4492.

64. Thabet K, Chan HLY, Petta S, Mangia A, Berg T, Boonstra A, et al. The membrane-bound Oacyltransferase domain-containing 7 variant rs641738 increases inflammation and fibrosis in chronic hepatitis B. Hepatology (Baltimore, Md). 2017;65(6):1840-50.

65. Thabet K, Asimakopoulos A, Shojaei M, Romero-Gomez M, Mangia A, Irving WL, et al. MBOAT7 rs641738 increases risk of liver inflammation and transition to fibrosis in chronic hepatitis C. Nature communications. 2016;7:12757.

66. Lee HC, Inoue T, Imae R, Kono N, Shirae S, Matsuda S, et al. Caenorhabditis elegans mboa-7, a member of the MBOAT family, is required for selective incorporation of polyunsaturated fatty acids into phosphatidylinositol. Molecular biology of the cell. 2008;19(3):1174-84.

67. Lee HC, Inoue T, Sasaki J, Kubo T, Matsuda S, Nakasaki Y, et al. LPIAT1 regulates arachidonic acid content in phosphatidylinositol and is required for cortical lamination in mice. Molecular biology of the cell. 2012;23(24):4689-700.

68. Zarini S, Hankin JA, Murphy RC, Gijon MA. Lysophospholipid acyltransferases and eicosanoid biosynthesis in zebrafish myeloid cells. Prostaglandins \& other lipid mediators. 2014;113115:52-61. 
69. Gijon MA, Riekhof WR, Zarini S, Murphy RC, Voelker DR. Lysophospholipid acyltransferases and arachidonate recycling in human neutrophils. The Journal of biological chemistry. 2008;283(44):30235-45.

70. Di Paolo G, De Camilli P. Phosphoinositides in cell regulation and membrane dynamics. Nature. 2006;443(7112):651-7.

71. Sasaki T, Takasuga S, Sasaki J, Kofuji S, Eguchi S, Yamazaki M, et al. Mammalian phosphoinositide kinases and phosphatases. Progress in lipid research. 2009;48(6):307-43.

72. Backer JM. The regulation and function of Class III PI3Ks: novel roles for Vps34. The Biochemical journal. 2008;410(1):1-17.

73. Vogel P, Read RW, Hansen GM, Payne BJ, Small D, Sands AT, et al. Congenital hydrocephalus in genetically engineered mice. Veterinary pathology. 2012;49(1):166-81.

74. Ruis-Gonzalez MD, Canete MD, Gomez-Chaparro JL, Abril N, Canete R, Lopez-Barea J. Alterations of protein expression in serum of infants with intrauterine growth restriction and different gestational ages. Journal of proteomics. 2015;119:169-82.

75. Johansen A, Rosti RO, Musaev D, Sticca E, Harripaul R, Zaki M, et al. Mutations in MBOAT7, Encoding Lysophosphatidylinositol Acyltransferase I, Lead to Intellectual Disability Accompanied by Epilepsy and Autistic Features. American journal of human genetics. 2016;99(4):912-6.

76. Hayashi-Nishino M, Fujita N, Noda T, Yamaguchi A, Yoshimori T, Yamamoto A. A subdomain of the endoplasmic reticulum forms a cradle for autophagosome formation. Nature cell biology. 2009;11(12):1433-7.

77. de Brito OM, Scorrano L. An intimate liaison: spatial organization of the endoplasmic reticulum-mitochondria relationship. The EMBO journal. 2010;29(16):2715-23. 
78. Horl G, Wagner A, Cole LK, Malli R, Reicher H, Kotzbeck P, et al. Sequential synthesis and methylation of phosphatidylethanolamine promote lipid droplet biosynthesis and stability in tissue culture and in vivo. The Journal of biological chemistry. 2011;286(19):17338-50.

79. Hirata Y, Yamamori N, Kono N, Lee HC, Inoue T, Arai H. Identification of small subunit of serine palmitoyltransferase a as a lysophosphatidylinositol acyltransferase 1-interacting protein. Genes to cells : devoted to molecular \& cellular mechanisms. 2013;18(5):397-409.

80. Hashidate-Yoshida T, Harayama T, Hishikawa D, Morimoto R, Hamano F, Tokuoka SM, et al. Fatty acid remodeling by LPCAT3 enriches arachidonate in phospholipid membranes and regulates triglyceride transport. eLife. $2015 ; 4$.

81. Demeure O, Lecerf F, Duby C, Desert C, Ducheix S, Guillou H, et al. Regulation of LPCAT3 by LXR. Gene. 2011;470(1-2):7-11.

82. Kabir I, Li Z, Bui HH, Kuo MS, Gao G, Jiang XC. Small Intestine but Not Liver Lysophosphatidylcholine Acyltransferase 3 (Lpcat3) Deficiency Has a Dominant Effect on Plasma Lipid Metabolism. The Journal of biological chemistry. 2016;291(14):7651-60.

83. Rong X, Wang B, Dunham MM, Hedde PN, Wong JS, Gratton E, et al. Lpcat3-dependent production of arachidonoyl phospholipids is a key determinant of triglyceride secretion. eLife. 2015;4.

84. Cash JG, Hui DY. Liver-specific overexpression of LPCAT3 reduces postprandial hyperglycemia and improves lipoprotein metabolic profile in mice. Nutrition \& diabetes. 2016;6:e206.

85. Accili D, Drago J, Lee EJ, Johnson MD, Cool MH, Salvatore P, et al. Early neonatal death in mice homozygous for a null allele of the insulin receptor gene. Nature genetics. 1996;12(1):106-9.

86. Okamoto H, Nakae J, Kitamura T, Park BC, Dragatsis I, Accili D. Transgenic rescue of insulin receptor-deficient mice. The Journal of clinical investigation. 2004;114(2):214-23. 
87. Kleiner DE, Brunt EM, Van Natta M, Behling C, Contos MJ, Cummings OW, et al. Design and validation of a histological scoring system for nonalcoholic fatty liver disease. Hepatology (Baltimore, Md). 2005;41(6):1313-21.

88. Mederacke I, Dapito DH, Affo S, Uchinami H, Schwabe RF. High-yield and high-purity isolation of hepatic stellate cells from normal and fibrotic mouse livers. Nature protocols. 2015;10(2):305-15.

89. Milano M, Dongiovanni P, Artoni A, Gatti S, Rosso L, Colombo F, et al. Particulate matter phagocytosis induces tissue factor in differentiating macrophages. Journal of applied toxicology : JAT. 2016;36(1):151-60.

90. Nakae J, Park BC, Accili D. Insulin stimulates phosphorylation of the forkhead transcription factor FKHR on serine 253 through a Wortmannin-sensitive pathway. The Journal of biological chemistry. 1999;274(23):15982-5.

91. Nakae J, Kitamura T, Silver DL, Accili D. The forkhead transcription factor Foxo1 (Fkhr) confers insulin sensitivity onto glucose-6-phosphatase expression. The Journal of clinical investigation. 2001;108(9):1359-67.

92. Nakae J, Biggs WH, 3rd, Kitamura T, Cavenee WK, Wright CV, Arden KC, et al. Regulation of insulin action and pancreatic beta-cell function by mutated alleles of the gene encoding forkhead transcription factor Foxo1. Nature genetics. 2002;32(2):245-53.

93. Krawczyk M, Jimenez-Aguero R, Alustiza JM, Emparanza JI, Perugorria MJ, Bujanda L, et al. PNPLA3 p.I148M variant is associated with greater reduction of liver fat content after bariatric surgery. Surgery for obesity and related diseases : official journal of the American Society for Bariatric Surgery. 2016;12(10):1838-46. 
94. Rahdar M, McMahon MA, Prakash TP, Swayze EE, Bennett CF, Cleveland DW. Synthetic CRISPR RNA-Cas9-guided genome editing in human cells. Proceedings of the National Academy of Sciences of the United States of America. 2015;112(51):E7110-7. 